\title{
Guidelines for the physiotherapy management of the adult, medical, spontaneously breathing patient
}

\author{
J Bott, S Blumenthal, M Buxton, S Ellum, C Falconer, R Garrod, A Harvey, T Hughes, \\ M Lincoln, C Mikelsons, C Potter, J Pryor, L Rimington, F Sinfield, C Thompson, \\ P Vaughn, J White, on behalf of the British Thoracic Society Physiotherapy Guideline \\ Development Group
}

- Additional Appendices are published online at http://thorax. bmi.com/content/vol64/ issueSuppll and http://www. brit-thoracic.org.uk

Correspondence to: julia.bott@surreypct.nhs.uk

Received 19 November 2008 Accepted 15 February 2009

\section{GUIDELINE DEVELOPMENT GROUP}

\section{Steering group}

Julia Bott (Chair), support to Section 6, Consultant

Physiotherapist, Surrey PCT NW Locality

Sheric Ellum, support to Section 5, Consultant Physiotherapist, Guy's \& St Thomas' NHS Trust, London

Dr Rachel Garrod, support to Section 1, Reader, School of Physiotherapy, Faculty of Health and Social Care Sciences, Kingston University and St George's, University of London.

Dr Jennifer Pryor, support to Sections 3 and 4, Senior Research Fellow in Physiotherapy, Royal Brompton \& Harefield NHS Trust

Dr Lesley Rimington, support to Section 2, Lecturer School of Health and Rehabilitation, Keele University

\section{Section 1-COPD}

Sharon Baines, Clinical Specialist Physiotherapist, Chronic Lung Disease Service, NHS Central Lancashire

Amanda Dryer, Physiotherapy Clinical Lead in Respiratory Care, Central Manchester and Manchester Children's University Hospital

Robert Goddard, Superintendent Physiotherapist, County Durham and Darlington NHS Foundation Trust

Catherine Thompson, Senior Lecturer, York St John University

Dr John White (Chair), Respiratory Physician, York Hospitals NHS Trust

\section{Section 2-Asthma and disordered breathing}

Caroline Falconer (Co-Chair), Senior Physiotherapist, Papworth Hospital NHS Foundation Trust

Lianne Jongepier, Respiratory Specialist Physiotherapist, Service Lead COPD Team, Primary Care Centre, Colchester

Melanie Lincoln (Co-Chair), Team Leader Physiotherapist, Papworth Hospital NHS Foundation Trust

Christine Mikelsons, Consultant Respiratory Physiotherapist, Royal Free Hospital

Dr Mike Thomas, General Practitioner, Asthma UK Senior Research Fellow, University of Aberdeen

Jo Williams, Senior Pulmonary Rehabilitation Specialist Glenfield Hospital, University Hospitals of Leicester NHS Trust

\section{Section 3-Cystic fibrosis}

On behalf of the Association of Chartered Physiotherapists in Cystic Fibrosis

Penny Agent, Service Lead Physiotherapist, Royal Brompton \& Harefield NHS Trust

Gillian Davie, Senior I Physiotherapist, Cystic

Fibrosis Team, Aberdeen Royal Infirmary

Mary Dodd, Consultant Physiotherapist in

Cystic Fibrosis, University Hospital of South Manchester NHS Foundation Trust

Dr Sarah Elkin, Respiratory Physician, St Mary's Hospital, London

Tracey Hughes (Chair), Senior I Physiotherapist, Leeds Regional Adult Cystic Fibrosis Unit, Leeds

Teaching Hospitals NHS Trust

Margaret MacLeod, Senior I Physiotherapist, Cystic Fibrosis Team, Aberdeen Royal Infirmary

Nicola Mills, Senior I Physiotherapist, Adult Cystic Fibrosis Unit, University Hospitals of Leicester

\section{Section 4-Non-cystic fibrosis-related bronchiectasis}

Alex Harvey (Co-Chair), Lecturer in Physiotherapy, Brunel University

Fran Sinfield (Co-Chair), Superintendent Physiotherapist, Oxford Centre for Respiratory Medicine, The Churchill Hospital, Oxford

Dr Robert Wilson, Respiratory Physician, Royal Brompton \& Harefield NHS Trust

\section{Section 5-Non-obstructive/restrictive lung diseases}

Debbie Dykes, Clinical Specialist Respiratory Physiotherapy, St. Richards Hospital, Royal West Sussex NHS Trust, Chichester

Katie Ford, Team Lead, Respiratory Physiotherapy, Bristol Royal Infirmary, Bristol

Rachael Mitchell, Specialist Respiratory Physiotherapist, Luton and Dunstable Hospital NHS Foundation Trust

Catherine Potter (Chair), Specialist Respiratory Physiotherapist, The Whittington Hospital NHS Trust, London

Fiona Rushmer, Physiotherapy Manager, Ashtead Hospital, Surrey

Dr Paul Tate, Respiratory Physician, St. Richards Hospital, Royal West Sussex NHS Trust, Chichester

Jennifer Tomkinson, Respiratory Specialist Physiotherapist, Bristol Primary Care Trust 


\section{Section 6-Neuromuscular diseases and chest wall disorders}

Dr Steve Banham, Respiratory Physician, Glasgow Royal Infirmary

Sharron Blumenthal (Co-Chair), Lecturer in Physiotherapy, Glasgow Caledonian University

Caroline Brown, Principal Physiotherapist, Hospital of North Staffordshire NHS Trust

Rebekah Hooker, Advanced Physiotherapist, University Hospital of North Staffordshire NHS Trust

Lisa Morrison, Clinical Specialist Physiotherapist, Gartnaval Hospital, Glasgow

Pamela Vaughn (Co-Chair), Clinical Specialist Physiotherapist, Stobhill Hospital, Glasgow

Nicola Williams, Specialist Physiotherapist, Blackpool, Fylde and Wyre Hospitals NHS Trust.

\section{Section 7-Workforce issues}

Maria Buxton, Consultant Physiotherapist, Central Middlesex Hospital and Brent PCT

Christine Mikelsons, Consultant Respiratory Physiotherapist, Royal Free Hospital

\section{SUMMARY OF RECOMMENDATIONS Introduction}

Physiotherapy should be offered to patients with a variety of medical respiratory conditions, with the aim of breathlessness management and symptom control, mobility and function improvement or maintenance, and airway clearance and cough enhancement or support. Strategies and techniques include: rehabilitation, exercise testing (including for ambulatory oxygen assessment), exercise prescription, airway clearance, and positioning and breathing techniques. Physiotherapy may be helpful for postural and/or musculoskeletal dysfunction and pain, and provide help in improving continence, especially during coughing and forced expiratory manoeuvres. Physiotherapists are usually central to the delivery of pulmonary rehabilitation and may be instrumental in the non-invasive ventilation service. Physiotherapists are frequently involved in the delivery of oxygen and some nebulised substances, as well as providing vital monitoring of, for example, ventilatory function and cough effectiveness. Some complementary therapies may be appropriate in some situations (Web Appendix 1).

Recommendations are listed for each diagnostic group. A concise version of this guideline is available on the BTS website.

\section{Chronic obstructive pulmonary disease (COPD) \\ Management of breathlessness \\ Positioning \\ Recommendations}

- Advise on passively fixing the shoulder girdle for optimising ventilatory muscle efficiency and relief of breathlessness. (Grade D)

- Assess the effectiveness of forward lean sitting on relief of breathlessness in all patients with COPD, in both the chronic and acute settings. (Grade $\mathrm{C}$ )

- Advise modification of the forward lean position for use in standing and lying, for patients for whom forward lean sitting is effective. (Grade D)

\section{Walking aids}

Recommendations

- Assess the effectiveness of a rollator frame for patients with COPD disabled by breathlessness. (Grade B)
- Assess the effectiveness of a gutter rollator frame in the acute setting, for patients with COPD severely disabled by breathlessness, especially the elderly. (Grade B)

- Teach individualised energy conservation techniques to help reduce dyspnoea during activities of daily living. (Grade D)

\section{Breathing techniques}

Recommendations

- Teach patients with COPD breathing control at rest to see if it helps relieve dyspnoea. (Grade D)

- Diaphragmatic breathing should not be taught routinely to patients with severe COPD. (Grade C)

- Teach pursed lips breathing during exertion as a potential strategy to reduce respiratory rate and aid recovery in patients with COPD. (Grade C)

- Teach exhalation on effort ("blow as you go!") as a potential strategy to reduce dyspnoea in patients with COPD. (Grade D)

- Teach relaxed, slower, deeper breathing as a potential strategy to facilitate more effective ventilation during exertion in patients with COPD. (Grade D)

- Teach paced breathing as a strategy to maintain control of breathing and reduce dyspnoea during exertion in patients with COPD. (Grade D)

\section{Ventilation feedback training}

Recommendation

- Ventilation feedback training is not indicated in patients with COPD. (Grade C)

\section{Managing anxiety and panic}

Recommendation

- Teach patients with COPD positioning, breathing and relaxation strategies to help manage anxiety and panic attacks. (Grade D)

\section{Pulmonary rehabilitation}

Recommendations

- Pulmonary rehabilitation should include exercise training of the muscles of ambulation. (Grade A)

- Pulmonary rehabilitation should incorporate strength training of both upper and lower limbs. (Grade A)

- Information, advice and education should be integral to pulmonary rehabilitation. (Grade A)

- Pulmonary rehabilitation should be made available to all appropriate patients with COPD. (Grade A)

\section{Field exercise tests}

Recommendation

- The recommended number of practice walks must be included when assessing exercise tolerance with a field exercise test for the prescription of either exercise or ambulatory oxygen. (Grade C)

\section{Peri- and postexacerbation pulmonary rehabilitation}

Recommendations

- Consider pulmonary rehabilitation soon after exacerbation for patients with COPD. (Grade B)

- Consider some form of rehabilitation during exacerbation to maintain mobility and function in patients with COPD. (Grade D) 


\section{Respiratory muscle training}

Recommendations

- Consider adding inspiratory muscle training to a general exercise programme where respiratory muscle weakness is thought to be contributing to the patient's problems. (Grade A)

- Consider inspiratory muscle training in the management of COPD to improve respiratory muscle strength and/or endurance. (Grade A)

- Consideration of maintenance of an inspiratory muscle training programme is required. (Grade D)

- Devices that incorporate control of breathing pattern and flow rate should be considered over devices that do not have this function. (Grade D)

\section{Non-invasive ventilation}

Recommendations

- Non-invasive ventilation should be offered to patients with COPD and acute hypercapnic respiratory failure, if they meet recommended BTS criteria. (Grade A)

- Facilities for non-invasive ventilation should be available $24 \mathrm{~h}$ per day in all hospitals likely to admit such patients. (Grade A)

\section{Intermittent positive pressure breathing}

Recommendations

- Tidal volume must be increased to achieve a therapeutic effect. (Grade C)

- Care must be taken to ensure settings achieve patient synchrony with the device to reduce work of breathing. (Grade C)

- Short periods of daytime intermittent positive pressure breathing should not be used to treat chronic respiratory failure in stable COPD. (Grade A)

- Consider intermittent positive pressure breathing in acute exacerbations of COPD where patients present with retained secretions but are too weak or tired to generate an effective cough. (Grade D)

- When using intermittent positive pressure breathing in acute respiratory failure, an $\mathrm{FiO}_{2}$ of 0.4 may be used. (Grade B)

\section{Oxygen therapy}

Recommendations

- Administer oxygen therapy, in both the acute and domiciliary settings, according to current national guidance. (Grade A)

- Consider assessing the benefit of a walking aid to transport the ambulatory oxygen, especially for the more disabled patient. (Grade B)

\section{Airway clearance techniques}

Recommendations

- Consider the active cycle of breathing techniques (which includes the forced expiration technique), autogenic drainage and plain or oscillating positive expiratory pressure for patients with stable COPD who need an airway clearance technique to assist in the removal of secretions. (Grade C)

- Incorporate postural drainage only if it further aids clearance and has no detrimental effects. (Grade D)

\section{Pelvic floor muscle training}

Recommendations
- Patients with COPD should be questioned about their continence status. (Grade D)

- All patients with chronic cough, irrespective of continence status, should be taught to contract the pelvic floor muscles before forced expirations and coughing ("The Knack"). (Grade D)

- If problems of leakage are identified, patients should be referred to a physiotherapist specialising in continence. (Grade D)

\section{Asthma and disordered breathing \\ Asthma \\ Breathing exercises}

Recommendations

- Breathing exercises, incorporating reducing respiratory rate and/or tidal volume and relaxation training, should be offered to patients to help control the symptoms of asthma and improve quality of life. (Grade A)

- The Buteyko breathing technique may be considered to help patients to control the symptoms of asthma. (Grade B)

- The use of suitable tools such as an asthma-specific quality of life measure, measures of anxiety and depression and the Nijmegen Questionnaire should be used to establish baseline severity of symptoms and monitor progress with treatment. (Grade B)

\section{Exercise training}

Recommendations

- Physical training should be advised for improvements in fitness and cardiorespiratory performance in patients with asthma. (Grade B)

- Physical training should be advised to help reduce breathlessness and improve health-related quality of life in people with asthma. (Grade B)

- Physical training programmes should aim to reach a minimum of activity as per the American College of Sports Medicine guidelines. (Grade A)

\section{Disordered breathing (hyperventilation syndrome) Hyperventilation syndrome}

Recommendation

- Breathing retraining incorporating reducing respiratory rate and/or tidal volume should be offered as a first-line treatment for hyperventilation syndrome, with or without concurrent asthma. (Grade B)

\section{Cystic fibrosis}

\section{Exercise}

Recommendations

- Exercise should be an integral part of the management of patients with cystic fibrosis. (Grade B)

- Physical training programmes should aim to reach a minimum of activity as per the American College of Sports Medicine guidelines. (Grade A)

\section{Airway clearance}

Recommendations

- Teach patients with cystic fibrosis an airway clearance technique to increase mucus transport in the short term. (Grade A)

- Self-administered techniques should be the first-line airway clearance techniques offered in order to improve adherence to treatment. (Grade B) 
- Patient preference for techniques should be considered in order to improve adherence to treatment. (Grade B)

\section{Postural drainage and manual techniques}

Recommendations

- Individually assess the effect and acceptability of gravityassisted positioning in patients with cystic fibrosis. (Grade B)

- Individually assess the effect and acceptability of modified gravity-assisted positioning in individual patients with cystic fibrosis. (Grade C)

- If patients using independent techniques are unable to clear secretions effectively, chest wall vibration should be considered. (Grade C)

\section{Simple airway clearance techniques}

Recommendations

- Consider the active cycle of breathing techniques when recommending an airway clearance technique for adults with cystic fibrosis. (Grade A)

- Consider autogenic drainage when recommending an airway clearance technique for adults with cystic fibrosis. (Grade A)

- Consider positive expiratory pressure when recommending an airway clearance technique for adults with cystic fibrosis. (Grade A)

- Consider oscillating positive expiratory pressure devices when recommending an airway clearance technique for adults with cystic fibrosis. (Grade A)

- Exercise in isolation should not be used as an airway clearance technique for patients with cystic fibrosis unless adherence to other techniques is problematic. (Grade D)

- The addition of exercise to an appropriate physiotherapy regimen should be considered to increase airway clearance further. (Grade D)

\section{Mechanical devices for airway clearance}

Recommendations

- Consider high-frequency chest wall compression/oscillation when recommending an airway clearance technique for adults with cystic fibrosis. (Grade A)

- High-frequency chest wall oscillation is not recommended during an infective exacerbation. (Grade B)

- Consider mechanical vibration when recommending an airway clearance technique for adults with cystic fibrosis. (Grade A)

- Consider intrapulmonary percussive ventilation when recommending an airway clearance technique for adults with mild to moderate cystic fibrosis. (Grade A)

\section{Non-invasive ventilation and intermittent positive pressure breathing}

Recommendations

- Try non-invasive ventilation for airway clearance where there is evidence of respiratory muscle weakness or fatigue. (Grade A)

- Try non-invasive ventilation where desaturation is present during airway clearance. (Grade A)

- Try non-invasive ventilation when the patient has difficulty clearing bronchial secretions with other techniques. (Grade A)

- Consider a trial of intermittent positive pressure breathing for airway clearance as an alternative to non-invasive ventilation, where the indications for non-invasive ventilation in this situation exist. (Grade D)

\section{Suction}

Recommendation

- Suction should not be considered for use as a routine airway clearance technique in non-intubated patients with cystic fibrosis. (Grade D)

\section{Inhalation therapies}

\section{Oxygen therapy and humidification}

Recommendations

- Administer oxygen therapy, in both the acute and domiciliary settings, according to current national guidance. (Grade A)

- Assess patients with advanced disease for supplemental ambulatory oxygen therapy. (Grade D)

- Bubble-through humidification should be avoided due to no evidence of clinical benefit and increased infection risk (Grade A)

\section{Hypertonic saline}

Recommendations

- Consider the addition of hypertonic saline when enhancing the effectiveness of an airway clearance technique. (Grade A)

- A predose bronchodilator should be used to minimise bronchospasm with inhalation of hypertonic saline. (Grade D)

- A bronchoconstriction trial should be carried out at the initial dose of hypertonic saline to ensure safety and suitability for the patient. (Grade D)

\section{RhDNase for physiotherapy}

Recommendations

- RhDNase should be prescribed as per national and local guidelines. (Grade A)

- Consider the use of inhaled RhDNase for enhancing airway clearance effectiveness. (Grade D)

- Consider inhalation therapy with RhDNase for increasing exercise capacity. (Grade D)

\section{Thoracic mobility and strengthening}

Recommendations

- Question patients with cystic fibrosis about musculoskeletal problems and back pain. (Grade D)

- Assess the problem if present and institute appropriate posture correction, chest wall mobility and stretching exercises or manual therapy treatments where indicated. (Grade D)

\section{Pelvic floor muscle training}

Recommendations

- Question patients with cystic fibrosis about their continence status. (Grade D)

- All patients with cystic fibrosis, irrespective of continence status, should be taught to contract the pelvic floor muscles before forced expirations and coughing ("The Knack"). (Grade D)

- If problems of leakage are identified, patients should be referred to a physiotherapist specialising in continence. (Grade D) 
- Therapeutic interventions should include an element of endurance training of the pelvic floor muscles to meet the demands of prolonged coughing. (Grade D)

\section{Infection control}

Recommendation

- Physiotherapists caring for patients with cystic fibrosis should be aware of consensus documents regarding infection control. (Grade C)

\section{Non-cystic fibrosis-related bronchiectasis Pulmonary rehabilitation}

Recommendations

- Offer pulmonary rehabilitation to individuals with noncystic fibrosis-related bronchiectasis with breathlessness affecting activities of daily living. (Grade $\mathrm{A}$ )

- Consider the use of inspiratory muscle training in conjunction with conventional pulmonary rehabilitation to enhance the maintenance of the training effect. (Grade B)

\section{Airway clearance techniques}

Recommendations

- Teach all patients with bronchiectasis and a chronic, productive cough, and/or evidence of mucus plugging on $\mathrm{CT}$, an airway clearance technique for use as necessary. (Grade D)

- Review the effectiveness and acceptability of the chosen airway clearance technique within approximately 3 months of the initial visit. (Grade D)

- Patients should be made aware of other available airway clearance technique options. (Grade D)

\section{Postural drainage}

Recommendations

- Where it is found to enhance airway clearance and has no unwanted side effects, postural drainage should be taught and encouraged. (Grade B)

- Patient preference and adherence to treatment must be taken into account. (Grade B)

- Take comorbidities, and contraindications and precautions to head-down tilt positions into account. (Grade D)

- Consider offsetting the increased load of breathing by the use of non-invasive ventilation or intermittent positive pressure breathing where postural drainage is essential for clearing secretions in a breathless patient. (Grade D)

\section{Simple airway clearance techniques}

\section{Recommendations}

- Consider the active cycle of breathing techniques when recommending an airway clearance technique for adults with non-cystic fibrosis-related bronchiectasis. (Grade A)

- Consider oscillating positive expiratory pressure when recommending an airway clearance technique for adults with non-cystic fibrosis-related bronchiectasis. (Grade A)

- The test of incremental respiratory endurance should not be considered as a first-line airway clearance technique. (Grade B)

- The inclusion of postural drainage should be considered for all airway clearance techniques. (Grade B)

- The inclusion of the forced expiration technique should be considered for all airway clearance techniques. (Grade B)

Adjuncts to enhance airway clearance

Recommendations
- Consider nebulised sterile water inhalation before treatment to enhance sputum clearance. (Grade B)

- Consider nebulised $\beta_{2}$-agonists before treatment to enhance sputum clearance. (Grade B)

- Consider nebulised hypertonic saline before airway clearance to increase sputum yield, reduce sputum viscosity and improve ease of expectoration. (Grade B)

- When first administered, $\mathrm{FEV}_{1}$ or peak expiratory flow rate should be measured before and after nebulised hypertonic saline to assess for possible bronchoconstriction. (Grade D)

- Pretreat with a bronchodilator, particularly for those with bronchial hyper-reactivity. (Grade D)

- Consider nebulised normal saline before airway clearance to increase sputum yield, reduce sputum viscosity and improve ease of expectoration when hypertonic saline is not suitable or available. (Grade B)

\section{Non-invasive ventilation and intermittent positive pressure breathing} Recommendation

- Consider non-invasive ventilation or intermittent positive pressure breathing to augment tidal volume and reduce the work of breathing in patients who are becoming fatigued and finding airway clearance difficult. (Grade D)

\section{Pelvic floor muscle training}

Recommendations

- Patients should be questioned about their continence status. (Grade D)

- All patients with chronic cough, irrespective of continence status, should be taught to contract the pelvic floor muscles before forced expirations and coughing ("The Knack"). (Grade D)

- If problems of leakage are identified, patients should be referred to a physiotherapist specialising in continence. (Grade D)

\section{Restrictive lung conditions}

There is a paucity of evidence on physiotherapy for these conditions.

\section{Lung fibrosis}

\section{Pulmonary rehabilitation}

Recommendation

- All patients with chronic restrictive conditions, such as pulmonary fibrosis, should be considered for pulmonary rehabilitation. (Grade B)

\section{Community-acquired pneumonia}

Recommendations

- Medical condition permitting, patients admitted to hospital with uncomplicated community-acquired pneumonia should sit out of bed for at least 20 min within the first $24 \mathrm{~h}$ and increase mobility each subsequent day of hospitalisation. (Grade B)

- Patients admitted with primary uncomplicated pneumonia should not be treated with traditional airway clearance techniques routinely. (Grade B)

- In patients with uncomplicated community-acquired pneumonia admitted to hospital, the regular use of positive expiratory pressure should be considered. (Grade B)

- Continuous positive airway pressure should be considered for patients with pneumonia and type I respiratory failure 
who remain hypoxaemic despite optimum medical therapy and oxygen. (Grade C)

- Non-invasive ventilation can be considered for selected patients with community-acquired pneumonia and type II respiratory failure, especially those with underlying COPD. (Grade C)

- Patients admitted with primary uncomplicated pneumonia should not be treated with traditional airway clearance techniques and intermittent positive pressure breathing in combination. (Grade B)

\section{Neuromuscular diseases and musculoskeletal disorders of the chest wall}

Chest wall disorders

Pulmonary rehabilitation and ambulatory oxygen

Recommendations

- Offer patients with chest wall restriction post-tuberculosis pulmonary rehabilitation. (Grade B)

- Offer patients with chest wall deformity from other causes, who have reduced exercise capacity and/or breathlessness on exertion, pulmonary rehabilitation. (Grade C)

- Assess patients with moderate to severe kyphoscoliosis who desaturate on exercise for ambulatory oxygen. (Grade D)

\section{Respiratory muscle training and breathing exercises}

Recommendation

- Consider respiratory muscle training in patients with kyphoscoliosis. (Grade D)

\section{Spinal cord injuries}

\section{Monitoring}

Recommendations

- Monitor the patient with spinal cord injury for the signs and symptoms of respiratory problems and take appropriate action if abnormal or changing. (Grade A)

- Measure vital capacity routinely in the patient with upper spinal cord injury and take appropriate action if falling. (Grade D)

- Alert medical staff if vital capacity falls to 1 litre or less. (Grade D)

\section{Positioning}

Recommendations

- Consider the supine position to maximise vital capacity. (Grade B)

- Assess the head-up $30^{\circ}$ position for improving pulmonary function. (Grade C)

- The head-down position should only be used where there is a demonstrable need and only with extreme caution. (Grade D)

- Any patient, especially those with early spinal cord injury, should be carefully monitored for signs of hypoxaemia in head-down positions. (Grade D)

- Take comorbidities and contraindications and precautions to head-down tilt positions into account. (Grade D)

\section{Abdominal binders}

Recommendations

- Assess the effect of an abdominal binder for upright sitting where improvement in either vital capacity or respiratory muscle function is required. (Grade D)

- Patients using non-elastic binders should be monitored closely. (Grade D)
- When using an abdominal binder, the optimal position for the individual patient should be determined. (Grade D)

\section{Management of cough and airway secretions}

\section{Assisted coughing}

Recommendations

- Try manually assisted coughing for patients with an ineffective cough. (Grade D)

- The upright seated position should be considered initially. (Grade D)

- The abdominal thrust (Heimlich-style manoeuvre) should be considered initially. (Grade D)

\section{Mechanical insufflation-exsufflation}

Recommendations

- Mechanical insufflation-exsufflation should be considered for individuals with upper spinal cord injury, if simpler techniques fail to produce an adequate effect. (Grade D)

- Where cough effectiveness remains inadequate with mechanical insufflation-exsufflation alone, combine it with manually assisted coughing. (Grade D)

\section{Functional electrical stimulation}

Recommendation

- Consider electrical stimulation of the abdominal muscles as a possible means of enhancing lung volumes and cough effectiveness. (Grade C)

\section{Exercise}

Recommendation

- Active exercise should be encouraged in patients confined to a wheelchair as a result of spinal cord injury. (Grade D)

\section{Breathing exercises}

Recommendation

- Deep breathing exercises should be encouraged in patients with spinal cord injury. (Grade D)

\section{Respiratory muscle training}

Recommendations

- Inspiratory muscle training may be considered for patients with upper spinal cord injury to improve respiratory muscle strength. (Grade C)

- Inspiratory muscle training may be considered for patients with upper spinal cord injury to improve vital capacity and residual volume. (Grade $\mathrm{C}$ )

- Training of the accessory muscles of respiration with progressive loading should be considered. (Grade D)

\section{Neuromuscular disease}

\section{Oxygen therapy and non-invasive ventilation}

Recommendations

- Low-flow (high $\mathrm{FiO}_{2}$ ) oxygen therapy should be avoided or used with extreme caution due to the risk of carbon dioxide retention in patients with neuromuscular disease. (Grade A)

- Consider non-invasive ventilation as an initial intervention in patients with, or at risk of developing, hypercapnia. (Grade D) 
Peak cough flow (PCF) monitoring

Recommendations

- Peak cough flow should be measured regularly in patients with neuromuscular disease. (Grade D)

- Measure peak cough flow additionally at the time of an acute respiratory tract infection. (Grade D)

- When peak cough flow is equal to or less than $270 \mathrm{l} / \mathrm{min}$ in a medically stable patient, introduce strategies for assisted airway clearance to raise it above $270 \mathrm{l} / \mathrm{min}$. (Grade D)

- When peak cough flow is equal to or less than $160 \mathrm{l} / \mathrm{min}$, additional strategies to assist secretion clearance must be used. (Grade D)

- If peak cough flow remains equal to or less than $160 \mathrm{l} / \mathrm{min}$ despite additional strategies, contact medical colleagues to discuss ventilation and/or airway management needs. (Grade D)

\section{Airway clearance techniques}

\section{Maximal insufflation capacity}

Recommendations

- When oxygen saturation falls below 95\% the use of noninvasive ventilation and/or strategies to aid airway clearance should be considered. (Grade D)

- Use some form of maximal insufflation strategy to improve effective cough generation when vital capacity falls below $1500 \mathrm{ml}$ or $50 \%$ predicted. (Grade D)

- Use single maximal insufflation techniques for patients with bulbar dysfunction who are unable to breath stack. (Grade D)

- Teach patients without bulbar muscle involvement unaided breath stacking to improve cough effectiveness independently where possible. (Grade D)

- Regular breath stacking (10-15 times three times per day) to maximal insufflation capacity should be performed by patients with vital capacity of less than $2000 \mathrm{ml}$ or $50 \%$ predicted. (Grade D)

\section{Glossopharyngeal breathing}

Recommendations

- Consider teaching glossopharyngeal breathing to patients with reduced vital capacity to maintain range of chest wall movement and pulmonary compliance. (Grade D)

- Consider teaching glossopharyngeal breathing as one of the means of achieving maximal insufflation capacity in patients who have difficulty in clearing secretions. (Grade D)

- Consider teaching glossopharyngeal breathing to ventilatordependent patients to allow some ventilator-free breathing time. (Grade D)

- Consider teaching glossopharyngeal breathing to patients with decreased voice strength. (Grade D)

\section{Manually assisted coughing}

Recommendations

- Manually assisted coughing should be used to increase peak cough flow in patients with neuromuscular disease. (Grade C)

- Combine manually assisted coughing with a maximal insufflation capacity strategy. (Grade D)

- Abdominal thrusts should be performed standing in front of the patient where possible to assist communication. (Grade D)

\section{Mechanical insufflation-exsufflation}

Recommendations

- Consider mechanical insufflation-exsufflation as a treatment option in patients with bulbar muscle involvement who are unable to breath stack. (Grade D)

- Consider mechanical insufflation-exsufflation for any patient who remains unable to increase peak cough flow to effective levels with other strategies. (Grade D)

- Where cough effectiveness remains inadequate with mechanical insufflation-exsufflation alone, combine it with manually assisted coughing. (Grade D)

\section{Intrapulmonary percussive ventilation}

Recommendations

- Intrapulmonary percussive ventilation may be considered for patients with neuromuscular disease to aid loosening of secretions prior to removal where there is evidence of sputum retention and other techniques have failed. (Grade D)

- In patients with ineffective cough, assisted cough strategies must be used additionally to increase cough effectiveness. (Grade D)

- Patients using intrapulmonary percussive ventilation must be monitored closely during and after treatment for any adverse response. (Grade D)

\section{Conclusion}

This is the first extensive systematic literature review undertaken of the existing evidence surrounding comprehensive physiotherapy management of the spontaneously breathing medical respiratory adult patient and providing graded recommendations for practise.

\section{INTRODUCTION AND BACKGROUND TO THE GUIDELINES}

\section{Purpose of the guidelines}

This document has arisen as a result of the need for clarity concerning physiotherapy techniques and the evidence supporting them. It is a collaborative work between the British Thoracic Society (BTS) and the Association of Chartered Physiotherapists in Respiratory Care (ACPRC), the respiratory clinical interest group of the Chartered Society of Physiotherapy (CSP), now in existence for 25 years. Its purpose is critically to appraise the evidence for respiratory physiotherapy techniques, formulating evidence-based recommendations where possible. The guidelines are to inform all respiratory physicians and physiotherapists working in respiratory care of the scope of physiotherapy practice and the current evidence supporting the use of physiotherapeutic techniques. These guidelines cover only physiotherapy management of adult patients with medical, rather than surgically related, respiratory problems. They also exclude the management of physiotherapy for the critically ill patient requiring invasive ventilatory support. It is envisaged that further guidelines will ensue covering these topics at later dates.

\section{A brief history of respiratory physiotherapy and its relationship to the BTS}

The CSP describes physiotherapy in the following way "physiotherapy encompasses a range of interventions, services and advice aimed at restoring, maintaining and improving people's function and movement and thereby maximising the quality of their lives". ${ }^{1}$ Nurses trained in massage can be said to have founded physiotherapy as a profession in 1894. This small band of nurses formed their society as a means of protecting 
their newfound skills from misrepresentation; massage in Victorian society carried with it much the same connotation as in society today. The Society of Trained Masseuses was incorporated by Royal Charter in 1920, and by 1944 had become the CSP, the second oldest national physiotherapy organisation. ${ }^{2}$ Some of the earliest reports of treatment evaluation were in $1901^{3}$ and $1915,{ }^{4}$ describing physiotherapy techniques that we would still recognise today. Ewart ${ }^{3}$ described a method of postural drainage for the removal of secretions, while MacMahon ${ }^{4}$ described the use of breathing exercises combined with physical exercise for chest injuries received during the First World War.

Respiratory physiotherapy continued to grow under the auspices of the medical profession until 1977 when the Department of Health instituted professional autonomy for physiotherapists. The CSP followed this in 1978 by changing their statutes to allow physiotherapists to treat patients without medical referral; physiotherapists are therefore independent practitioners. ${ }^{2}$ To this day, respiratory physiotherapy techniques continue to be described and evaluated in the medical literature. Despite this, physiotherapy is not always considered an essential component of a respiratory service. In recent years, the BTS has sought to help redress this, and worked closely with the ACPRC to include physiotherapy in all its initiatives.

Physiotherapists are aware of the lack of evidence surrounding many techniques regularly employed today and are striving to establish good quality research. Throughout the last century, a number of centres of excellence for respiratory physiotherapy have arisen. These centres, and many individual physiotherapists, have strived rigorously to evaluate and to report treatment techniques. Opportunities for physiotherapists to research physiotherapy techniques have been limited due to lack of research funding and expertise. With the onset of an allgraduate profession in 1992, research in respiratory physiotherapy has increased. Many members of the BTS and its Council have supported this desire for better levels of evidence upon which current physiotherapy practice can operate, and the culmination of this support and collaboration is the creation of these guidelines.

It is hoped that this document will encourage the referral for physiotherapy, rather than for a specific technique, since the appropriate treatment may be a combination of techniques, specifically tailored to the individual based on their symptom(s) and problem(s), not their diagnosis.

\section{GUIDELINE DEVELOPMENT}

A steering group of five expert physiotherapists, including the BTS Standards of Care Committee (SOCC) and BTS Council physiotherapy representatives, and the chair of the ACPRC, was formed in October 2004 to define the method, format and scope of the guidelines. Although physiotherapists treat patients by physiological or physical problem, irrespective of diagnosis, a consensus was reached by the steering group and the SOCC to formulate the guidelines in the usual medical format-that is, by diagnosis. It was agreed to study the physiotherapy evidence in the following diagnostic groups:

1. Chronic obstructive pulmonary disease (COPD)

2. Asthma and disordered breathing

3. Cystic fibrosis (CF)

4. Non-cystic fibrosis-related bronchiectasis

5. Restrictive lung diseases, for example pneumonia and interstitial fibrosis
6. Pulmonary disorders arising from chest wall and neuromuscular conditions.

A seventh section was added to attempt to address the recommendations for the physiotherapy workforce required to deliver the treatments recommended in these guidelines. Some preliminary guidance is given on expected treatment times for most interventions, but further detailed work by the BTS and the ACPRC on workforce requirements will follow.

A national call via the ACPRC newsletter and interactive CSP website was then made in early 2005 to find contributors. As a result, 32 physiotherapists volunteered, including individuals representing the North Yorkshire Group and the Association of Chartered Physiotherapists in Cystic Fibrosis (ACPCF). Six working parties, one for each diagnostic group, were created. The working parties were formed, for ease of meetings and workload sharing, with the exception of group 3 (Cystic fibrosis), by geographical location of volunteers, and consisted of several physiotherapists supported by a member of the steering group. A physician with expertise in each of the six diagnostic categories was invited to provide medical support to every group. Patient representatives and/or a relevant patient body, for example Asthma UK, were invited to comment on each section. The guideline development group commenced work in April 2005 and the BTS guidelines development checklist was adhered to at all stages.

A comprehensive list of search terms encompassing specific physiotherapy techniques and diagnostic terms was compiled, and the search conducted by the Centre for Research and Dissemination (CRD), York, UK. The searching was undertaken in two stages. The following databases were searched in May 2005: Medline (2003-2005/03) (Silver platter on CD), Cochrane Database of Systematic Reviews (Cochrane Library 2005, issue 2), Database of Abstracts of Reviews of Effects (DARE; the CRD administration database), National Research Register (2005, issue 2), Clinical evidence, National Guideline Clearinghouse, National Institute for Health and Clinical Excellence (NICE), National Electronic Library for Health Guidelines Finder, Physiotherapy Evidence Database (PEDro) and Turning Research into Practice Database. Following these searches, additional focused searches were undertaken in the six diagnostic groups in the following databases: Medline (Ovid interface) 1966-2005/11; Cinahl (Ovid interface) 1982-2005/11; Cochrane Database of Systematic Reviews and the Central Register of Controlled Trials (Cochrane Library 2005 issue 4); DARE was searched on the CRD in-house system up to 11 January 2006.

A total of 7856 titles were identified and screened. Exclusion criteria were: non-English language, opinion reviews, paediatric only or animal subjects, studies not including relevant physiotherapy management or not directly relevant to physiotherapy, and studies evaluating outcome measures or new tools. Further screening of abstracts revealed 316 papers suitable for inclusion. Targeted hand-searching was performed when obvious omissions from the search were identified. Recent relevant articles have been included, as well as grey literature where appropriate. Each included article was read and appraised for methodological rigour, to the Scottish Intercollegiate Guidelines Network (SIGN) ${ }^{5}$ criteria, by two individuals, with a third in the event of a disagreement. Evidence tables were created and a typical example is given on the BTS website (Web Appendix 2). Levels of evidence were assigned to each paper and ensuing recommendations graded accordingly (table 1). Good practice points have been formulated where there is no, nor likely to be, published evidence, but which represent best 
practice, deemed "clinical common sense", ${ }^{5}$ based on the expert opinion of the guidelines development group and/or the patient representative(s). A glossary of physiotherapy terms is given in Appendix A.

A brief overview of possible complementary therapies used or recommended by physiotherapists, but focusing primarily on those used in asthma, is included in Web Appendix 1. To accompany Section $6 c$, neuromuscular disease, a management algorithm for physiotherapists has been compiled (Appendix B) and instructions for performing peak cough flow measurements are provided (Appendix C).

Patient information leaflets (downloadable from the BTS website) have been created for each diagnosis (Web Appendices 3-9), based on the evidence for physiotherapeutic techniques. The exceptions are: cystic fibrosis (CF), since comprehensive leaflets on physiotherapy treatment are available via the CF Trust http://www.cftrust.org.uk/aboutcf/publications/factsheets, and for restrictive lung disorders, since there was insufficient evidence to warrant it. A downloadable action plan for patients with neuromuscular disease has also been provided (Web Appendix 10). Finally, a list of commonly used devices with company contact details has been compiled in Web Appendix 11. A concise version of this guideline is also available on the BTS website.

\section{SECTION 1 CHRONIC OBSTRUCTIVE PULMONARY DISEASE (COPD)}

\section{Introduction}

COPD is a progressive, irreversible, respiratory disease affecting approximately 600000 UK adults. ${ }^{6}$ Morbidity and mortality are high, and COPD leads to significant disability and distressing symptoms. A number of important guidelines have been published concerning the management of COPD; in 1997 the BTS published the first British guidance, ${ }^{6}$ and more recently the NICE published comprehensive evidence-based clinical guidelines. ${ }^{7}$ Internationally there have been publications as far back as $1987^{\circ}$ and as recently as $2004 .{ }^{9}$ Few of these guidelines, however, relate specifically to the physiotherapeutic management of COPD, and then only sparsely. In 2000, researchers from The Netherlands published a systematic review of evidence for physiotherapy in $\mathrm{COPD}^{10}$ and, whilst this guidance is of relevance to the UK, differences in delivery of care and the unique organisation of the British National Health Service (NHS) mean that implementation has been poor.

Physiotherapists have been instrumental in the management of COPD for decades ${ }^{11-13}$; they play an important role in the assessment and non-pharmacological treatment of breathing dysfunction and dyspnoea, ${ }^{10}$ in the assessment for and the delivery of pulmonary rehabilitation $(\mathrm{PR})^{14}$ and non-invasive ventilation (NIV), ${ }^{15-20}$ and in the management of impaired airway clearance. ${ }^{21}$ Furthermore, self-management and patient education are recognised as important, cost-effective components of long-term care and are frequently delivered by physiotherapists. ${ }^{22}$

\section{Breathlessness management \\ Positioning}

Many patients with COPD adopt a rapid, shallow breathing pattern, frequently with chest wall and abdominal asynchrony. In patients with hyperinflated lungs and an increased expiratory reserve volume (ERV), the inspiratory muscles are in a permanently shortened position which creates a poor lengthtension relationship. Despite some adaptation of the muscles to
Table 1 SIGN (Scottish Intercollegiate Guidelines Network) Annex B: key to evidence statements and grades of recommendations

\begin{tabular}{ll}
\hline Levels of evidence \\
\hline $1++$ & $\begin{array}{l}\text { High-quality meta-analyses, systematic reviews of randomised controlled } \\
\text { trials (RCTs), or RCTs with a very low risk of bias }\end{array}$ \\
$1+$ & $\begin{array}{l}\text { Well-conducted meta-analyses, systematic reviews, or RCTs with a low risk } \\
\text { of bias }\end{array}$ \\
$1-$ & $\begin{array}{l}\text { Meta-analyses, systematic reviews, or RCTs with a high risk of bias } \\
2++\end{array}$ \\
High-quality systematic reviews of case-control or cohort studies \\
High-quality case-control or cohort studies with a very low risk of \\
confounding or bias and a high probability that the relationship is causal \\
Well-conducted case-control or cohort studies with a low risk of \\
confounding or bias and a moderate probability that the relationship is causal \\
Case control or cohort studies with a high risk of confounding or bias and a \\
significant risk that the relationship is not causal
\end{tabular}

Grades of recommendations

A At least one meta-analysis, systematic review, or RCT rated as $1++$, and directly applicable to the target population; or

A body of evidence consisting principally of studies rated as $1+$, directly applicable to the target population, and demonstrating overall consistency of results

B A body of evidence including studies rated as $2++$, directly applicable to the target population, and demonstrating overall consistency of results; or

Extrapolated evidence from studies rated as $1++$ or $1+$

C A body of evidence including studies rated as 2+, directly applicable to the target population and demonstrating overall consistency of results; or

Extrapolated evidence from studies rated as $2++$

D Evidence level 3 or 4 ; or

Extrapolated evidence from studies rated as $2+$

\section{Good practice points}

Recommended best practice based on the clinical experience of the guideline development group

Note: the grade of recommendation relates to the strength of the evidence on which the recommendation is based. It does not reflect the clinical importance of the recommendation.

this shortening, inspiration may need to be augmented by the accessory muscles of respiration, requiring fixation of the shoulder girdle. Although by fixing the shoulder girdle thoracic volume can be increased and ventilation improved, respiratory muscle oxygen consumption is increased. ${ }^{23}$ Patients therefore need to be taught how to fix the shoulder girdle and reduce ERV without increasing oxygen consumption.

\section{Level of evidence 4}

Recommendation

- Advise on passively fixing the shoulder girdle for optimising ventilatory muscle efficiency and relief of breathlessness. (Grade D)

Good practice points

- Elbows resting on knees or a table when seated, or on a suitable surface, for example a windowsill or wall, when standing.

- Hands/thumbs resting in/on pockets, belt loops, waistband, or across the shoulder handbag strap when ambulating.

One study compared lung function measurements in slumped and upright sitting. ${ }^{24}$ The position did not affect forced vital capacity (FVC), minute ventilation (MV), respiratory rate (RR), oxygen saturation $\left(\mathrm{SaO}_{2}\right)$ or forced expiratory volume in 1 second $\left(\mathrm{FEV}_{1}\right)$. In contrast, studies of forward lean sitting, which is with the patient's elbows or forearms resting on their knees or a table, have identified improvements in lung function ${ }^{25}$ with corresponding relief of dyspnoea, ${ }^{26-29}$ reduction in the work of breathing, ${ }^{28} \mathrm{ERV}$ and $\mathrm{MV}$, without any 
worsening of arterial blood gases (ABGs) ${ }^{25}$ and with reversal of paradoxical abdominal wall motion. ${ }^{26}$ These authors hypothesised that the forward lean sitting position facilitates diaphragmatic function by optimising its length-tension relationship, since upward force of the abdominal contents produces greater cephalad displacement of the diaphragm, lengthening the normally shortened muscle.

Whilst much of this work is old and methodologically weak, it does provide support for the observation that forward lean sitting relieves dyspnoea in many patients, in both the acute and chronic settings, and that the mechanics of breathing need to be carefully considered when choosing or advising positions for these patients.

\section{Level of evidence $2+$}

\section{Recommendation}

- Assess the effectiveness of forward lean sitting on relief of breathlessness in all patients with COPD, in both the chronic and acute settings. (Grade C)

Good practice point

- Combine shoulder girdle fixation and forward lean positioning.

In lying, diaphragm loading, utilising the abdominal contents as a fulcrum, also appears to have a positive influence on its force-generating capacity in this group of patients. Although, as in healthy subjects, maximal inspiratory pressure (MIP) and maximal expiratory pressure (MEP) are higher when sitting than when supine, in contrast to healthy subjects, transdiaphragmatic pressure is greater when supine. ${ }^{30}$

In side lying, the position of the lower limbs influences diaphragm loading. The conventional "recovery" position, with the uppermost hip and knee flexed, allows maximum unencumbered diaphragmatic excursion and "unloads" the diaphragm. This position may not be helpful in patients with COPD. In contrast, when the dependent hip and knee are flexed, this will tend to load the diaphragm and may lead to improved respiratory muscle function in the patient with hyperinflation. In standing, the same principles apply, using hip flexion to load the diaphragm. To date, studies have not been performed on patients with COPD to confirm the clinical effects of different lying or standing positions on the mechanics of breathing.

\section{Level of evidence 4}

Recommendation

- Advise modification of the forward lean position for use in standing and lying, for patients for whom forward lean sitting is effective. (Grade D)

Research recommendation

- Further research into the effects of position on ventilation, respiratory mechanics and clinical outcomes is required.

\section{Walking aids}

The combination of forward leaning and fixation of the shoulder girdle is achieved during ambulation with the use of a rollator frame (see Glossary, Appendix A), which has been shown to increase ventilatory capacity and walking distance, and to show a trend towards reduced fatigue and dyspnoea, in patients with severe COPD. ${ }^{31}$ Patients report that the use of a wheeled supermarket trolley greatly facilitates ambulation. In a four-way randomised controlled trial during hospital admission following acute exacerbation in an elderly COPD population, mobilisation using a rollator frame reduced physical disability, with the greatest improvement seen in those using a rollator gutter frame. ${ }^{32}$

\section{Level of evidence $1+$}

Recommendations

- Assess the effectiveness of a rollator frame for patients with COPD disabled by breathlessness. (Grade B)

- Assess the effectiveness of a gutter rollator frame in the acute setting, for patients with COPD severely disabled by breathlessness, especially the elderly. (Grade B)

\section{Energy conservation techniques}

Energy conservation techniques are commonly taught within the constructs of pulmonary rehabilitation (PR), but may be taught on an individual basis. Ideally this is supported by Occupational Therapy. Generally, energy conservation techniques consist of facilitating a reduction in the energy expenditure of a task, such as during activities of daily living. It involves organisation and pacing of tasks, as well as alternative ways to undertake them. This may be achieved via a change in posture, by teaching fixation of the shoulder girdle whilst undertaking tasks such as shaving or hair brushing, or sitting down to do simple chores, such as washing up.

Energy conservation also includes the use of appropriate home adaptations, such as a seat or hand rail, the lowering or raising of objects, or the use of small aids to reduce or eliminate high effort movement, for example a "helping hand" to reach for high objects, or a "sock aid". One recent small study showed a statistically significant benefit on dyspnoea of energy conservation techniques used during standardised activities of daily living. ${ }^{33}$ Pragmatically, it is sensible to combine energy conservation techniques with breathing techniques (see below).

\section{Level of evidence 4}

\section{Recommendation}

- Teach individualised energy conservation techniques to help reduce dyspnoea during activities of daily living. (Grade D)

\section{Breathing exercises}

It was recognised by physiotherapists many years ago that there were benefits to be gained from retraining patients' breathing pattern and that this would assist in exercise training. ${ }^{11}$ Several of the included studies identified dyspnoea as a secondary outcome or used breathing exercises as the control condition against another intervention, for example constant load cycle ergometry. Studies were mainly small with poorly defined patient characteristics. Most studies included focus on diaphragmatic breathing, pursed lips breathing and/or a combination of these two techniques. A review of breathing exercises in $\mathrm{COPD}^{34}$ concluded that the evidence for pursed lips breathing was sufficient to include its use, but the evidence for diaphragmatic breathing was not.

There are various other techniques which are grounded in physiological theory but which have little or no evidence base to support or refute them. However, these techniques are strongly endorsed by our patient representatives who report that breathing retraining is one of the most valuable aspects of rehabilitation. The techniques are commonly taught by physiotherapists to help patients cope with breathlessness and are often an integral part of the rehabilitation process, which includes management of patients admitted with acute exacerbations. Patients are advised to practise them when well and resting, so that they can be used when active and getting breathless. They are also advocated for acute dyspnoeic episodes. For further review of the available literature the reader is referred to Gosselink. ${ }^{35}$ 


\section{Breathing control}

Breathing control means breathing gently, using the least effort, with arms supported and shoulders and hands relaxed ${ }^{36}$ ideally using the forward lean sitting position for those with COPD. The patient is instructed to breathe gently and to try to feel more relaxed and calmer with each exhalation. ${ }^{37}$ It is commonly taught by physiotherapists in the UK to help patients to gain control of their breathing. It is equally useful in both acute and chronic care settings. It needs to be emphasised that it should not be confused with "diaphragmatic breathing".

\section{Level of evidence 4}

Recommendation

- Teach patients with COPD breathing control at rest to see if it helps relieve dyspnoea. (Grade D)

\section{Diaphragmatic breathing}

Diaphragmatic breathing is when outward motion of the abdominal wall, with minimal chest wall motion, is encouraged during inspiration, commonly by the placement of the therapist's or the patient's hand on the abdomen. This may be problematic for those with hyperinflation. Oxygen consumption $\left(\mathrm{VO}_{2}\right)$ and respiratory rate $(\mathrm{RR})$ were compared in 30 stable COPD subjects at rest, during usual breathing, and during pursed lips breathing, diaphragmatic breathing and a combination of the two. ${ }^{38} \mathrm{VO}_{2}$ and $\mathrm{RR}$ were lower during all three breathing techniques compared with usual breathing $(p<0.05)$, suggesting that these techniques may be beneficial.

Both Vitacca et $a l^{39}$ and Gosselink et al, ${ }^{40}$ however, showed that diaphragmatic breathing in severe COPD patients was associated with an increased sensation of dyspnoea. Although there were improvements in ABGs with diaphragmatic breathing, ${ }^{39}$ it was at the cost of greater inspiratory loading ${ }^{39}$ and poorer mechanical efficiency ${ }^{40}$ than usual breathing. A review of breathing exercises in $\mathrm{COPD}^{34}$ concluded that the evidence for diaphragmatic breathing was not sufficient to include its use in the management of patients with severe COPD.

\section{Level of evidence $2+$}

Recommendation

- Diaphragmatic breathing should not be taught routinely to patients with severe COPD. (Grade C)

\section{Pursed lips breathing}

$\mathrm{VO}_{2}$ and $\mathrm{RR}$ were compared in 30 stable COPD subjects at rest, during usual breathing, pursed lips breathing, diaphragmatic breathing and a combination of the two. ${ }^{38}$ Pursed lips breathing resulted in lower $\mathrm{VO}_{2}$ and $\mathrm{RR}$ than usual breathing $(\mathrm{p}<0.05)$. Garrod et $a l^{41}$ demonstrated that pursed lips breathing performed during exertion led to a reduced $R R$ and increased recovery rate compared with usual breathing. However, no differences in dyspnoea or exercise tolerance were found. A review of breathing exercises in $\mathrm{COPD}^{34}$ concluded that the evidence for pursed lips breathing was sufficient to include its use in the management of patients with COPD.

\section{Level of evidence $2+$}

\section{Recommendation}

- Teach pursed lips breathing during exertion as a potential strategy to reduce respiratory rate and aid recovery in patients with COPD. (Grade C)

\section{Exhalation on effort ("blow as you go!")}

The load of ventilation falls on the diaphragm when the shoulder girdle is moving, which many patients with COPD and hyperinflation are unable to cope with. ${ }^{23}$ Upper limb activities thus frequently exacerbate dyspnoea markedly. Moreover, the effort of inhalation creates a respiratory load in its own right. Patients therefore may have a tendency to breath-hold during exertion, which is counterproductive. This technique is aimed at avoiding both breath-holding and inhaling with effort. The patient is instructed to exhale when raising their arms above the head, bending down, stretching or on the greatest effort part of a movement, for example when standing up.

\section{Level of evidence 4}

Recommendation

- Teach exhalation on effort ("blow as you go!") as a potential strategy to reduce dyspnoea in patients with COPD. (Grade D)

Good practice point

- Breath-holding during exertion should be strongly discouraged.

\section{Relaxed, slower, deeper breathing}

Over 30 years ago, Motley ${ }^{42}$ demonstrated that slow, controlled breathing to a predetermined speed produced an increase in tidal volume $\left(\mathrm{V}_{\mathrm{T}}\right)$ and a reduction in the arterial partial pressure of carbon dioxide $\left(\mathrm{PaCO}_{2}\right)$. One physiological study suggested that this pattern of breathing may predispose to diaphragm fatigue when the expiratory time was also shortened in the imposed pattern. ${ }^{43}$ This technique is advocated, therefore, in combination with techniques to aid length of expiration (pursed lips breathing and "blow-as-yougo!"), for use during exertion. It is aimed at avoiding the tendency to adopt rapid shallow breathing, with subsequent inadequate alveolar ventilation.

It is important to emphasise that the change in rate and depth are in relation only to the patient's own natural pattern. The use of a pulse oximeter during exertion and tests of exercise tolerance are useful indicators of the efficacy of the technique. This technique is not advocated for an acutely breathless patient.

\section{Level of evidence 3}

Recommendation

- Teach relaxed, slower, deeper breathing as a potential strategy to facilitate more effective ventilation during exertion in patients with COPD. (Grade D)

Good practice point

- The use of this technique should be confined to during activity.

\section{Paced breathing}

The patient is instructed to inhale and exhale in time with steps, in a rhythm that suits them. It is thought to reduce dyspnoea during activity, for example when climbing the stairs or walking, and to help avoid rushing, breath-holding or rapid shallow breathing. It utilises a combination of the above techniques to suit the activity and the patient.

\section{Level of evidence 4}

\section{Recommendation}

- Teach paced breathing as a strategy to maintain control of breathing and reduce dyspnoea during exertion in patients with COPD. (Grade D)

Good practice point

- Consider combining techniques.

Research recommendation

- Further research into the use and effectiveness of different breathing strategies is required. 


\section{Ventilation feedback training}

Collins et al ${ }^{44}$ performed a three-way randomised controlled trial (RCT) comparing the effects of 6 weeks exercise training plus ventilation feedback training with those of exercise training alone or ventilation feedback alone. Ventilation feedback training offered no advantage over exercise training alone or benefit on exercise capacity, $\mathrm{MV}, \mathrm{V}_{\mathrm{T}}$ or breathing frequency in the absence of exercise. This work has been confirmed by a further recent study. ${ }^{45}$

\section{Level of evidence $2+$}

Recommendation

- Ventilation feedback training is not indicated in patients with COPD. (Grade C)

\section{Management of anxiety and panic attacks}

Good positioning, the breathing techniques above, relaxation and other strategies may be employed by physiotherapists to ameliorate anxiety and panic associated with breathlessness. Although there is no published evidence in COPD, this strategy is strongly endorsed by patient representatives. What little literature exists has been included under the management of asthma (see Section 2) and in the section on complementary therapies (Web Appendix 1).

\section{Level of evidence 4}

Recommendation

- Teach patients with COPD positioning, breathing and relaxation strategies to help manage anxiety and panic attacks. (Grade D)

\section{Pulmonary rehabilitation}

There are a significant number of RCTs investigating and demonstrating evidence of benefits of PR for patients with COPD. The European Respiratory Society (ERS) and American Thoracic Respiratory Society (ATS) recently adopted the following definition of PR: "an evidence-based multidisciplinary and comprehensive intervention for patients with chronic respiratory disease who are symptomatic and often have decreased daily life activities. Integrated into the individualized treatment of the patient, pulmonary rehabilitation is designed to reduce symptoms, optimize functional status, increase participation, and reduce health care costs through stabilising or reversing systemic manifestations of the disease" ${ }^{14}$

It is beyond the scope of this document to review the evidence concerning PR; however, several other recent systematic reviews and guidelines support its use. ${ }^{76} 47$ In essence, PR has proven benefits on exercise tolerance, dyspnoea and healthrelated quality of life, as well as improvements in healthcare utilisation and psychosocial outcomes. In addition, there is some evidence to support the fact that longer term rehabilitation, education and strength training are beneficial. ${ }^{46}$ The most recent guidelines ${ }^{46}$ make 25 recommendations concerning PR. All recommendations are of direct relevance to physiotherapy practice, with the majority at Grade A.

\section{Level of evidence $1++$}

Recommendations

- Pulmonary rehabilitation should include exercise training of the muscles of ambulation. (Grade A)

- Pulmonary rehabilitation should incorporate strength training of both upper and lower limbs. (Grade A)

- Information, advice and education should be integral to pulmonary rehabilitation. (Grade A)

- Pulmonary rehabilitation should be made available to all appropriate patients with COPD. (Grade A)
Good practice points

- Physiotherapists, trained as they are in exercise, breathing and pacing techniques for patients with a wide range of respiratory disease, should be central to the delivery of effective pulmonary rehabilitation.

- Physiotherapists involved in the delivery of pulmonary rehabilitation need to be familiar with current published guidance.

\section{Assessments of exercise tolerance}

The assessment of exercise tolerance, in particular field exercise tests, is of great relevance to physiotherapy, notably for PR, but also for use in other settings, such as on the ward or in the home. Although a review of the literature on these tests is outside the scope of these guidelines, it is worth reinforcing that studies of tests of exercise tolerance recommend the use of a practice walk. ${ }^{48-50}$

Recent studies have confirmed the importance of practice walks to eliminate potential clinical errors, such as the incorrect prescription of the level of endurance exercise, or incorrectly attributing improvements in distance walked to ambulatory oxygen. ${ }^{51}{ }^{52}$ Another potential for error by omitting a practice walk is falsely attributing or exaggerating improvements in test performance to the PR intervention, rather than to the learning effect of the test.

\section{Level of evidence $2+$}

\section{Recommendation}

- The recommended number of practice walks must be included when assessing exercise tolerance with a field exercise test for the prescription of either exercise or ambulatory oxygen. (Grade C)

\section{Pulmonary rehabilitation peri- and postexacerbation}

Evidence is emerging on the importance of instituting exercise as soon after exacerbation as possible. Improvements in 3month accident and emergency visits and hospital bed days, as well as exercise tolerance, were found in those who attended community-based PR early (within 10 days) postexacerbation, ${ }^{53}$ compared with a control group. Where PR was provided during a hospital at home scheme, those who received it had improved exercise tolerance, muscle strength and health-related quality of life, as well as a reduction in the number of subsequent exacerbations, compared with the control group..$^{54}$

A 2005 meta-analysis ${ }^{55}$ concludes that exercise training early in the course of exacerbation recovery, both inpatient and outpatient based, increases exercise capacity and health-related quality of life, with a trend towards a reduction in dyspnoea. Furthermore, there is some evidence that it may reduce the risk of hospital admission and mortality, but larger controlled trials of early intervention with exercise are required to confirm this. ${ }^{55} \mathrm{~A}$ more recent study ${ }^{56}$ of inpatient rehabilitation 5 days per week during admission for an acute exacerbation found improvements in both exercise tolerance and health-related quality of life.

\section{Level of evidence $1+$}

Recommendations

- Consider pulmonary rehabilitation soon after exacerbation for patients with COPD. (Grade B)

- Consider some form of rehabilitation during exacerbation to maintain mobility and function in patients with COPD. (Grade D)

\section{Inspiratory muscle training}

Patients with moderate to severe COPD have been shown to gain improvements from inspiratory muscle training (IMT) in a 
variety of outcomes: dyspnoea, exercise tolerance and healthrelated quality of life, as well as in inspiratory muscle strength and endurance. The studies focused primarily either on IMT alone or on IMT in combination with a full body exercise programme. Due to the nature of the studies, it is hard to unravel the additional benefits of IMT over and above, or relative to, conventional whole body exercise.

Inspiratory muscle training in conjunction with general body training Several papers have investigated the combination of IMT with exercise programmes of cycle ergometry training, ${ }^{57-66}$ although findings are inconclusive as studies are small, often methodologically weak and results are inconsistent. Berry et a ${ }^{65}$ conclude that there is no significant difference in exercise tolerance gains between IMT and IMT plus exercise. In contrast, Dekhuijzen et $a l^{58}$ found an improvement using the combination of IMT and PR. More recently, Larson et al ${ }^{66}$ identified that there was a reduction in dyspnoea from IMT combined with cycle ergometry training, but this did not transfer to dyspnoea experienced during daily activity. There is no evidence that IMT leads to reductions in the perception of fatigue in patients with COPD. ${ }^{66}$

A 2002 meta-analysis, ${ }^{67}$ suggested that IMT as an adjunct to a general body exercise programme does not result in statistically significant benefits in functional exercise tolerance, but this may be a feature of power. However, there were additional benefits of IMT on inspiratory muscle strength and endurance over and above that of general exercise alone, particularly evident where respiratory muscle weakness was identified at baseline.

Although in a 2008 update of this meta-analysis ${ }^{68}$ two new studies have been included, these do not significantly alter the strength of the recommendations. The authors conclude that performing a combination of IMT plus exercise may lead to significant improvements in inspiratory muscle strength and one outcome of exercise tolerance for individuals with COPD.

There remains insufficient evidence to support or refute the routine addition of IMT to a PR programme to increase benefit for patients with COPD.

\section{Level of evidence 1++}

\section{Recommendation}

- Consider adding inspiratory muscle training to a general exercise programme where respiratory muscle weakness is thought to be contributing to the patient's problems. (Grade A)

Research recommendation

- Further research is required to establish the adjunctive and relative efficacy of inspiratory muscle training with pulmonary rehabilitation.

\section{Respiratory muscle training alone}

In patients with moderate to severe COPD, IMT improved inspiratory muscle strength and endurance $e^{59-61} 67$ 69-73 and exercise tolerance, as measured by either the incremental shuttle walk test (ISWT), or 6 and 12 min walking distances ( 6 and 12 MWDs)..$^{56} 62697073$ Small but statistically significant improvements have been shown in RCTs for the $12 \mathrm{MWD}^{62}{ }^{70} 6 \mathrm{MWD}^{73}$ and the ISWT. ${ }^{60}$

A number of RCTs demonstrate improvements with IMT in dyspnoea, ${ }^{60} 6364697173$ and health-related quality of life, using valid and reliable measures. ${ }^{5960669}$ This may contribute to improvement in performance in activities of daily living. ${ }^{69}$ A 2005 metaanalysis $^{74}$ concluded that IMT results in improved inspiratory muscle strength and endurance compared with education, but that further trials were required to investigate the effect of IMT compared with other rehabilitation interventions for outcomes such as dyspnoea, exercise tolerance and quality of life.

\section{Level of evidence 1+}

Recommendation

- Consider inspiratory muscle training in the management of COPD to improve respiratory muscle strength and/or endurance. (Grade A)

Good practice points

- Inspiratory muscle training should not be used to replace pulmonary rehabilitation.

- Inspiratory muscle training should be considered for patients who are unwilling or unable to partake in pulmonary rehabilitation in order to improve dyspnoea and exercise tolerance.

\section{Maintenance of training}

Only one paper has addressed the maintenance of IMT, $^{73}$ concluding that unless a maintenance programme was followed the outcomes of IMT would deteriorate within a year.

\section{Level of evidence $2+$}

Recommendation

- Consideration of maintenance of an inspiratory muscle training programme is required. (Grade D)

\section{Respiratory muscle training modalities}

The types of training reported in the literature were targeted resistive training, threshold training, the test of incremental respiratory endurance (TIRE) and interval training. In an early review of IMT, ${ }^{57}$ it was identified that poor control of breathing pattern enabled the patient to alter the training pressures used when using resistive training methods. Targeted resisted training $^{58}$ addressed this issue and achieved more positive results.

\section{Level of evidence $2+$}

Recommendation

- Devices that incorporate control of breathing pattern and flow rate should be considered over devices that do not have this function. (Grade D)

\section{Respiratory muscle training frequency and intensity}

A wide range of training pressures were identified, from $15 \%$ to $80 \%$ of MIP, most frequently between $30 \%$ and $60 \%$ MIP Moreover, the length of training programmes also varied greatly, from 6 weeks to 6 months, the most common being 6-9 weeks, and these variations may have influenced outcomes. In one study comparing different intensities of training in COPD, the $6 \mathrm{MWD}$ was statistically significantly increased in the higher ( $30 \%$ of maximum), compared with the lower, intensity group. ${ }^{69}$

\section{Level of evidence 2 -}

Research recommendation

- Studies are required to establish the optimum frequency and intensity of training modalities and most efficacious maintenance therapy.

\section{Non-invasive ventilation (NIV)}

Physiotherapists have long used NIV, initially in the form of intermittent positive pressure breathing (IPPB), ${ }^{11} 75$ and are recognised as important researchers and clinicians in the delivery of NIV, including assessment and referral of patients, and administration and monitoring of the effects of treat- 
ment. ${ }^{15-20}$ Undergraduate training in physiotherapy includes the effects and delivery of NIV, and many centres have services either run by physiotherapists or with physiotherapist involvement. NIV has been shown to be an effective treatment for acute hypercapnic respiratory failure in COPD, but it is beyond the scope of these guidelines to review the literature concerning NIV, and the reader is referred to other published guidelines ${ }^{76376}$ and systematic reviews in this area. ${ }^{77-79}$

It should be noted that "staff training and support" are crucial for the effective delivery of NIV. ${ }^{76}$

\section{Level of evidence $1++$}

\section{Recommendations}

- Non-invasive ventilation should be offered to patients with COPD and acute hypercapnic respiratory failure, if they meet recommended BTS criteria. (Grade A)

- Facilities for non-invasive ventilation should be available $24 \mathrm{~h}$ per day in all hospitals likely to admit such patients. (Grade A)

Good practice points

- Personnel involved with the delivery and care of patients using non-invasive ventilation should be adequately trained in the principles, assessment and effects of non-invasive ventilation.

- Physiotherapists involved in the delivery of non-invasive ventilation need to ensure that their practice remains in line with current guidance.

\section{Intermittent positive pressure breathing}

The literature on IPPB is very old and has, by and large, been forgotten and discounted, both due to the advent of NIV and because many clinical trials used it inappropriately, with negative results. ${ }^{80-84}$ Like any form of NIV, however, IPPB provides positive pressure throughout inspiration and, if set up to ensure patient synchrony, will decrease the work of breathing. ${ }^{85}$ IPPB improves distribution of ventilation ${ }^{86} 87$ and ABGs when $V_{T}$ is increased. ${ }^{86-89}$ IPPB reduced the hypercapnia and acidosis associated with oxygen therapy when settings achieved a large increase in $\mathrm{V}_{\mathrm{T}}{ }^{89}$

\section{Level of evidence $2+$}

\section{Recommendations}

- Tidal volume must be increased to achieve a therapeutic effect. (Grade C)

- Care must be taken to ensure settings achieve patient synchrony with the device to reduce work of breathing. (Grade C)

\section{Intermittent positive pressure breathing in stable COPD}

Long-term daytime use of short periods of IPPB in stable COPD has been shown to be of no therapeutic value, ${ }^{81-84}$ and this method of application of IPPB does not reflect either physiotherapy clinical practice or current knowledge of effective use of NIV.

\section{Level of evidence $1++$}

Recommendation

- Short periods of daytime intermittent positive pressure breathing should not be used to treat chronic respiratory failure in stable COPD. (Grade A)

\section{Intermittent positive pressure breathing in acute exacerbation of COPD}

Although increasing $\mathrm{V}_{\mathrm{T}}$ and/or MV only temporarily has no place in the management of stable patients with COPD, the same is not true when the patient is acutely unwell. One study ${ }^{80}$ reported mixed success, but patients were treated in the supine position and, for those with acute exacerbation, with a lower mean respiratory rate (16.5) than for those free of symptoms (19) and without a significant improvement in $\mathrm{V}_{\mathrm{T}}$. This does not reflect current accepted clinical use of an NIV device.

IPPB is used by physiotherapists as a means of providing temporary assistance to ventilation when the patient is too weak or tired to increase $V_{\mathrm{T}}$ independently or effectively. ${ }^{11}$ It allows the fatigued patient better to tolerate and carry out airway clearance, which might otherwise be too tiring.

\section{Level of evidence 4}

Recommendation

- Consider intermittent positive pressure breathing in acute exacerbations of COPD where patients present with retained secretions but are too weak or tired to generate an effective cough. (Grade D)

Good practice point

- Intermittent positive pressure breathing may be considered in acute exacerbations of COPD where patients do not have immediate access to non-invasive ventilation and intubation is not an option.

\section{Fraction of inspired oxygen with intermittent positive pressure breathing}

Concerns have been raised regarding the use of IPPB in acute exacerbation of COPD because of the fraction of inspired oxygen $\left(\mathrm{FiO}_{2}\right)$ used, since IPPB is driven by compressed gas, usually oxygen..$^{0}$ Early work reported successful use of IPPB to reduce the hypercapnia and acidosis associated with oxygen therapy. ${ }^{89}$ It should be noted that a large increase in $V_{\mathrm{T}}$ was achieved in this study. In an RCT of COPD patients in acute respiratory failure, ${ }^{91}$ comparing IPPB with $\mathrm{FiO}_{2}$ of 0.24 or 0.4 , there was no difference in the partial pressure of carbon dioxide $\left(\mathrm{PaCO}_{2}\right)$ between groups, although hypoxaemia was better corrected in the $\mathrm{FiO}_{2} 0.4$ group.

\section{Level of evidence $1+$}

Recommendation

- When using intermittent positive pressure breathing in acute respiratory failure, $\mathrm{FiO}_{2}$ of 0.4 may be used. (Grade B)

Good practice point

- Monitor the patient carefully and ensure they are returned to their normal $\mathrm{FiO}_{2}$ following treatment.

\section{Oxygen therapy}

Physiotherapists are commonly involved in the delivery of oxygen therapy, and current BTS guidance should be considered, whether in the acute setting, ${ }^{92}$ the domiciliary setting ${ }^{93}$ or when assessing for or using ambulatory oxygen. ${ }^{93}$ In 60 stable COPD patients ${ }^{94}$ who randomly performed, on two consecutive days, a standardised $6 \mathrm{MWD}$ using a fullweight oxygen canister either transported in a small wheeled cart pulled by the patient (aid modality) or carried on the patient's shoulder, the distance walked, peak effort dyspnoea and leg fatigue scores were significantly different between walks $(p<0.05)$ in favour of the aid modality. Greater differences were recorded in the subgroup of patients walking $<300 \mathrm{~m}$ at baseline.

\section{Level of evidence $1+$}

Recommendations

- Administer oxygen therapy, in both the acute and domiciliary settings, according to current national guidance. (Grade A) 
- Consider assessing the benefit of a walking aid to transport the ambulatory oxygen, especially for the more disabled patient. (Grade B)

\section{Airway clearance techniques}

There are a wide variety of airway clearance techniques, including: the active cycle of breathing techniques, the forced expiration technique (FET), autogenic drainage, and positive expiratory pressure (PEP) and oscillating PEP devices (see Glossary, Appendix A). The body of evidence for airway clearance techniques is greatest in patients with CF (see Section 3)

In COPD, the active cycle of breathing techniques and autogenic drainage have been shown to be equally effective, with similar improvements in lung function. ${ }^{95} 96$ These techniques produced statistically and clinically significant improvements in pulmonary function, ABGs, exercise tolerance and dyspnoea. ${ }^{97}$ Autogenic drainage showed greater improvements in peak expiratory flow rate (PEFR) and $\mathrm{PaCO}_{2}$, while active cycle of breathing techniques resulted in greater improvements in $\mathrm{SaO}_{2}$. While these differences showed statistical significance, it is questionable whether these specific differences between techniques are of clinical significance. ${ }^{97}$

The FET, which includes huffing (see Glossary, Appendix A), is a part of the active cycle of breathing techniques and increasingly is also used as part of other airway clearance methods. It has been shown to be effective in clearing sputum in patients with $\mathrm{COPD}^{98}$ and, when combined with postural drainage, is more effective than coughing alone. ${ }^{99}$ The dynamic airway compression that the FET causes does not limit sputum clearance in COPD. ${ }^{100}$

PEP and oscillating PEP devices have been shown to be equally effective as traditional chest physiotherapy in sputum clearance, $^{79598101}$ and are recognised as useful techniques in the NICE guidelines on COPD. ${ }^{7}$ There may be a patient preference for PEP devices, with or without an oscillatory function, over traditional methods of postural drainage and manual techniques, due to the convenience they offer to the patient. ${ }^{95}$ No difference in benefit has been shown between devices in aiding sputum clearance. ${ }^{79598101}$ It should be noted however, that PEP has not been compared with other more modern techniques in COPD and its efficacy relative to, for example, active cycle of breathing techniques or autogenic drainage therefore is hard to establish.

\section{Level of evidence 1+}

\section{Recommendations}

- Consider the active cycle of breathing techniques (which includes the forced expiration technique), autogenic drainage and plain or oscillating positive expiratory pressure for patients with stable COPD who need an airway clearance technique to assist in the removal of secretions. (Grade $\mathrm{C}$ )

- Incorporate postural drainage only if it further aids clearance and has no detrimental effects. (Grade D)

Good practice point

- Consider patient preference in the selection of airway clearance techniques and devices in patients with COPD.

\section{Pelvic floor muscle training}

The degree of urinary incontinence has been shown to be greater in those with chronic cough due to COPD and CF, compared with a normal population with stress incontinence. ${ }^{102}$ Evidence of increased prevalence of stress incontinence, compared with a normal population, as yet exists only for patients with CF. ${ }^{103-106}$ In the COPD population there may be other factors, such as weak musculature, in addition to risk factors common in the general population, for example following pregnancy. Symptoms are probably under-reported and subjects are unlikely to seek help with the problem. The presence of urinary incontinence may impact on the individual's ability and/or willingness to perform certain activities, such as some exercises, or airway clearance and lung function manoeuvres, especially during exacerbations of pulmonary infection.

An unpublished study reported improved electromyograph (EMG) activity over both 1 and $20 \mathrm{~s}$, and a decrease in symptoms following a comprehensive programme of pelvic floor muscle exercises and electrical stimulation ${ }^{107}$ in a mixed population of COPD and CF patients. The improvements were maintained at 3 months. Voluntary contraction of the pelvic floor muscles just before and throughout a cough or huff, known as "The Knack", has been used successfully to reduce stress-related leakage of urine. ${ }^{108}$

While there is no robust evidence to advocate physiotherapeutic interventions in the treatment of urinary incontinence in COPD, these pilot data lend support to its efficacy, and assessment and treatment of this condition may help prevent or decrease the severity of urinary incontinence.

\section{Level of evidence 3}

Recommendations

- Patients with COPD should be questioned about their continence status. (Grade D)

- All patients with chronic cough, irrespective of continence status, should be taught to contract the pelvic floor muscles before forced expirations and coughing ("The Knack"). (Grade D)

- If problems of leakage are identified, patients should be referred to a physiotherapist specialising in continence. (Grade D)

\section{Complementary therapy}

For discussion of complementary therapy techniques, please see Web Appendix 1.

\section{SECTION 2 ASTHMA AND DISORDERED BREATHING (HYPERVENTILATION SYNDROME)}

\section{SECTION 2a Asthma}

\section{Introduction}

UK asthma guidelines first appeared in $1990^{109} 110$ and have been updated at intervals. ${ }^{111-114}$ A key feature of asthma is dyspnoea, the symptom that will bring many patients to the physiotherapist. A confounding factor is that many patients with asthma also suffer from hyperventilation syndrome (see Section 2b). The physiotherapist has a choice of treatment modalities available, many of which have been in use for a considerable period of time. ${ }^{115}$ Dyspnoeic patients are treated on an individual basis, with the therapist continuously altering treatment components in response to patient feedback. ${ }^{116}$ Standardising treatment protocols, therefore, in order to provide high-grade evidence for RCTs is fraught with difficulties.

This section reviews interventions used by physiotherapists on a regular basis, namely breathing re-training and exercises, physical exercise, respiratory muscle training and airway clearance techniques. These varied interventions provide wideranging levels of evidence. ${ }^{117}$ Other techniques used by physiotherapists but considered complementary or alternative, such as relaxation, acupuncture, pilates and yoga, are discussed in Web Appendix 1. 


\section{Breathing exercises in asthma}

A 2000 systematic review of breathing techniques ${ }^{118}$ concluded that too few studies had been carried out to warrant firm judgments, but that collectively the data implied that physiotherapeutic breathing techniques may have some potential benefit. A 2004 Cochrane review of breathing exercises for asthma $^{117}$ concluded that, due to the diversity of breathing exercises and outcomes used, it was impossible to draw conclusions from the available evidence. The Cochrane review stated that trends for improvements, noted in a number of outcomes, warranted large-scale studies in order to observe their effectiveness in the management of asthma. Slader et al in $2006^{119}$ carried out a double-blind RCT of breathing techniques in asthma and concluded that breathing techniques may be useful in patients with mild asthma who use a reliever inhaler frequently. They found no evidence to favour nasal breathing over non-specific upper body exercises.

Three large RCTs have since been completed. ${ }^{120-122}$ A 2007 $\mathrm{RCT}^{120}$ demonstrated that breathing retraining and relaxation significantly reduced respiratory symptoms and improved health-related quality of life in a cohort of patients with asthma. A 2008 RCT $^{121}$ adds further strong support to this work, also finding significant reductions in asthma symptoms. The most recent RCT ${ }^{122}$ controlled for placebo effect by offering the control group exactly the same time with a healthcare professional-that is, an experienced respiratory nurse providing asthma education. There were significant improvements in asthma-related quality of life in both groups after 1 month, but at 6 months a large difference between groups was found, in favour of breathing exercises, in asthma quality of life, anxiety and depression, Nijmegen score and a trend for an improvement in asthma control. No effect on airway inflammation was found. Monitoring of the effect of treatment is important, as is the understanding that this form of therapy does not replace usual medical care.

\section{Level of evidence $1++$}

\section{Recommendations}

- Breathing exercises, incorporating reducing respiratory rate and/or tidal volume, and relaxation training, should be offered to patients to help control the symptoms of asthma and improve quality of life. (Grade A)

- The use of suitable tools such as an asthma-specific quality of life measure, measures of anxiety and depression, and the Nijmegen Questionnaire should be used to establish baseline severity of symptoms and monitor progress with treatment (Grade B).

Good practice point

- Patients should be advised that breathing strategies are adjunctive to, not replacement therapy for, medication.

\section{Buteyko breathing technique in asthma}

Cooper et al ${ }^{123}$ compared the Buteyko breathing technique (see Glossary) and yogic breathing exercises, concluding that the Buteyko breathing technique could improve symptoms and bronchodilator use but did not have an effect on lung function. A single-blind RCT ${ }^{124}$ comparing the Buteyko breathing technique with "conventional breathing techniques" (deep breathing) in asthma concluded that practising the Buteyko breathing technique reduced hyperventilation and $\beta_{2}$-agonist use, with a nonsignificant trend towards reduced steroid use and improved quality of life. The results of this study should be interpreted with caution; the control condition of deep breathing exercises is not designed to reduce ventilation but to increase it.
The 2008 RCT of Cowie et a ${ }^{121}$ had a more suitable control condition of appropriate breathing exercises aimed at reducing tidal volume and or respiratory rate, carried out by a physiotherapist. This study demonstrated that both the Buteyko breathing technique and the physiotherapy breathing exercises improved asthma control, which was maintained for 6 months. The only difference was a reduction in inhaled steroid use in the Buteyko group. However, in a recent review, ${ }^{125}$ a trend towards a reduction in medication use in the study of Bowler et a l $^{124}$ was noted as an inappropriate outcome, given that the Buteyko breathing technique includes active encouragement to reduce medication use. This review ${ }^{125}$ also reported a lack of evidence that changing breathing patterns can raise the partial pressure of arterial carbon dioxide $\left(\mathrm{PaCO}_{2}\right)$, the proposed mechanism behind the Buteyko breathing technique, as many studies included in their review did not measure $\mathrm{CO}_{2}$. No harmful effects were observed.

In a recent pilot study examining the effects of mouth taping, ${ }^{126}$ one of the components of the Buteyko breathing technique to encourage nose breathing, there was a trend for end-tidal $\mathrm{CO}_{2}$ to be increased during the mouth taping phase in nine mildly asthmatic subjects.

\section{Level of evidence 1+}

\section{Recommendation}

- The Buteyko breathing technique may be considered to help patients to control the symptoms of asthma. (Grade B)

Good practice point

- Consider any cost implications to the patient of the Buteyko breathing technique.

\section{Physical training in asthma}

There are a number of studies that have addressed the efficacy and effectiveness of physical training in asthma.

A recent review ${ }^{127}$ concluded that physical training improves cardiopulmonary fitness, but has no effect on resting lung function or number of days with wheeze. As 11 out of the 13 studies included in the review were conducted in children, the results cannot be extrapolated directly to adults. In one of the studies of adults, ${ }^{128}$ patients were randomised either to a physical training programme three times weekly for 3 months, or to a control arm of educational sessions. There was a significant improvement in the training group compared with the control group in mean maximal oxygen uptake $\left(\mathrm{VO}_{2}\right.$ max), and significant reductions in breathlessness, blood lactate and $\mathrm{CO}_{2}$ output. The mechanism for the reduced breathlessness appeared to be a reduction in maximal minute ventilation when exercising at high intensity. In the second study of adults, ${ }^{129}$ patients undergoing 3 months of thrice-weekly community-based rehabilitation, versus a 3 -month control period, had significant improvements in endurance cycling time, $6 \mathrm{MWD}$ and health-related quality of life.

There are several non-randomised studies ${ }^{130-133}$ supporting the use of physical training in asthma. One study ${ }^{130}$ found that 43 patients with asthma, following an intensive rehabilitation programme (training for $38 \mathrm{~h}$ a week for 3 months), still had significant improvements in $\mathrm{VO}_{2}$ max at 24 months compared with baseline values. Other less robust controlled studies have demonstrated improvements in exercise capacity. ${ }^{134} 135$ Several non-systematic reviews ${ }^{136-139}$ all support the use of exercise in the management of asthma, as do the patient representatives.

\section{Level of evidence $1+$}




\section{Recommendations}

- Physical training should be advised for improvements in fitness and cardiorespiratory performance in patients with asthma. (Grade B)

- Physical training should be advised to help reduce breathlessness and improve health-related quality of life in people with asthma. (Grade B)

- Physical training programmes should aim to reach a minimum of activity as per the American College of Sports Medicine guidelines. (Grade A)

\section{Inspiratory muscle training in asthma}

A Cochrane review ${ }^{377}$ concluded that there was insufficient evidence to suggest that IMT provides any clinical benefit in asthma.

An improvement in MIP was reported in three studies, but it is uncertain whether this translates to any clinical benefit. A reduction in the perception of dyspnoea was noted in two studies. ${ }^{140} 141$ There is insufficient evidence to support or refute the use of IMT in the management of asthma.

\section{Level of evidence 1 -}

Research recommendation

- Further research is required to ascertain clinical relevance of inspiratory muscle training in this patient group.

\section{Airway clearance techniques in asthma}

Few asthmatics warrant aggressive airway clearance techniques on a regular basis since sputum production is not always present.

When it is present and the patient experiences problems with clearance and expectoration, an appropriate technique may be required, but there is scant evidence on the best technique to use. Possible techniques include those described in Section 3 (Cystic fibrosis), since most evidence on airway clearance techniques has been collected in this client group. In any condition today, the emphasis is on teaching techniques that allow independence, rather than those that rely on carer help, and rarely include postural drainage, since this requires the sputum to be of sufficient quantity and viscosity to respond to gravity to increase its flow up the bronchial tree. Techniques that have been investigated specifically in asthma and which are therefore discussed in this section include: chest wall percussion (clapping), vibrations, shaking (collectively termed manual therapies), postural drainage, FET and directed coughing.

Barnabé et $a l^{142}$ investigated the safety of various chest physiotherapy techniques (including percussion, vibrations, shaking, postural drainage, FET and coughing) and concluded they are safe in stable asthma, including in those with severe symptoms. They concluded, however, that further study was needed in episodes of exacerbation to establish safety. One small study ${ }^{143}$ found non-significant improvements in pulmonary function with "physiotherapy" of undetermined technique, and a Cochrane review ${ }^{144}$ of manual therapies in asthma investigated a wide range of techniques (including percussion and massage) but found insufficient evidence to support or refute their use.

\section{Airway clearance adjuncts/devices}

Little evidence exists to support the use of PEP or oscillating PEP devices in asthma.

One crossover study ${ }^{145}$ found a significant improvement in ease of expectoration after 6 days of oscillating PEP use, but no objective changes in lung function or salbutamol use. Aitken et $a l^{146}$ also found no significant changes in pulmonary function, but cautioned clinicians using airway vibrations to be aware of the potential for bronchospasm as one subject had an asthma attack during the study. Tsai and Tsai ${ }^{147}$ reported significant improvements in lung function with PEP after nebulised $\beta_{2}$-agonist use in a small nonrandomised crossover study; results therefore should be considered with caution. Patient representatives report great benefit from steam inhalations, but no evidence to support their use could be found. There is insufficient evidence to support or refute the use of airway clearance techniques, including manual therapies and devices such as PEP in patients with asthma.

\section{Level of evidence 1 -}

Research recommendations

- Further research is required for the evaluation of airway clearance techniques in the management of secretions in asthma.

- Research into the effects of steam inhalations in asthma is required.

Good practice points

- Where an airway clearance technique is required, consider trying the simplest technique with the least effect on airway constriction.

- Monitor the patient's condition carefully throughout treatment.

Heart rate variability biofeedback in asthma

One RCT ${ }^{148}$ examined the use of heart rate variability biofeedback in asthmatics and found a significant decrease in medication use, a decrease in asthma severity level and an improvement in pulmonary function.

\section{Level of evidence 2-}

Research recommendation

- Further research is required in the evaluation of biofeedback in the form of heart rate variability in asthmatics.

\section{Complementary therapy}

Patients and patient representatives on these guidelines report benefit form certain complementary therapies, especially those that use controlled breathing techniques with exercise. For discussion of the use of these techniques in asthma, please see Web Appendix 1.

\section{SECTION 2b Disordered breathing (hyperventilation syndrome and vocal cord dysfunction)}

\section{Hyperventilation syndrome}

Hyperventilation syndrome for the purpose of this document may be defined as abnormal breathing that results in the sensation of breathlessness, often described as "air hunger", a sensation of an inability to take an apical breath, which may present with other symptoms; for example, chest tightness, anxiety, dizziness or paraesthesiae. These symptoms collectively characterise a syndrome, commonly referred to as hyperventilation syndrome or dysfunctional breathing. ${ }^{149}$ Many asthmatics hyperventilate chronically and can be treated, therefore, in the same way.

\section{Breathing techniques}

Physiotherapists have advocated breathing retraining for the management of disordered breathing for some time. ${ }^{12} 13$

Since symptoms arise from overbreathing, the focus of treatment in hyperventilation syndrome is to reduce the rate and depth of breathing, as opposed to conventional breathing exercises that aim to increase ventilation. In a 2007 RCT, ${ }^{120}$ 
breathing retraining and relaxation significantly reduced respiratory symptoms and improved health-related quality of life in a cohort of patients with asthma. The method of treatment used focused on the problems of dysfunctional breathing including hyperventilation.

An earlier RCT ${ }^{150}$ also reported that subjects taught diaphragmatic breathing exercises maintained a clinically relevant improvement in health-related quality of life for 9 months following the intervention. An old review with no published methodology ${ }^{151}$ reported that hyperventilation syndrome attacks were eliminated with therapy in a cohort of 106 outpatients in 1975. A pilot study ${ }^{152}$ on the effect of four treatments (stress reduction, breathing control, relaxation and hyperventilation provocation therapy) on different subjects concluded that all treatments were equally effective in reducing the number of symptoms, frequency of attacks and degree of depression.

A controlled study of breathing therapy for hyperventilation syndrome ${ }^{153}$ found that 10 weeks of breathing exercises, via a device to slow breathing, produced improvements in psychological factors, symptom complaints and respiratory parameters. A descriptive study ${ }^{154}$ found that $67 \%$ of 200 patients overbreathed and that "physiotherapy" was effective, but did not include details of any intervention. DeGuire et $a l^{155}$ also found that breathing retraining therapy improved respiratory parameters and was effective in reducing cardiac symptoms, with effects still present at 3 year-follow-up. ${ }^{156}$ Han et a ${ }^{157}$ concluded that in 92 subjects with a clinical diagnosis of hyperventilation syndrome, breathing retraining by a physiotherapist reduced anxiety and altered breathing frequency.

\section{Level of evidence 1+}

\section{Recommendation}

- Breathing retraining incorporating reducing respiratory rate and/or tidal volume should be offered as a first-line treatment for hyperventilation syndrome, with or without concurrent asthma. (Grade B)

Good practice point

- A suitable tool such as the Nijmegen Questionnaire should be used to establish baseline severity of symptoms and monitor progress with treatment.

\section{Vocal cord dysfunction}

Vocal cord dysfunction can be characterised as an abnormal adduction of the vocal cords during the respiratory cycle (especially during the inspiratory phase) that produces air flow obstruction at the level of the larynx.

Breathing retraining and relaxation, especially of the larynx, are described in the sports literature and advocated as possible useful therapies, concomitantly with psychological support in difficult cases. ${ }^{158}$ No primary research evidence was found to support or refute the use of these techniques, but members of the standards of care committee supported their use in this condition.

Research recommendation

- Research is required to establish the clinical efficacy of breathing techniques in vocal cord dysfunction.

\section{SECTION 3 CYSTIC FIBROSIS}

\section{Introduction}

CF is the most common life-threatening genetic disease in the Caucasian population, and is found in other ethnic groups. It is a multisystem disorder, although the main cause of morbidity and mortality is lung disease. ${ }^{159}$ The median life expectancy has increased significantly over the last two decades and is currently in the mid-thirties. ${ }^{160}$

Physiotherapy is an integral part of the management of the person with CF. The physiotherapist should provide holistic care including assessment and treatment for cardiovascular fitness (exercise), airway clearance, inhalation, oxygen therapy and NIV. Physiotherapists should also undertake assessment and treatment for musculoskeletal and postural disorders, and incontinence.

\section{Adherence}

A detailed review of the literature on adherence to treatment, although very pertinent to physiotherapy in this client group, is beyond the scope of these guidelines, but is nonetheless an important consideration in deciding upon the most appropriate technique for each patient. The concept of adherence evolved in order to reduce value judgements associated with the concept of compliance, and may be defined as "the extent to which a person's behaviour; taking medication, following a diet, and/or executing lifestyle changes, corresponds with agreed recommendations from a health care provider". ${ }^{161}$

A limited number of studies have reported on adherence in adults with CF. Most are of an exploratory nature and employ self-reports, physician reports, medical record reviews or prescription collections as their measurement strategy. ${ }^{162}$ These measurement strategies are problematic, raising concerns about the accuracy of the information which is likely to overestimate the extent of true adherence. ${ }^{162}$ Non-adherence to treatment is one of the major problems in the management of CF. ${ }^{163}$ Treatment factors such as the amount of time and effort, infringement on daily activities and unpleasantness are factors that may affect adherence. ${ }^{164}$

The patient preference for each technique is included within these guidelines where the data are present in the studies available.

\section{Level of evidence 4}

Research recommendations

- Further research, using validated methods, is required into adherence to physiotherapy interventions.

- Research into all physiotherapy techniques should include validated outcome measures to assess adherence levels and patient preference for technique.

\section{Exercise}

Exercise, or physical training, is the participation in a programme of regular vigorous physical activity designed to improve physical function, cardiovascular performance or muscle strength.

A Cochrane review ${ }^{159}$ demonstrated the positive effects of training programmes on exercise capacity, strength and lung function with aerobic or anaerobic physical training, in both short-and long-term trials, in people with CF. These improvements were not consistent among included studies and it has yet to be determined whether aerobic, anaerobic or a combination of both is optimal. Physical training is a routine part of the care package offered to most patients with CF, and there is no evidence to discourage this. ${ }^{159}$

The studies analysed within the review ${ }^{159}$ had a training protocol sufficient to obtain a training effect in deconditioned individuals. This was deemed to be a progression towards 20-30 min of exercise, at 55-64\% maximum heart rate, 3-5 times a week as recommended by the American College of Sports Medicine (ACSM) guidelines for exercise testing and 
prescription. ${ }^{165}$ The authors describe limitations in the present research base which prevent firm conclusions being drawn regarding exercise in this patient group. Studies to date are small scale, short duration and/or with incomplete reporting, and are not blinded due to the nature of the intervention.

\section{Level of evidence $1+$}

\section{Recommendations}

- Exercise should be an integral part of the management of patients with cystic fibrosis. (Grade B)

- Physical training programmes should aim to reach a minimum of activity as per the American College of Sports Medicine guidelines. (Grade A)

Research recommendations

- Further research is required to assess comprehensively the benefits of exercise programmes in patients with cystic fibrosis, particularly long-term effects.

- Further research is required on the relative benefits of aerobic and strength training for patients with cystic fibrosis.

\section{Exercise in patients with cystic fibrosis complications}

There was no identifiable evidence to guide prescription of training programmes in patients with CF complications such as osteoporosis or CF-related diabetes. There was a suggestion that physical training may aid the management of CF-related diabetes, delay onset of osteoporosis and lead to improved body image and decreased anxiety. ${ }^{159}$ The lack of data regarding adapting training programmes in view of comorbidities makes it vital that the specialist multidisciplinary team is involved in instigating and progressing exercise in an appropriate and safe manner.

\section{Level of evidence 4}

Good practice point

- Involve the specialist multidisciplinary team in the decision to instigate or progress physical training programmes in the adult with cystic fibrosis, especially in the presence of comorbidities.

Research recommendation

- Research is required to assess effects of exercise programmes in people with comorbidities such as osteoporosis and diabetes.

\section{Airway clearance techniques}

Mucociliary clearance can be augmented by an airway clearance technique.

A 2000 Cochrane review ${ }^{166}$ to determine the effectiveness of physiotherapy for airway clearance, compared with no physiotherapy and spontaneous cough, concluded that airway clearance techniques have short-term effects of increasing mucus transport.

A 2005 Cochrane review of airway clearance techniques in people with $\mathrm{CF}^{167}$ compared "conventional chest physiotherapy" (postural drainage, percussion, chest shaking, huffing and directed coughing) (see Glossary, Appendix A) with other techniques (PEP, high-pressure PEP (Hi-PEP), the active cycle of breathing techniques, autogenic drainage, oscillating PEP, highfrequency chest wall oscillation and exercise) (see Glossary). It concluded that there were no differences between "conventional chest physiotherapy" and more contemporary airway clearance techniques in affecting lung function, although all techniques improved lung function during an infective exacerbation. There was a trend for patients to favour selfadministered techniques, which may improve adherence to airway clearance.
There is insufficient evidence to support or refute the longterm use of airway clearance techniques in adults with CF, but there is an indication that physiotherapy has a major influence in limiting the adult consequences of CF. ${ }^{168}$

\section{Level of evidence $1+$}

\section{Recommendations}

- Teach patients with cystic fibrosis an airway clearance technique to increase mucus transport in the short term. (Grade A)

- Self-administered techniques should be the first-line airway clearance techniques offered in order to improve adherence to treatment. (Grade B)

- Patient preference for techniques should be considered in order to improve adherence to treatment. (Grade B)

Good practice points

- The technique that is simplest and most effective for any individual should be the method of choice.

- The frequency and duration of the airway clearance technique should be specific to the needs of the individual patient, which may alter with periods of infective exacerbation.

- When possible, the airway clearance treatment session should be undertaken until most of the excess bronchopulmonary secretions are expectorated.

- The airway clearance session should not be so long that the patient becomes fatigued.

Research recommendation

- Further research is required to assess the long-term effects of airway clearance techniques in adults with cystic fibrosis.

\section{Active cycle of breathing techniques}

The active cycle of breathing techniques combines deep breathing with the FET (see Glossary, Appendix A). Many early studies describing the FET were, in fact, referring to the active cycle of breathing techniques, which has caused some confusion.

The active cycle of breathing techniques can be used with or without manual techniques and postural drainage. When compared with deep breathing alone, with both regimens including postural drainage and manual techniques, it increases the rate of sputum expectoration, reducing the time required for airway clearance. ${ }^{169}$ In two small studies it has been found to be as effective when performed independently as it is with assistance, in terms of both energy expenditure ${ }^{170}$ and the amount of sputum expectorated, ${ }^{169}$ but with an assistant it also improved pulmonary function. ${ }^{170}$

The active cycle of breathing techniques alone clears more sputum than when used in combination with one oscillating PEP device. ${ }^{171}$ However, when huffing (see Glossary, Appendix A) is included in the oscillating PEP regimen, the two are equivalent. ${ }^{172}$ The active cycle of breathing techniques was less effective when used in combination with PEP, ${ }^{173}$ with or without gravity-assisted positioning, than when used alone.

The active cycle of breathing techniques with gravity-assisted positioning is as effective as autogenic drainage, either sitting or supine, in clearing sputum in the short term ${ }^{174}$ and as effective as autogenic drainage, PEP or two different oscillating PEP devices over a period of 1 year. ${ }^{175}$

\section{Level of evidence $1++$}

\section{Recommendation}

- Consider the active cycle of breathing techniques when recommending an airway clearance technique for adults with cystic fibrosis. (Grade A) 


\section{Autogenic drainage}

In one short-term RCT, ${ }^{174}$ autogenic drainage (see Glossary, Appendix A) was found to be as effective, but quicker than, the active cycle of breathing techniques in clearing sputum in patients with CF. In two long-term trials there were no significant differences in either sputum clearance or respiratory function between autogenic drainage and postural drainage with percussion, ${ }^{176} 177$ although patients preferred autogenic drainage. One study comparing Hi-PEP with autogenic drainage found improved clearance with Hi-PEP but improved lung function following autogenic drainage. ${ }^{178}$ Autogenic drainage has been shown to be as effective as one oscillating PEP device in altering sputum rheology. ${ }^{179}$ Autogenic drainage is as effective as the active cycle of breathing techniques, PEP or two different oscillating PEP devices over a period of 1 year. ${ }^{175}$

\section{Level of evidence $1++$}

Recommendation

- Consider autogenic drainage when recommending an airway clearance technique for adults with cystic fibrosis. (Grade A)

\section{Positive expiratory pressure}

One study ${ }^{180}$ found PEP (see Glossary, Appendix A) to be as effective as postural drainage with percussion. A Cochrane review $^{181}$ of PEP devices compared with other forms of physiotherapy, as a means of improving mucus clearance, concluded that there was no clear evidence that PEP was a more or less effective intervention. PEP is as effective as the active cycle of breathing techniques, autogenic drainage or two different oscillating PEP devices over a period of 1 year. ${ }^{175}$ The studies analysed used different forms of PEP devices, mask or mouthpiece, but there is no evidence to suggest any differences in effectiveness between the two.

\section{Level of evidence $1++$}

\section{Recommendation}

- Consider positive expiratory pressure when recommending an airway clearance technique for adults with cystic fibrosis. (Grade A)

There was limited evidence that PEP was preferred by participants, but this was from studies of low quality included in the Cochrane review. ${ }^{181}$

\section{Level of evidence 1 -}

Research recommendation

- Further research is required to assess patient preference for positive expiratory pressure as compared with other airway clearance techniques.

\section{Oscillating positive expiratory pressure}

A number of devices are available to provide oral oscillatory PEP. One device has been shown to be as effective as autogenic drainage in altering sputum rheology ${ }^{179}$ and as effective as postural drainage with percussion in short-term trials. ${ }^{182} 183$ Two devices have been shown to be as effective as the active cycle of breathing techniques, autogenic drainage or ordinary PEP over a period of 1 year, ${ }^{175}$ with nothing to suggest any difference in effectiveness between the two. There is insufficient evidence to assess the comparative effectiveness of the third currently available device.

\section{Level of evidence $1++$}

\section{Recommendation}

- Consider oscillating positive expiratory pressure devices when recommending an airway clearance technique for adults with cystic fibrosis. (Grade A)
High-pressure positive expiratory pressure

One study ${ }^{178}$ compared Hi-PEP (see Glossary, Appendix A) and autogenic drainage for a single treatment session only. More sputum was cleared with Hi-PEP than with either autogenic drainage or a combination of ordinary PEP and autogenic drainage. If used, treatment with Hi-PEP must be assessed regularly, by a physiotherapist skilled in the technique, owing to the high pressures used $\left(40-100 \mathrm{~cm} \mathrm{H}_{2} \mathrm{O}\right){ }^{184}$

There is insufficient evidence to support or refute the use of Hi-PEP in cystic fibrosis.

\section{Level of evidence 2-}

Good practice point

- Caution should be exercised and regular monitoring undertaken with high-pressure positive expiratory pressure.

Research recommendations

- Further research is required to establish the safety of highpressure positive expiratory pressure.

- Further research is required to establish the efficacy of highpressure positive expiratory pressure relative to other techniques.

\section{Postural drainage (gravity-assisted positioning)}

The effects of gravity on airway clearance are thought to be a consequence of both drainage and an increase in ventilation. ${ }^{180}$

Postural drainage with percussion has been shown to be as effective as intrapulmonary percussive ventilation, ${ }^{185}{ }^{186}$ with a suggestion that there may be patient preference for intrapulmonary percussive ventilation. ${ }^{186}$ The studies of intrapulmonary percussive ventilation included only subjects with mild to moderate disease and who were clinically stable.

Postural drainage with percussion has also been shown to be as effective as oscillating PEP in the short term ${ }^{182} 183$ and autogenic drainage in the long term, ${ }^{176} 177$ although patients preferred autogenic drainage.

Early studies of the active cycle of breathing techniques included postural drainage and manual techniques. ${ }^{169}$

Due to the higher viscosity of CF sputum today, however, and the move towards more user-friendly and independent techniques which encourage adherence, specific positioning for people with CF is likely to be of significance only, for example, in the drainage of an identified lung abscess or specific areas of atelectasis.

\section{Level of evidence $2+$}

Recommendation

- Individually assess the effect and acceptability of gravityassisted positioning in patients with cystic fibrosis. (Grade B)

\section{Modified postural drainage}

Treatment with the active cycle of breathing techniques in a modified postural drainage position (side lying), compared with conventional postural drainage (head-down tilt), produced no significant differences in lung function or in weight of sputum expectorated. The study population $(n=19)$ was a combination of CF- $(n=14)$ and non-CF-related bronchiectasis patients, most of whom preferred the horizontal position and felt less breathless without a head-down tilt. ${ }^{187}$

\section{Level of evidence $2+$}

\section{Recommendation}

- Individually assess the effect and acceptability of modified gravity-assisted positioning in individual patients with cystic fibrosis. (Grade C)

\section{Manual techniques}

Manual techniques include percussion (chest clapping) over the chest wall and chest shaking or vibrations (compression of the 
chest with coarse or fine oscillations, respectively) (see Glossary for all terms), with the aim of loosening bronchial secretions. They are most typically used in the UK in conjunction with the active cycle of breathing techniques. There are few papers which specifically investigate the effects of any of these techniques on mucus transport in CF. These techniques are typically used when the patient is unwell, or fatigued and needing help with their usual regimen.

McCarren and Alison ${ }^{188}$ compared the expiratory flow rates and frequencies of air flow oscillation using chest wall vibration, percussion, PEP and two oscillating PEP devices in subjects with CF. They concluded that although the oscillating PEP devices produced higher oscillation frequencies than chest wall vibration and percussion, chest wall vibration produced greater expiratory flow rates and a higher peak expiratory/peak inspiratory flow ratio.

The same authors conducted a within-subject, experimental study in three healthy subjects. ${ }^{189}$ Seven experienced cardiopulmonary physiotherapists applied various manual treatments in random order during passive expiration: chest vibration, compression alone and oscillation alone. Change in intrapleural pressure was compared with that during passive expiration alone. During vibration, $13 \%$ of the change in intrapleural pressure was attributed to compression, $12 \%$ to oscillation and $75 \%$ to lung recoil. They concluded that changes in intrapleural pressure occurring during vibration appear to be the sum of changes from lung recoil and the components of the technique, suggesting that all three factors are required to optimise expiratory flow.

\section{Level of evidence $2+$}

\section{Recommendation}

- If patients using independent techniques are unable to clear secretions effectively, chest wall vibration should be considered. (Grade C)

Thoracic cage compression as part of autogenic drainage during the expiratory phase can be used to facilitate lower lung volume level breathing and provides feedback for both the patient and physiotherapist. There is no evidence to support or refute the use of chest wall vibrations or shaking in this way.

A small-scale trial ${ }^{170}$ demonstrated that the addition of physiotherapist-performed percussion and vibration to postural drainage and the active cycle of breathing techniques had no significant effect on energy expenditure, but did reduce airways obstruction, in patients with CF and mild pulmonary impairment.

There is insufficient evidence to support or refute the routine use of chest wall percussion in patients with CF.

\section{Level of evidence 2 -}

Good practice point

- If patients using independent techniques with chest wall vibrations are unable to clear secretions effectively, percussion or chest shaking should be considered.

Research recommendation

- Further research is required into the effects of manual techniques in patients with cystic fibrosis.

\section{Mechanical devices for airway clearance Intrapulmonary percussive ventilation}

Intrapulmonary percussive ventilation provides a constant mean airway pressure maintaining partial inspiration, while internally percussing the airways, via high-flow jets of gas delivered by a pneumatic flow interrupter. This is combined with a side stream of room air and nebulised medication via a jet venturi. ${ }^{185}$ A Cochrane review concluded that the short-term use of intrapulmonary percussive ventilation is as effective as alternative techniques. ${ }^{167}$ There is a suggestion that there may be patient preference for intrapulmonary percussive ventilation over postural drainage and percussion. ${ }^{186}$ One study ${ }^{185}$ looked at the longer term effects over a period of 6 months, and concluded that intrapulmonary percussive ventilation was as effective as postural drainage and percussion.

\section{Level of evidence $1++$}

Recommendation

- Consider intrapulmonary percussive ventilation when recommending an airway clearance technique for adults with mild to moderate cystic fibrosis. (Grade A)

The above studies included only subjects with mild to moderate disease and who were clinically stable. There is little known of the effects of intrapulmonary percussive ventilation on patients with severe disease and/or with an exacerbation of pulmonary infection. Research recommendation

- Research is required to assess the effects of intrapulmonary percussive ventilation in patients with severe disease or when experiencing an infective exacerbation.

\section{High-frequency chest wall compression/oscillation}

High-frequency chest wall compression/oscillation can be administered via a pneumatic vest. This fits snugly over the thorax and is connected to an air-pulse generator. Intermittent air flow is delivered to the vest which rapidly expands, compressing the chest wall and producing an oscillation in air flow within the airways. A systematic review ${ }^{167}$ identified a number of studies finding the effectiveness of high-frequency chest wall compression/oscillation to be equal to that of postural drainage and percussion, PEP, oscillating PEP or intrapulmonary percussive ventilation, using a variety of outcome measures. Patient preference for this technique over one oscillating PEP device, or postural drainage and percussion with an assistant, has been reported. ${ }^{190} 191$

\section{Level of evidence 1++}

\section{Recommendation}

- Consider high-frequency chest wall compression/oscillation when recommending an airway clearance technique for adults with cystic fibrosis when stable. (Grade A)

A $2008^{192}$ short-term RCT conducted during hospital admission for an exacerbation found significantly less sputum expectorated when patients used high-frequency chest wall compression/ oscillation in isolation compared with their usual airway clearance technique. There was no associated change in lung function. Of these patients, $55 \%$ preferred their usual airway clearance technique Level of evidence $1+$

Recommendation

- High-frequency chest wall compression/oscillation is not recommended during an infective exacerbation. (Grade B)

\section{Mechanical vibration}

Mechanical vibration is designed to allow independent treatment. Studies tend to combine this technique with postural drainage and cough. ${ }^{193}$ Mechanical vibration was shown to be comparable with conventional physiotherapy in terms of lung function, with patients reporting preference for mechanical vibration. ${ }^{167}$ It is currently not widely available in the UK.

\section{Level of evidence $1++$}

\section{Recommendation}

- Consider mechanical vibration when recommending an airway clearance technique for adults with cystic fibrosis. (Grade A) 
Good practice points

- High-frequency chest wall compression/oscillation, intrapulmonary percussive ventilation and mechanical vibration should be considered where adherence with other airway clearance techniques is problematic.

- Cost implications should be considered when choosing mechanical devices.

\section{Mechanical insufflation-exsufflation/cough assist}

Mechanical in-exsufflation delivers positive pressure followed by negative pressure to the airways, via a mask or mouthpiece. ${ }^{194} \mathrm{~A}$ small-scale observational study assessing the use of mechanical in-exsufflation for adults with $\mathrm{CF}^{195}$ found no increase in peak cough flow in this patient group.

There is insufficient evidence to recommend or refute the use of mechanical in-exsufflation in adult patients with CF.

\section{Level of evidence 3}

Research recommendation

- Further research is required in the use of mechanical insufflation-exsufflation as an airway clearance technique in patients with cystic fibrosis.

\section{Exercise as an airway clearance technique}

The evidence for the use of exercise as an airway clearance technique is unclear. In a systematic review, Main et a ${ }^{167} \mathrm{re}-$ analysed the original data of one study and found a greater improvement in lung function in the conventional physiotherapy group than in the exercise with cycle ergometer group. The study was underpowered and was carried out during an exacerbation of pulmonary infection. In addition, the groups had different mean pulmonary function, and it is difficult to draw definitive conclusions from the results.

Four other studies were omitted from the review as they were of $<7$ days duration. Three of the studies used cycle ergometry and one a circuit of various exercises including walking and cycling. These short-term studies suggest that exercise alone is inferior to other forms of airway clearance with respect to mucus clearance, as measured by sputum weight or radionucleotide labelling. ${ }^{180}{ }^{196-198}$

The addition of exercise to airway clearance techniques leads to enhanced clearance as compared with airway clearance alone ${ }^{180} 196-198$ and is the preferred regimen of many patients. ${ }^{197}$

\section{Level of evidence 4}

Recommendations

- Exercise in isolation should not be used as an airway clearance technique for patients with cystic fibrosis unless adherence to other techniques is problematic. (Grade D)

- The addition of exercise to an appropriate physiotherapy regimen should be considered to increase airway clearance further. (Grade D)

Research recommendation

- Further research is needed to investigate the adjunctive effect and optimal regimen of exercise for enhancing airway clearance in patients with cystic fibrosis.

\section{Naso/oropharangeal suction}

Naso/oropharangeal suction is a procedure intended to remove accumulated secretions from the upper airways and trachea, where cough or other less invasive methods fail. ${ }^{199}$ There are no studies or case reports looking at the use of naso/oropharangeal suction within the CF population.

Suction is rarely used and, where it is, this tends to be for palliation where other secretion reduction methods have failed.

\section{Level of evidence 4}

Recommendation

- Suction should not be considered for use as a routine airway clearance technique in non-intubated patients with cystic fibrosis. (Grade D)

Good practice points

- Suction may be considered during palliative care where all other methods of decreasing secretions have failed and secretions are distressing for the patient.

- Suction may be considered if the patient is unresponsive but secretions are distressing for the relatives or friends present.

\section{Inspiratory muscle training}

The fixed-load method of respiratory muscle training has been developed as an airway clearance technique following studies using it for both assessment of inspiratory muscle function and IMT. During these studies participants reported increased sputum expectoration when repeating the inspiratory manoeuvres. It has been hypothesised that this effect may be similar to that seen with exercise. ${ }^{200}$

Studies of IMT show positive effects on sputum expectoration, lung function, exercise capacity, inspiratory muscle strength and endurance, and anxiety/depression scores. ${ }^{200-202}$ They are, however, short-term studies of low power with some methodological limitations. It is therefore difficult to draw firm conclusions from this evidence. The clinical relevance of improved inspiratory muscle strength/endurance has not been demonstrated.

\section{Level of evidence 2-}

Research recommendations

- Research is needed to support or refute the use of inspiratory muscle training for airway clearance in patients with cystic fibrosis.

- Research is needed to assess the clinical impact of improving inspiratory muscle strength in this patient group.

\section{Non-invasive ventilation}

NIV is a widely accepted technique for hypercapnic respiratory failure or nocturnal hypoventilation and as a bridge to lung transplantation in CF. ${ }^{7203}$ Physiotherapists are often involved in assessment of the patient, set-up of equipment and monitoring of NIV for nocturnal and/or daytime use. ${ }^{76}$

NIV can also be useful as an adjunct to airway clearance techniques in patients with $\mathrm{CF},{ }^{76} 203204$ since it reduces fatigue and dyspnoea ${ }^{204}$ during treatment. MIP, oxygen saturation and small airway function are maintained, ${ }^{204}$ and respiratory rate is lower during treatment with NIV compared with during the active cycle of breathing techniques alone. Moreover, MEP is increased with NIV, with patients reporting easier sputum clearance and a preference for using it..$^{203}$

\section{Level of evidence 1++}

Recommendations

- Trial non-invasive ventilation to assist airway clearance where there is evidence of respiratory muscle weakness or fatigue. (Grade A)

- Trial non-invasive ventilation where desaturation is present during airway clearance. (Grade A)

- Trial non-invasive ventilation to assist airway clearance when the patient has difficulty clearing bronchial secretions with other techniques. (Grade A)

Research recommendation

- Further research is required into the long-term effects of noninvasive ventilation for airway clearance in this patient group. 
NIV may also be used during exercise with the rationale of decreasing dyspnoea, improving oxygenation and thereby improving exercise tolerance, but there is no objective evidence at present to support this.

\section{Research recommendation}

- Further research is required into the short- and long-term effects of non-invasive ventilation during exercise in this patient group.

\section{Intermittent positive pressure breathing}

IPPB provides intermittent pressure ventilation to assist ventilation and augment $V_{\mathrm{T}}$ (see Section 1, COPD). It is used where there is atelectasis or impaired secretion clearance, where there is respiratory muscle weakness or severe hyperinflation, ${ }^{205}$ or when the patient is fatigued. There are no studies of IPPB in the CF population.

Since IPPB is a form of NIV for short-term use, some of the recommendations that apply to NIV are relevant. However, since IPPB concurrently integrates nebulisation, it may have additional effects.

\section{Level of evidence 4}

Recommendation

- Consider a trial of intermittent positive pressure breathing for airway clearance as an alternative to non-invasive ventilation, where the indications for non-invasive ventilation in this situation exist. (Grade D)

Research recommendation

- Further research is required in the use of intermittent positive pressure breathing to aid airway clearance in this patient group.

\section{Continuous positive airway pressure}

Continuous positive airway pressure (CPAP) is predominantly used to correct type 1 respiratory failure that does not respond to oxygen therapy. It uses high-flow oxygen to increase mean airway pressure and improve ventilation to collapsed lung tissue. $^{76}$ There is no published work using CPAP to aid airway clearance in CF. A small short-term trial evaluated the use of CPAP during exercise. ${ }^{206}$ It suggests that in severe CF, exercising with CPAP may decrease oxygen consumption, and increase oxygen saturation and exercise tolerance.

There is insufficient evidence to recommend or refute the use of CPAP to aid either airway clearance or exercise in the CF population.

\section{Level of evidence 2 -}

Research recommendations

- Further research is required in the use of continuous positive airway pressure to aid airway clearance in patients with cystic fibrosis.

- Further research is required in the use of continuous positive airway pressure during exercise in patients with cystic fibrosis.

\section{Inhalation therapies}

The evidence presented here focuses solely on those studies specifically examining the effect on physiotherapy interventions.

\section{Oxygen therapy}

The evidence for the use of oxygen therapy in this client group focuses on its complex practical applications. ${ }^{92} 207208$ The addition of supplemental (ambulatory) oxygen therapy for exercise in advanced disease increases exercise tolerance and aerobic capacity. ${ }^{209}$ Patients require meticulous evaluation and detailed specific prescriptions, ${ }^{92}$ with relevant assessment for ambulatory oxygen. ${ }^{93}$

Physiotherapists should be familiar with current guidance in the delivery of oxygen therapy.

\section{Level of evidence $1++$}

Recommendations

- Administer oxygen therapy, in both the acute and domiciliary settings, according to current national guidance. (Grade A)

- Assess patients with advanced disease for supplemental ambulatory oxygen therapy. (Grade D)

Research recommendation

- Further research into the short- and long-term effects of oxygen therapy during airway clearance and exercise is required.

\section{Humidification}

The upper airway warms, moistens and filters inspired gases unless impaired by pathological processes or bypassed, in which case it is common practice to provide humidification. There is little evidence for the use of humidification in non-intubated patients. ${ }^{92}$ Nebulised saline has been shown to assist sputum clearance in $\mathrm{COPD}^{92}$ and non-CF-related bronchiectasis, ${ }^{210}$ as has nebulised sterile water in non-CF-related bronchiectasis. ${ }^{211}$ Neither has been studied in CF, with the focus rather on hypertonic saline.

\section{Level of evidence 4}

\section{Good practice point}

- Decide on nebulisation-based humidification for the patient with cystic fibrosis on an individual basis.

Research recommendations

- Research into the short- and longer term effects of humidification, particularly with supplemental oxygen, is required.

- Research into the effects of nebulised normal saline on airway clearance in cystic fibrosis is required.

BTS guidelines on emergency oxygen ${ }^{92}$ advise against the use of bubble-through systems, due to lack of evidence of benefit and risk of infection. Large-volume nebulisation-based humidifiers (cold and warm systems) are also identified as a potential infection risk, but may be useful for patients with sputum retention. $^{92}$

\section{Level of evidence $1++$}

Recommendation

- Bubble-through humidification should be avoided due to no evidence of clinical benefit and increased infection risk. (Grade A)

\section{Hypertonic saline}

A Cochrane review ${ }^{212}$ concluded that the inhalation of hypertonic saline significantly improves $\mathrm{FEV}_{1}$ but is less effective than RhDNase (recombinant human DNase) at improving lung function after 3 months of treatment. Studies suggest short-term enhancements in airway clearance with hypertonic saline, as measured by radiolabelled aerosol clearance. Improvements were greater for $12 \%$ than for $3 \%$ solution, and no significant differences were found between $7 \%$ and $12 \%$ concentrations. Improvements are also noted for exercise tolerance and quality of sleep, but predosing with a bronchodilator is important to minimise bronchospasm. ${ }^{213}$ It is an effective, safe and cheap adjunct to physiotherapy, but with insufficient evidence to support its use as a routine treatment for patients with CF. ${ }^{212}$ 


\section{Level of evidence $1++$}

Recommendations

- Consider the addition of hypertonic saline when enhancement of the effectiveness of an airway clearance technique is needed. (Grade A)

- A predose bronchodilator should be used to minimise bronchospasm with inhalation of hypertonic saline. (Grade D)

- A bronchoconstriction trial should be carried out at the initial dose of hypertonic saline to ensure safety and suitability for the patient. (Grade D)

\section{RhDNase}

Two Cochrane reviews ${ }^{212} 214$ assess the effects of RhDNase focusing on lung function, mortality, quality of life, exacerbations, antibiotic use, weight and adverse events as outcome measures. The reviews conclude that inhalation of $\mathrm{RhDNase}$ significantly improves lung function after 1-3 months of treatment.

\section{Level of evidence $1++$}

Recommendation

- RhDNase should be prescribed as per national and local guidelines. (Grade A)

A specific effect of RhDNase on airway/mucociliary clearance has not been assessed and was not used as an outcome measure for the two reviews. It is therefore difficult to assess direct effects of RhDNase on augmenting physiotherapy interventions, but expert opinion would suggest it has a place in aiding removal of secretions.

\section{Level of evidence 4}

\section{Recommendation}

- Consider the use of inhaled RhDNase for enhancing airway clearance effectiveness. (Grade D)

Research recommendation

- Specific research into the effect of inhaled RhDNase on airway clearance is required.

One unpublished study ${ }^{215}$ investigating the effects of inhalation of RhDNase over a 2 -week period suggests it provides greater ventilatory efficiency during submaximal exercise and increased aerobic capacity at maximal exercise.

\section{Level of evidence 3}

\section{Recommendation}

- Consider inhalation therapy with RhDNase for increasing exercise capacity. (Grade D)

\section{Thoracic mobility and strengthening}

Musculoskeletal and postural disorders, common in people with $\mathrm{CF}$, are secondary to pulmonary disease, with a complex relationship between posture and respiration. ${ }^{216-218}$

Thoracic kyphosis and vertebral wedging are more prevalent in people with CF than in healthy controls and are related to worsening lung function and clinical symptoms. ${ }^{216} 218$ There is also evidence of decreased thoracic mobility and muscle weakness compared with matched controls. ${ }^{218}$ These changes in soft tissue and spinal geometry are likely to be attributed to an increased work of breathing and the hunched posture assumed during excessive coughing. The major consequence is a high incidence of back pain, with severity associated with pulmonary deterioration, sputum production and breathlessness. ${ }^{216}$

There is some evidence that spinal deformity is reversible, with the suggestion therefore that the problem may be responsive to therapy. ${ }^{216} \mathrm{~A}$ programme of chest mobility and strengthening exercises to improve posture and coordination was studied over 12 months to determine the effect on lung function. ${ }^{219}$ There was a significant improvement in the slope of $\mathrm{FEV}_{1}$ deterioration, with improved posture, chest wall mobility and body strength. This improvement was combined with a significant reduction in the need for intravenous antibiotics. The numbers in the study were small and the preintervention data were retrospective, but the results highlight the potential for the use of postural interventions in the routine management of patients with CF.

Assessment and appropriate manual therapy treatment of posture correction, chest wall mobility and stretching exercises may decrease the pain and deformity, and prevent progression of these disorders.

\section{Level of evidence 3}

Recommendations

- Question patients with cystic fibrosis about musculoskeletal problems and back pain. (Grade D)

- Assess the problem if present and institute appropriate posture correction, chest wall mobility and stretching exercises or manual therapy treatments where indicated. (Grade D)

\section{Good practice point}

- Effective coughing with appropriate positioning advice should be advocated.

Research recommendation

- Further research is needed to establish the effectiveness of postural correction and exercise in reducing thoracic pain and deformity.

\section{Pelvic floor muscle training}

Urinary incontinence has been shown to be a problem within both the female and male CF population, ${ }^{220}$ with the prevalence of urinary incontinence in women with CF higher than in the normal population. ${ }^{103-106}$

Within this group there may be weak musculature or muscle imbalance, increased intra-abdominal pressure with enlarged liver and persistent cough, in addition to other risk factors common in the non-CF population, for example following pregnancy. Unpublished work $^{102}$ has indicated that there appears to be no problem compared with healthy age-matched controls with the timing of pelvic floor muscle activity or maximal muscle activity (measured by ultrasound imaging and EMG over $1 \mathrm{~s}$ ) during huffing and coughing. EMG activity over $20 \mathrm{~s}$ (representing muscle endurance), however, is reduced during prolonged coughing. ${ }^{102}$

The major cause of urinary incontinence is forced expiration and coughing, thus impacting on the individual's ability and/or willingness to perform airway clearance and lung function manoeuvres, especially during exacerbations of pulmonary infection. The studies noted that symptoms are under-reported, and subjects are unlikely to seek help with the problem. Despite this evidence of prevalence, there are few reports of the assessment and treatment of the problem of urinary incontinence.

One uncontrolled study, ${ }^{221}$ in a self-selected group of women, examined the effect of a 3 -month individualised pelvic floor muscle exercise programme on the strength and endurance of the pelvic muscles. Symptoms were reduced and digital assessment showed significant improvement in muscle endurance. An unpublished study reported improved EMG activity over both 1 and $20 \mathrm{~s}$, and a decrease in symptoms following a more comprehensive programme of pelvic floor muscle exercises and electrical stimulation. ${ }^{107}$ The improvements were main- 
tained at 3 months. Voluntary contraction of the pelvic floor muscles just before and throughout a cough or huff, known as "The Knack", can be used to reduce stress-related leakage of urine. ${ }^{108}$ While there is no robust evidence to advocate physiotherapeutic interventions in the treatment of urinary incontinence in CF, these pilot data lend support to its efficacy, and assessment and treatment of this condition may help prevent or decrease the severity of urinary and/or faecal incontinence.

\section{Level of evidence $2+$}

\section{Recommendations}

- Question patients with cystic fibrosis about their continence status. (Grade D)

- All patients with cystic fibrosis, irrespective of continence status, should be taught to contract the pelvic floor muscles before forced expirations and coughing ("The Knack"). (Grade D)

- If problems of leakage are identified, patients should be referred to a physiotherapist specialising in continence. (Grade D)

- Therapeutic interventions should include an element of endurance training of the pelvic floor muscles to meet the demands of prolonged coughing. (Grade D)

\section{Infection control}

Infection control is of paramount importance in CF because of the wide spectrum of CF pathogens (including various strains of Pseudomonas aeruginosa, Burkholderia cepacia complex, Stenotrophomonas maltophilia and Mycobacterium). Consensus documents have been produced by the Cystic Fibrosis Trust (UK) Infection Control Advisory Group, ${ }^{222}$ and these provide comprehensive guidance based on the available evidence.

\section{Level of evidence $2+$}

\section{Recommendation}

- Physiotherapists caring for patients with cystic fibrosis should be aware of consensus documents regarding infection control. (Grade C)

Good practice points

- Physiotherapists need to be aware of local infection control policies in addition to consensus documents, particularly for the provision of physiotherapy equipment.

- Physiotherapists should help provide guidance on the importance of infection control and equipment maintenance to patients with cystic fibrosis and their carers.

\section{Complementary therapy}

For discussion of the use of these techniques please see Web Appendix 1.

\section{SECTION 4 NON-CYSTIC FIBROSIS-RELATED BRONCHIECTASIS} Introduction

Bronchiectasis refers to the abnormal, irreversible dilatation of the bronchi caused by chronic inflammatory changes in the bronchial walls. ${ }^{223}$ Although most cases of non-CF-related bronchiectasis are idiopathic, recognised causes include childhood respiratory infection, tuberculosis (TB), congenital structural abnormalities, acquired bronchial obstruction, immunodeficiency, primary ciliary dyskinesia (PCD), Young's syndrome, allergic bronchopulmonary aspergillosis (ABPA) and chronic inflammatory disorders (eg, rheumatoid arthritis). ${ }^{224}$

From whatever cause of bronchiectasis, chronic sputum production, air flow obstruction and recurrent respiratory infections are common. ${ }^{224}$ The signs and symptoms of bronchiectasis include chronic cough, purulent sputum, fever, weakness, weight loss, breathlessness (especially on exertion) and fatigue. ${ }^{225}$ A survey in the UK on the physiotherapy management of patients with bronchiectasis found that all physiotherapists use airway clearance techniques, with most respondents also including exercise, ambulation and education on the use of inhaled therapy. ${ }^{226}$

Exercise, pulmonary rehabilitation and inspiratory muscle training Reduced exercise tolerance can be a problem for some individuals with bronchiectasis; those with reduced exercise capacity and expiratory flow limitation have higher Medical Research Council (MRC) dyspnoea scores. ${ }^{227}$ There is little research on physical exercise or IMT in the non-CF-related bronchiectasis population. A 2002 Cochrane review ${ }^{228}$ concluded from the limited data available (two abstracts) that IMT improved endurance exercise capacity and health-related quality of life.

A more recent study ${ }^{229}$ investigated the effects of an 8-week high-intensity pulmonary rehabilitation (PR) programme and IMT on stable patients with non-CF-related bronchiectasis. Patients $(n=32)$ were randomly allocated to one of three groups: PR plus IMT; PR plus sham IMT; and a control group. Both PR groups gained significant increases in both maximal and endurance exercise capacity, and inspiratory muscle strength, compared with the controls. There were no statistically significant differences in the improvements between these two groups. Three months following the training programme, the improvement in exercise capacity was maintained only in the group which included IMT. IMT, simultaneously with PR, had no short-term additional benefit, but appeared to have a positive effect on the maintenance of the training effect.

Current guidance on PR recommends it for all patients with chronic respiratory disease. ${ }^{1446}$

\section{Level of evidence 1+}

Recommendations

- Offer pulmonary rehabilitation to individuals with noncystic fibrosis-related bronchiectasis who have breathlessness affecting their activities of daily living. (Grade A)

- Consider the use of inspiratory muscle training in conjunction with conventional pulmonary rehabilitation to enhance the maintenance of the training effect. (Grade B)

\section{Airway clearance}

There is considerably less evidence on the use of airway clearance techniques in non-CF-related bronchiectasis than in CF patients. Extrapolation of findings is inevitable, but should be done with caution, since the properties of sputum in these two conditions may vary considerably.

A small $(n=8)$ short-term study demonstrated an increase in sputum yield for physiotherapy compared with no physiotherapy in non-CF-related bronchiectasis. ${ }^{230}$ This study measured sputum yield, however only during and $30 \mathrm{~min}$ after the treatment period. An earlier single intervention study ${ }^{231}$ on a mixed population $(n=6)$ found that physiotherapy (postural drainage, shaking, vibrations, percussion and coughing) (see Glossary, Appendix A) increased sputum yield and peripheral lung clearance compared with cough alone. The authors concluded that chest physiotherapy is necessary for the enhancement of impaired peripheral lung clearance.

There is no published literature on the long-term effects of physiotherapy for airway clearance in this patient group. A Cochrane review ${ }^{232}$ highlights the need for adequately sized, 
high-quality RCTs performed on subjects with uniform pulmonary conditions which test the effects of a course of a treatment rather than a single treatment.

Despite the lack of published evidence, it is widely accepted clinical practice that airway clearance is important in the management of people with a chronic, productive cough and/or evidence of mucus plugging on CT, to enhance sputum clearance and reduce cough frequency. A study evaluating serial CT changes in people with non-CF-related bronchiectasis found that sputum plugging was associated with deterioration in pulmonary function and oxygenation. ${ }^{233}$ Review of the CT scan identifies the affected bronchopulmonary segments, and a targeted, regular, effective airway clearance technique may help to prevent or delay clinical deterioration.

For individuals with a non-productive cough, it is even less clear, but expert opinion advocates teaching an airway clearance technique to ensure there is no sputum retention and also to use during exacerbations of pulmonary infection. Consensus of expert opinion, however, is that these patients do not benefit from daily airway clearance technique use.

As with other chronic conditions, it is considered good practice and is usually patient preference, to use a technique that allows independent treatment where possible.

\section{Level of evidence 4}

Recommendation

- Teach all patients with bronchiectasis and a chronic, productive cough, and/or evidence of mucus plugging on CT, an airway clearance technique for use as necessary. (Grade D)

\section{Good practice points}

- If available, use the CT scan to identify affected bronchopulmonary segments to facilitate effective treatment.

- Use an airway clearance technique that allows independent treatment where possible.

- Teach individuals with a non-productive cough an appropriate airway clearance technique to use during exacerbations of pulmonary infection.

\section{Frequency and duration of treatment}

Although there is a lack of evidence, pragmatically, frequency and duration of treatment are related to sputum volume, lifestyle and diurnal variation of the patient's sputum production.

For patients who are moderately productive, it is generally considered sufficient to carry out airway clearance twice daily, and once a day for patients who are less productive. During an infective exacerbation, it is usually advised to add in an extra session. In those who are extremely productive of sputum it is important to find a balance between treatment sessions being of adequate length to maximise airway clearance, but not so long that the patient becomes fatigued.

Within 3 months of an initial appointment, the individual's ability to carry out the designated technique effectively should be reviewed, ${ }^{234}$ along with the optimal frequency and duration of treatment, to ensure patient satisfaction. Follow-up should be based on efficacy of the demonstrated technique, the patient's understanding and disease severity.

The patient should be made aware of other available airway clearance technique options, as patient preference for treatment must be taken into account and this also may enhance adherence to treatment.

\section{Level of evidence 4}

\section{Recommendations}

- Review the effectiveness and acceptability of the chosen airway clearance technique within approximately 3 months of the initial visit. (Grade D)
- Patients should be made aware of other available airway clearance technique options. (Grade D)

Good practice points

- The frequency and duration of the airway clearance technique should be specific to the needs of the individual patient, which may alter with periods of infective exacerbation.

- Where possible, the treatment session should continue until most of the excess secretions are expectorated but should not be so long that the patient becomes fatigued.

\section{Airway clearance techniques}

There are a variety of airway clearance techniques that can be used, although a survey in the UK on the physiotherapy management of patients with bronchiectasis found that $91 \%$ of physiotherapists taught the active cycle of breathing techniques routinely. ${ }^{226}$ Other techniques such as PEP, oscillating PEP, autogenic drainage and IPPB were used less frequently (see Glossary, Appendix A for all terms). Most respondents also included exercise, ambulation and education on the use of inhaled therapy in the management of this patient group. Treatment choice appeared to be influenced as much by clinical and local experience as by published studies, reflecting the limited evidence in this area, with $87 \%$ of respondents highlighting a need for further research.

\section{Postural drainage (gravity-assisted positioning)}

The effectiveness of postural drainage on clearing sputum in the non-CF-related bronchiectasis patient was graphically described in $1901,{ }^{3}$ and the lower viscosity of the sputum, compared with that of the CF population, may lend itself more readily to the continued use of postural drainage today.

A small single intervention $(n=10)$ study $^{235}$ on a mixed population found that the addition of postural drainage to the FET (see Glossary, Appendix A) resulted in a significantly greater sputum yield than the FET alone.

In a 2007 three-way crossover RCT $(n=36),{ }^{236}$ a single treatment of the active cycle of breathing techniques in a postural drainage position was compared with a treatment performed in sitting and with one oscillating PEP device, also used in sitting. The treatment with postural drainage yielded a sputum wet weight twice that of either technique in sitting.

Although it could therefore be reasonably concluded that postural drainage is the key component to effective sputum clearance, at least in the short term, the treatment with postural drainage was associated with significantly more discomfort than the treatments in sitting and was felt to interfere more with daily life than the oscillating PEP. Preference for the oscillating PEP was rated by $44 \%$ of subjects, for the active cycle of breathing techniques in sitting by $22 \%$ and for the active cycle of breathing techniques in a postural drainage position by $33 \%$. Moreover, although there was no significant difference in treatment duration among the three interventions, active cycle of breathing techniques plus postural drainage was perceived by subjects as being significantly more time consuming. It should be noted that a single intervention may not reflect the longer term outcome.

\section{Level of evidence 1+}

Recommendations

- Where it is found to enhance airway clearance and has no unwanted side effects, postural drainage should be taught and encouraged. (Grade B)

- Patient preference and adherence to treatment must be taken into account. (Grade B) 
Good practice point

- Assess the effectiveness of the position on airway clearance.

An old (1986) questionnaire survey $(n=50)$ on a mixed population of subjects with hypersecretory pulmonary disease $e^{237}$ found that $46 / 50$ (92\%) subjects found postural drainage helpful during exacerbations of their chest symptoms. Thirty-five of the 50 subjects (70\%) found postural drainage also helpful between exacerbations. This latter group produced significantly more sputum daily between exacerbations compared with the remainder of the subjects.

There is no current evidence, however, to confirm or refute the addition of postural drainage in the long-term management of airway clearance for this client group.

Research recommendation

- Research is required into the long-term effects of techniques incorporating postural drainage compared with those that do not.

The CT scan will help identify affected bronchopulmonary segments and aid selection of the appropriate postural drainage position(s). Postural drainage positions for the mid and basal zones of the lung require a head-down tilt, and contraindications and precautions to this posture can be found in physiotherapy textbooks. ${ }^{36}$

The presence of gastro-oesophageal reflux (GOR) should be taken into consideration when using a head-down tilt. Chen et $a l,{ }^{238}$ however, did not find the frequency or duration of GOR significantly affected by postural drainage in individuals with chronic bronchitis and bronchiectasis. This was true of individuals both with and without GOR.

The head-down tilt may be problematic for the breathless patient, in particular the extreme tilts required for the basal areas, including the Trendelenburg position (see Glossary, Appendix A). In the CF population, the use of non-invasive ventilatory support has been shown to allow the patient with advanced disease $e^{203}$ to tolerate postural drainage positions better that otherwise make them too breathless. It is reasonable to extrapolate these findings to the non-CF-related bronchiectasis population.

\section{Level of evidence 4}

Recommendations

- Take comorbidities, and contraindications and precautions to head-down tilt positions into account. (Grade D)

- Consider offsetting the increased load of breathing in a breathless patient by the use of non-invasive ventilation or intermittent positive pressure breathing where postural drainage is essential for clearing secretions. (Grade D)

Good practice point

- Use the CT scan to aid selection of postural drainage positions.

\section{Modified postural drainage}

Cecins et $a^{187}$ compared sputum yield for the active cycle of breathing techniques, in both a horizontal and a postural drainage position with a head-down tilt in 19 subjects with bronchiectasis. All subjects produced $>20 \mathrm{~g}$ of sputum per day. Although there was no significant difference between the two treatments in terms of wet weight of sputum expectorated, 18 of the 19 subjects preferred the horizontal position. This may be important when considering long-term adherence to treatment. However, these results must be interpreted with caution, since only five of the subjects had non-CF-related bronchiectasis.

Modified postural drainage positions with no head-down tilt are often better tolerated and may be as effective as tipped positions, but further research is required to verify this in the non-CF-related bronchiectasis population.

\section{Level of evidence 1 -}

Good practice points

- Offer modified postural drainage positions (no head-down tilt) as an alternative only if as effective as the correct postural drainage position.

- Offer modified postural drainage positions (no head-down tilt) as an alternative if using the correct postural drainage position is problematic for the patient in any way.

Research recommendation

- Further research is required on the efficacy of modified postural drainage in patients with non-cystic fibrosis-related bronchiectasis.

\section{Manual techniques}

Manual techniques (chest wall percussion and thoracic cage compression, with or without shaking or vibrations) are used by physiotherapists with the aim of enhancing airway clearance. They are most typically used in the UK in conjunction with the active cycle of breathing techniques; more commonly when the patient is unwell or fatigued and needing help with their usual regimen. There is scant evidence on the effectiveness of manual techniques in the clearance of secretions compared with independent techniques.

\section{Chest wall percussion}

Chest wall percussion may be fast or slow and single- or doublehanded. In one small controlled cohort crossover trial, the addition of all types of chest percussion in random order to postural drainage and the FET, in patients with copious sputum production, ${ }^{239}$ significantly increased the rate, but not the volume, of sputum production, compared with no percussion. The order of effect was greatest for fast double-handed percussion and least for slow single-handed percussion. The results must be interpreted with caution, since the study was small, had a mixed population and only one therapist applied all the techniques. Another small mixed population study $(n=8)^{240}$ found that chest wall percussion plus postural drainage and deep breathing exercises significantly increased dry sputum weight expectorated compared with postural drainage alone. Chest percussion plus postural drainage with tidal breathing was less effective. Tracheobronchial clearance (measured by labelling bronchial secretions with an inhaled radioaerosol), however, was unaffected by the addition of chest percussion with or without deep breathing. Chest wall percussion, when used with postural drainage, does not adversely affect oxygen saturation, ${ }^{239} 241$ heart rate ${ }^{241}$ or pulmonary function $^{239}$ in non-CF-related bronchiectasis.

There is insufficient evidence to support or refute the use of chest wall percussion in this population.

\section{Level of evidence 2 -}

\section{Chest wall vibration}

A small mixed population study $(n=8)^{240}$ found that chest wall vibratory shaking, plus postural drainage and deep breathing exercises, significantly increased sputum weight expectorated compared with postural drainage alone, but had no effect on tracheobronchial clearance (measured by radiolabelled aerosol). In a within-subject, experimental study in three healthy subjects, ${ }^{189}$ seven experienced cardiopulmonary physiotherapists applied various manual treatments in random order during passive expiration: vibration (chest compression with oscillation), compression alone and oscillation alone. Change in 
intrapleural pressure was compared with that with passive expiration alone. During vibration, $13 \%$ of the change in intrapleural pressure was attributed to compression, $12 \%$ to oscillation and $75 \%$ to lung recoil. They concluded that changes in intrapleural pressure occurring during vibration appear to be the sum of changes from lung recoil and the components of the technique, suggesting that all three factors are required to optimise expiratory flow. Thoracic cage compression as part of autogenic drainage during the expiratory phase can be used to facilitate lower lung volume level breathing and provides feedback for both the patient and physiotherapist.

There is insufficient evidence to support or refute the use of chest wall vibrations or shaking in non-CF-related bronchiectasis

\section{Level of evidence 2-}

Good practice points

- Consider manual techniques when patients using independent techniques are unable to clear secretions effectively.

- Offer manual techniques as part of an airway clearance regimen during an acute exacerbation, or when the patient is more fatigued than usual.

Research recommendation

- Further research is required into whether manual techniques enhance the efficacy of independent airway clearance in patients with non-cystic fibrosis-related bronchiectasis.

\section{Active cycle of breathing techniques}

The active cycle of breathing techniques is the most commonly used airway clearance technique in the $\mathrm{UK}^{226}$ and is frequently used in conjunction with postural drainage, and both with and without manual techniques. It incorporates the FET (see Glossary, Appendix A).

In a 4-week, randomised, crossover trial ${ }^{242}$ weight of sputum expectorated was similar with the active cycle of breathing techniques and one oscillating PEP device, used with the FET and with both techniques performed in postural drainage positions as deemed necessary. An RCT ${ }^{236}$ found no significant difference in sputum wet weight between a single treatment of the active cycle of breathing techniques and the oscillating PEP in sitting, but neither was as effective as the active cycle of breathing techniques with postural drainage. Forty-four percent of subjects rated their preference for oscillating PEP, $22 \%$ for active cycle of breathing techniques in sitting and 33\% for active cycle of breathing techniques in a postural drainage position. When combined with postural drainage, however, the active cycle of breathing techniques was associated with significantly more discomfort and was felt to interfere more with daily life than the oscillating PEP; it was also perceived as being significantly more time consuming than both techniques in sitting.

A randomised, crossover, single intervention study ${ }^{211}$ found no significant difference in weight of sputum expectorated when comparing active cycle of breathing techniques plus manual techniques with a different oscillating PEP device (used with the FET), with both techniques performed in postural drainage positions. The test of incremental respiratory endurance (TIRE) was significantly less effective than the active cycle of breathing techniques, used in conjunction with postural drainage and manual techniques. ${ }^{243}$

\section{Level of evidence $1+$}

\section{Recommendation}

- Consider the active cycle of breathing techniques when recommending an airway clearance technique for adults with non-cystic fibrosis-related bronchiectasis. (Grade A)

\section{Autogenic drainage}

A sole pilot study $(n=13)$ compared the effects of a single session of autogenic drainage with a control of no physiotherapy) ${ }^{244}$ on sputum weight and a measure of airway resistance, the interrupter technique (Rint). Significantly more sputum was produced during the autogenic drainage session compared with the control. No changes in airways resistance were found; however, the absence of a significant change following the autogenic drainage could be because either airways resistance does not alter or the interrupter technique is not sensitive enough to detect changes in the airways of adults with bronchiectasis. There is insufficient evidence to support or refute the use of autogenic drainage in this population.

\section{Level of evidence 1 -}

Good practice point

- Autogenic drainage may be offered as an alternative airway clearance technique if other techniques are less effective or acceptable to the patient with non-cystic fibrosis-related bronchiectasis.

Research recommendations

- Further research is required to assess the effectiveness of autogenic drainage in adults with non-cystic fibrosis-related bronchiectasis.

- Further research is required to establish whether the interrupter technique is a valid outcome measure for use in adults with bronchiectasis.

\section{Positive expiratory pressure}

There is little published evidence on the use of PEP in the nonCF-related bronchiectasis population. A small, single intervention pilot study $(n=8)$ studied the ciliary and cough transportability of non-CF-related bronchiectasis sputum after using PEP. ${ }^{245}$ Sputum transportability was not altered postintervention. However, the authors note that the sample size was extremely small and the outcome measurement of relative velocity may not have been sensitive to alterations in sputum viscoelasticity.

\section{Level of evidence 2-}

Good practice point

- Positive expiratory pressure may be offered as an alternative airway clearance technique if other techniques are less effective or acceptable to the patient with non-cystic fibrosis-related bronchiectasis.

Research recommendation

- Further research is required to assess the effectiveness of positive expiratory pressure in adults with non-cystic fibrosis-related bronchiectasis.

\section{Oscillating positive expiratory pressure}

The same small single intervention pilot study ${ }^{245}(n=8)$ also found no difference in transportability of non-CF-related bronchiectasis sputum after using an oscillating PEP device. Conversely, a single abstract reports that two oscillating PEP devices produce a significant reduction of bronchiectasis sputum cohesiveness in vitro at $30 \mathrm{~min} .^{246}$

In a 4-week crossover trial, ${ }^{242}$ twice-daily treatment with one oscillating PEP device, used with the FET and postural drainage, was as effective as the active cycle of breathing techniques and postural drainage for median weekly sputum weight. Neither technique had an adverse effect on PEFR or breathlessness. Eleven of the 17 subjects expressed a preference for the oscillating PEP device. A single intervention study found no significant difference between one oscillating PEP device (used 
in sitting) and the active cycle of breathing techniques (performed in sitting) for mean difference in sputum wet weight, ${ }^{236}$ but neither was as effective as the active cycle of breathing techniques with postural drainage. Although there was no statistically significant difference in preference for interventions, $44 \%$ of subjects preferred the oscillating PEP and found it interfered less with daily life.

In a single intervention trial using stable subjects, ${ }^{211}$ an alternative oscillating PEP device (used with postural drainage and the FET) has been shown to be as effective as the active cycle of breathing techniques (used with postural drainage, both with and without manual techniques) as measured by wet sputum weight, spirometry, $\mathrm{SpO}_{2}$, breathlessness and treatment duration. Although not statistically significant, a greater proportion of subjects (14/20) preferred the oscillating PEP device. The authors felt this preference might have been due to the short-term novelty factor or due to the fact that the subjects were able to carry out treatment independently.

In a pilot study in Hong Kong, ${ }^{247} 15$ patients with an acute exacerbation of bronchiectasis were randomly allocated to three groups: one oscillating PEP device plus deep breathing and coughing, breathing and coughing plus postural drainage, and breathing and coughing alone. There were no differences between groups in sputum production or lung function measurements. Patients reported that all techniques were equally easy to use, but the oscillating PEP device was perceived as being the most effective.

\section{Level of evidence 1+}

\section{Recommendation}

- Consider oscillating positive expiratory pressure when recommending an airway clearance technique for adults with non-cystic fibrosis-related bronchiectasis. (Grade A)

\section{Test of incremental respiratory endurance}

The TIRE has been proposed as a method of airway clearance in bronchiectasis. ${ }^{243}$ A randomised crossover study compared a single session of the active cycle of breathing techniques (including postural drainage and vibrations) with a single session of the TIRE in 20 patients with stable bronchiectasis. Sputum weight, expectorated during and $30 \mathrm{~min}$ following treatment, was significantly greater with the active cycle of breathing techniques, postural drainage and vibrations.

\section{Level of evidence 1+}

Recommendation

- The test of incremental respiratory endurance should not be considered as a first-line airway clearance technique. (Grade B)

\section{Combinations of airway clearance techniques}

The combination of techniques used in these studies makes interpretation difficult, but it would seem that the active cycle of breathing techniques and oscillating PEP devices are equally effective when used in conjunction with the FET and postural drainage. The only exception to this general finding was the Hong Kong study, ${ }^{247}$ which used slightly different treatment regimens.

The FET can be used with all the aforementioned airway clearance techniques and is an integral part of the active cycle of breathing techniques. To add somewhat to the confusion, some of the early studies of the FET probably referred to the active cycle of breathing techniques, since the treatment evaluated included deep breathing. ${ }^{169} 235$ FET, despite producing a significantly lower expiratory flow, was found to be as effective as coughing in the clearance of an inhaled, deposited radioaerosol from the inner and intermediate regions of the lungs. ${ }^{248}$ An earlier study, ${ }^{235}$ again on a mixed population $(n=10)$, found that the FET yielded significantly more sputum than directed coughing. However, sputum yield obtained by the FET, when used in combination with postural drainage, was significantly greater than the FET alone.

Extrapolating from all these studies, the inclusion of both the FET and postural drainage in most regimens for the patient with non-CF-related bronchiectasis appears to increase efficacy.

\section{Level of evidence $1+$}

Recommendations

- The inclusion of postural drainage should be considered for all airway clearance techniques. (Grade B)

- The inclusion of the forced expiration technique should be considered for all airway clearance techniques. (Grade B)

Research recommendation

- Further research is needed to investigate the relative efficacy of different airway clearance techniques in non-cystic fibrosis-related bronchiectasis.

\section{Adjuncts to airway clearance}

There are a number of adjuncts that may be used to enhance the effectiveness of an airway clearance technique.

\section{Humidification}

Humidification can be used as an adjunct to airway clearance. It is thought that humidification enhances ciliary function and increases the efficiency of the cough mechanism. ${ }^{249}$ A small study $(n=7)$ showed that 30 min of cold water, jet nebulising humidification via a facemask before physiotherapy (postural drainage and FET) significantly increased sputum yield and radioaerosol clearance, compared with physiotherapy alone, in non-CF-related bronchiectasis. ${ }^{249}$

\section{Level of evidence 1+}

\section{Recommendation}

- Consider nebulised sterile water inhalation before treatment to enhance sputum clearance (Grade B)

\section{Nebulised $\beta_{2}$-agonists}

In two four-way randomised crossover trials (17 years apart), the use of $5 \mathrm{mg}$ of nebulised terbutaline immediately prior to physiotherapy (FET plus postural drainage, ${ }^{230}$ or the active cycle of breathing techniques plus modified postural drainage ${ }^{210}$ ) yielded significantly more sputum ${ }^{210} 230$ and increased radiolabelled aerosol clearance from the lung ${ }^{230}$ compared with physiotherapy alone. Nebulised terbutaline may enhance sputum yield as a result of direct hydration and/or $\beta_{2}$-adrenergic stimulation. ${ }^{210}$ In addition, the ensuing bronchodilation may enhance airway clearance by increasing expiratory flow rates and/or improving regional ventilation. ${ }^{210}$

\section{Level of evidence $1+$}

\section{Recommendation}

- Consider nebulised $\beta_{2}$-agonists before treatment to enhance sputum clearance. (Grade B)

\section{Nebulised hypertonic saline}

Sodium and chloride concentrations in non-CF-related bronchiectasis sputum are below those found in plasma and, if the saline concentration of sputum is reduced, viscosity and elasticity are increased, which will impair ciliary transportability. ${ }^{250}$ Nebulised hypertonic saline (commonly 7\%) is thought to work by increasing sputum salinity, thereby altering 
its rheology so that it is cleared more easily by the cilia. ${ }^{224}$ In 2005 , Kellett et $a^{210}$ randomised clinically stable subjects with low sputum yield $(<10 \mathrm{~g}$ sputum per day) to four single treatments of the active cycle of breathing techniques (in a modified postural drainage position), as follows: alone, or preceded by (1) nebulised terbutaline; (2) nebulised terbutaline and nebulised normal saline (0.9\%); or (3) nebulised terbutaline and nebulised hypertonic saline (7\%). Sputum yield, viscosity and ease of sputum expectoration were improved by all nebulised treatments, in ascending order.

The study included a hypertonic saline challenge test on each subject, since it has been found to decrease $\mathrm{FEV}_{1}$ in people with asthma ${ }^{251}$ and $\mathrm{CF}^{252}$ and a test dose is considered necessary. ${ }^{234}$ None of the subjects demonstrated evidence of bronchoconstriction, although it should be noted that subjects with allergic bronchopulmonary aspergillosis and cystic fibrosis phenotypes were excluded from the study ${ }^{210}$ and all subjects received nebulised terbutaline prior to the nebulised hypertonic saline dose. Pretreatment with a bronchodilator may be particularly necessary for those with bronchial hyper-reactivity. ${ }^{253}$

\section{Level of evidence $1+$}

Recommendations

- Consider nebulised hypertonic saline before airway clearance to increase sputum yield, reduce sputum viscosity and improve ease of expectoration. (Grade B)

- When first administered, $\mathrm{FEV}_{1}$ or peak expiratory flow rate should be measured before and after nebulised hypertonic saline to assess for possible bronchoconstriction. (Grade D)

- Pretreat with a bronchodilator, particularly for those with bronchial hyper-reactivity. (Grade D)

Research recommendations

- Research is required to determine the long-term effects of hypertonic saline.

- Research is required to determine effectiveness in patients who produce $>10$ g sputum per day.

\section{Nebulised normal saline}

In the study of Kellet et al described above, ${ }^{210}$ the active cycle of breathing techniques (in a modified postural drainage position), preceded by nebulised terbutaline and nebulised normal saline $(0.9 \%)$, resulted in significantly greater sputum weight and a greater reduction in sputum viscosity than the active cycle of breathing techniques (in a modified postural drainage position), either alone or preceded by nebulised terbutaline. However, the improvement was less than when the active cycle of breathing techniques (in a modified postural drainage position) was preceded by nebulised terbutaline and nebulised hypertonic $(7 \%)$ saline. A smaller $(n=8)$, earlier (1988) study ${ }^{230}$ also found that the addition of nebulised normal saline immediately prior to physiotherapy (FET and postural drainage) yielded significantly more sputum than physiotherapy alone.

\section{Level of evidence $1+$}

\section{Recommendation}

- Consider nebulised normal saline before airway clearance to increase sputum yield, reduce sputum viscosity and improve ease of expectoration when hypertonic saline is not suitable or available. (Grade B)

\section{Non-invasive ventilation and intermittent positive pressure breathing}

There is no published evidence available for the use of NIV or IPPB in association with physiotherapy in the non-CF-related bronchiectasis population, but clinical trials in other conditions and expert opinion supports its use in this situation.
NIV, including IPPB, provides positive pressure throughout inspiration, augmenting tidal volume $e^{76-79} 86-89$ and decreasing the work of breathing ${ }^{85}$ in COPD patients, if the machine is set up correctly. It is postulated that this assistance to inspiration enhances the effect of the deep breathing part of an airway clearance technique and allows the fatigued patient better to tolerate and carry out airway clearance, which might otherwise be too tiring.

In the CF population, NIV has been demonstrated to be useful as an adjunct to airway clearance techniques, ${ }^{203} 204$ since it reduces fatigue and dyspnoea ${ }^{204}$ during treatment. MIP, oxygen saturation and small airway function are maintained, ${ }^{204}$ and respiratory rate is lower, during treatment with NIV. Moreover, patients have increased MEP, and report easier sputum clearance and a preference for using it. ${ }^{203}$ NIV allows the patient with advanced disease to tolerate longer periods of physiotherapy and to adopt postural drainage positions that would otherwise make them too breathless. ${ }^{19}$

It is reasonable to extrapolate these findings to the non-CFrelated bronchiectasis population.

\section{Level of evidence 4}

\section{Recommendation}

- Consider non-invasive ventilation or intermittent positive pressure breathing to augment tidal volume and reduce the work of breathing in patients who are becoming fatigued and finding airway clearance difficult. (Grade D)

\section{Pelvic floor muscle training}

The degree of urinary incontinence has been shown to be greater in those with chronic cough due to COPD and CF, compared with a normal population with stress incontinence. ${ }^{102}$ Evidence of increased prevalence of stress incontinence, compared with a normal population, as yet exists only for patients with CF. ${ }^{103-106}$

The main factor thought to influence this, chronic cough, is present in those with non-CF-related bronchiectasis in addition to risk factors common in the general population, for example following pregnancy. Symptoms are underreported, and subjects are unlikely to seek help with the problem. The presence of urinary incontinence may impact on the individual's ability and/or willingness to perform certain activities, such as some exercises, or airway clearance and lung function manoeuvres, especially during exacerbations of pulmonary infection.

Although there is no evidence in this population, intervention by a specialist continence physiotherapist in those with COPD and CF produced positive effects. ${ }^{107}$ Voluntary contraction of the pelvic floor muscles just before and throughout a cough or huff, known as "The Knack", has been used successfully to reduce stress-related leakage of urine in a general population. ${ }^{108}$ It is reasonable to extrapolate these findings to the non-CFrelated bronchiectasis population.

\section{Level of evidence 4}

Recommendations

- Patients should be questioned about their continence status. (Grade D)

- All patients with chronic cough, irrespective of continence status, should be taught to contract the pelvic floor muscles before forced expirations and coughing ("The Knack"). (Grade D)

- If problems of leakage are identified, patients should be referred to a physiotherapist specialising in continence. (Grade D) 


\section{Complementary therapy}

For discussion of the use of these techniques please see Web Appendix 1.

\section{SECTION 5 RESTRICTIVE LUNG CONDITIONS}

\section{Introduction}

This section covers commonly seen restrictive lung conditions, such as pneumonias and pulmonary fibrosis, including that following TB. These conditions are characterised by reduced lung volumes (residual volume (RV), total lung capacity (TLC) and functional residual capacity (FRC)) and can be either intrinsic (disease of the lung parenchyma) or extrinsic (extraparenchymal disease). Although there are other conditions within this category, no evidence could be found relating to physiotherapeutic techniques. Restrictive disorders arising from chest wall and neuromuscular conditions are covered in Section 6 within this guideline.

Dry cough, progressive dyspnoea and loss of exercise tolerance and function are frequently occurring symptoms in restrictive lung conditions potentially amenable to physiotherapy. Optimisation of oxygen therapy, delivery of appropriate non-invasive ventilatory support, management of breathlessness, exercise, advice and maximisation of functional activity are aspects of care that physiotherapists provide. In the chronic setting, only the effects of pulmonary rehabilitation have been studied in patients with lung fibrosis. In the acute setting, the scant research there is has been undertaken only in patients with uncomplicated community-acquired pneumonia.

\section{Lung fibrosis}

\section{Pulmonary rehabilitation}

The rationale for $\mathrm{PR}$ remains the same in non-COPD as for COPD, although there is little specific published evidence on the effectiveness of PR for patients with restrictive lung disease. In the few available studies, however, the results for non-COPD patients are as good ${ }^{254-256}$ or better ${ }^{257} 258$ than for COPD. In one of the small studies in patients with interstitial lung fibrosis demonstrating statistically and clinically important benefits from PR, which exceeded those seen in $\mathrm{COPD},{ }^{258}$ the authors postulate that the large improvements seen in the restrictive lung disease group were perhaps attributable to the PR taking place early in the course of the disease. All current guidance recommends including nonCOPD patients in PR and that the content of education sessions should be adjusted accordingly. ${ }^{14} 46$

\section{Level of evidence $2++$}

Recommendation

- All patients with chronic restrictive conditions, such as pulmonary fibrosis, should be considered for pulmonary rehabilitation. (Grade B)

Good practice points

- Patients with restrictive lung disease should be referred for pulmonary rehabilitation as early as possible in the disease process.

- The content of education sessions should be adjusted accordingly.

Research recommendation

- Research is required into the effects of physiotherapeutic strategies for the management of dyspnoea and cough in this patient group.

\section{Acute pneumonia}

\section{Mobilisation during hospital admission}

In a large study of 458 patients with community-acquired pneumonia, ${ }^{259}$ length of stay in hospital, chest $x$ ray changes and mortality were compared between a control group (usual treatment) and an early mobilisation group. This consisted of sitting out of bed for at least 20 min within the first $24 \mathrm{~h}$ of hospital admission, with increasing mobility on subsequent days. A significant reduction in length of stay was found in the early mobilisation group compared with the usual care group.

\section{Level of evidence $1+$}

\section{Recommendation}

- Medical condition permitting, patients admitted to hospital with uncomplicated community-acquired pneumonia should sit out of bed for at least 20 min within the first $24 \mathrm{~h}$ and increase mobility each subsequent day of hospitalisation. (Grade B)

\section{Airway clearance techniques}

In this client group, only the traditional techniques to facilitate removal of airway secretions (breathing exercises, postural drainage, percussion and vibrations) have been studied. It should be noted that these studies were conducted in patients with uncomplicated pneumonia and did not include patients with existing COPD or other chronic respiratory disease. Furthermore, practice today rarely includes formal airway clearance techniques for pneumonia, unless there is copious sputum production or difficulty expectorating, with the current emphasis on mobilisation and restoration of function, and correction of respiratory or ventilatory failure.

Patients with primary pneumonia $(n=171)$ were randomised $^{260}$ to receive either daily airway clearance techniques (consisting of postural drainage, external help with breathing, percussion and vibration for 15-20 min) or advice on the need for expectoration and deep breathing only. There were no significant differences in length of stay, healing time and $\mathrm{FEV}_{1}$. There was, however, a significant increase in the duration of fever in patients who had received the first regimen. Further analysis revealed significant increases in length of stay and fever in patients under the age of 47 years, in those who smoked, or in those with mainly interstitial, as opposed to alveolar, infiltrates. They did note that there might be benefit in advising patients verbally about the importance of active expectoration.

Tydeman ${ }^{261}$ compared no physiotherapy with twice-daily physiotherapy consisting of breathing control and localised expansion exercises and, where there was production of sputum, postural drainage, percussion, vibration and thoracic expansion exercises. There were no significant differences between the two groups in terms of FVC, length of stay, sputum weight, duration of antibiotic therapy and visual analogue scores for well-being and breathlessness.

\section{Level of evidence $1+$}

Recommendation

- Patients admitted with primary uncomplicated pneumonia should not be treated with traditional airway clearance techniques routinely. (Grade B)

Good practice points

- Patients should be offered advice regarding expectoration if sputum is present.

- Consider airway clearance techniques if the patient has sputum and difficulty with expectoration or in the event of a pre-existing lung condition.

\section{Positive expiratory pressure}

Although there are no studies of the more usual form of PEP, one large Scandinavian triall ${ }^{262}$ used "bottle blowing", a simple form of PEP (bubble PEP; see Glossary, Appendix A). Patients 
admitted with uncomplicated pneumonia were randomised into three groups. All groups received early mobilisation and instruction to "cough by huffing", the control treatment (Group A). Group B were also instructed to sit up and take 20 deep breaths every hour throughout the day. Group $\mathrm{C}$ were given bubble PEP in addition and were directed to blow at a "calm speed", 20 times every hour for 10 consecutive hours each day. Although there were no differences in C-reactive protein (CRP), erythrocyte sedimentation rate (ESR), $\mathrm{FEV}_{1}$, peak expiratory flow (PEF) or vital capacity (VC), there was a significant reduction in length of stay for Group $\mathrm{C}$ (bottle blowing) compared with A (control), with a non-significant trend for reduction in fever.

\section{Level of evidence $1+$}

\section{Recommendation}

- In patients with uncomplicated community-acquired pneumonia admitted to hospital, the regular use of positive expiratory pressure should be considered. (Grade B)

\section{Continuous positive airway pressure}

CPAP can improve oxygenation in patients with diffuse pneumonia who remain hypoxaemic despite maximal medical treatment, ${ }^{263} 264$ and reduce respiratory rate and breathlessness in patients with Pneumocystis carinii pneumonia. ${ }^{264}$ Current BTS guidance ${ }^{76}$ recommends the use of CPAP to improve oxygenation in patients with diffuse pneumonia.

\section{Level of evidence $2+$}

\section{Recommendation}

- Continuous positive airway pressure should be considered for patients with pneumonia and type I respiratory failure who remain hypoxaemic despite optimum medical therapy and oxygen. (Grade C)

\section{Non-invasive ventilation}

In patients with community-acquired pneumonia, NIV has been demonstrated to reduce respiratory rate, tracheal intubation rate and mean duration of Intensive Care Unit (ICU) stay significantly, and in a small subgroup of patients with underlying COPD, to produce an increase in 2-month survival. ${ }^{265}$ Current BTS guidance ${ }^{76}$ recommends the use of NIV as an alternative to tracheal intubation in patients with pneumonia who become hypercapnic.

\section{Level of evidence $2+$}

\section{Recommendation}

- Non-invasive ventilation can be considered for selected patients with community-acquired pneumonia and type II respiratory failure, especially those with underlying COPD. (Grade C)

It should be noted that caution is advised as many patients with acute pneumonia and hypoxaemia resistant to high flow oxygen will require intubation, and that trials of NIV or CPAP should only occur in HDU and ITU settings. ${ }^{76}$

Good practice points

- Physiotherapists involved in the delivery of non-invasive ventilation need to ensure that their practice remains in line with current guidance.

- Personnel involved with the delivery and care of patients using non-invasive ventilation should be adequately trained in the principles, assessment and effects of non-invasive ventilation.

- Patients on continuous positive airway pressure or noninvasive ventilation should be carefully monitored for signs of deterioration and appropriate action taken.

\section{Intermittent positive pressure breathing}

IPPB is a commonly used physiotherapy technique (a form of non-invasive positive pressure ventilation with an integral nebuliser) which has been demonstrated to increase tidal volume, ${ }^{86-89}$ reduce work of breathing if the machine is set up correctly $^{85}$ and improve alveolar ventilation ${ }^{86}$ in patients with COPD. The use of IPPB was investigated in uncomplicated pneumonia ${ }^{266}$ (every $4 \mathrm{~h}$ in the first $24 \mathrm{~h}$, using $1-5$ drops of adrenaline in $5 \mathrm{ml}$ of water in the nebuliser chamber), in combination with chest physiotherapy (postural drainage, percussion, vibration, deep breathing and coughing). There was no significant difference in the resolution rate between treatment groups and controls, who received antibiotics, oxygen therapy and humidification, and were directed by their physicians to deep breathe and cough spontaneously. The treatment arm of this study does not reflect practice in the UK.

\section{Level of evidence 1+}

Recommendation

- Patients admitted with primary uncomplicated pneumonia should not be treated with traditional airway clearance techniques and intermittent positive pressure breathing in combination. (Grade B)

Research recommendation

- Further research is required on the effects of physiotherapy in patients with pneumonia.

\section{Complementary therapy}

Most physiotherapists would not use or consider complementary therapies in patients with the acute conditions described in this section. However, for patients with chronic problems some therapies may be worthy of consideration. For discussion of complementary therapy techniques, please see Web Appendix 1.

\section{SECTION 6 NEUROMUSCULAR DISEASES AND MUSCULOSKELETAL DISORDERS OF THE CHEST WALL SECTION 6a Chest wall disorders}

\section{Introduction}

Chest wall deformity may arise from idiopathic causes in childhood or be acquired due to disease, such as postpoliomyelitis or ankylosing spondylitis, or following thoracoplasty for TB. The most common deformities that give rise to a marked restrictive pattern on spirometry are scoliosis and kyphoscoliosis. Mild scoliosis, as measured by a Cobb angle of $<25^{\circ}$, does not impair respiratory function or limit exercise tolerance. Those with scoliosis in the intermediate range (a Cobb angle of between 25 and $60^{\circ}$ ) have mildly diminished lung volume and a reduction in exercise capacity, which may be due to deconditioning rather than a true ventilatory limitation. ${ }^{267}$ This may be as a result of lack of willingness to participate in aerobic activities, possibly arising from fear of injury or embarrassment associated with body image, primarily a problem of adolescence. ${ }^{267}$

Individuals with severe chest wall deformity, such as kyphoscoliosis and with Cobb angles of $>60-70^{\circ}$, are at an increased risk of developing respiratory failure and pulmonary hypertension, ${ }^{267} 268$ and cor pulmonale when the Cobb angle exceeds $100^{\circ}{ }^{267}$ This is due primarily to the restrictive defect imposed by reductions in both thoracic cage and lung compliance. ${ }^{269}$ Thus any attempt to increase tidal volume $\left(\mathrm{V}_{\mathrm{T}}\right)$ is associated with a greater oxygen cost of breathing. Severe chest wall deformity can also give rise to altered respiratory muscle activity, as the muscles may not be working at an optimal length/tension ratio, reducing their force-generating 
capacity. The resultant reduced inspiratory capacity (IC) results in significant respiratory limitation when exercise is undertaken, which can lead to oxygen desaturation. ${ }^{270}$ Such patients have impaired exercise capacity and may be disabled by breathlessness. One study ${ }^{271}$ reports the benefits of surgical correction and the implications of this for the improvement in respiratory muscle function.

\section{Pulmonary rehabilitation}

The rationale for $\mathrm{PR}$ remains the same in non-COPD as for COPD, although there is scant published evidence for the effectiveness of PR for patients with restrictive chest wall disorders. In the few available studies, however, the results for non-COPD patients are as good ${ }^{254-256}$ or better ${ }^{257} 258$ than for COPD, including for patients with chest wall disorders following post-TB thoracoplasty, ${ }^{255}$ or scoliosis and kyphoscoliosis due to a variety of conditions. ${ }^{258}$ One study compared the effectiveness of a 9-week PR programme in a group of post-TB thoracoplasty patients, with an $\mathrm{FEV}_{1}$-matched group of COPD patients. ${ }^{255}$ Similar significant improvements were seen in both groups of patients for $6 \mathrm{MWD}$, activity scores, dyspnoea and MRC grading. All current guidance recommends including non-COPD patients in $\mathrm{PR}$ and that the content of education sessions should be adjusted accordingly. ${ }^{14} 4647$

\section{Level of evidence $2++$}

Recommendations

- Offer patients with chest wall restriction post-tuberculosis pulmonary rehabilitation. (Grade B)

- Offer patients with chest wall deformity from other causes with reduced exercise capacity and/or breathlessness on exertion pulmonary rehabilitation. (Grade C)

Good practice point

- Pulmonary rehabilitation sessions for patients with chest wall restriction should include relevant education sessions.

\section{Ambulatory oxygen}

In a single study of 12 stable patients with severe to moderate kyphoscoliosis, ${ }^{270}$ significant falls in oxygen saturation during exercise were corrected with the addition of ambulatory oxygen. In addition, breathlessness and recovery time to baseline saturation were significantly improved.

\section{Level of evidence 4}

\section{Recommendation}

- Assess patients with moderate to severe kyphoscoliosis who desaturate on exercise for ambulatory oxygen. (Grade D)

Research recommendation

- Further research into the use of ambulatory oxygen should be undertaken in this client group.

\section{Breathing exercises and respiratory muscle training.}

Two case studies of formal respiratory muscle training were described $^{269}$ with resistance progressed to tolerance. In one subject, this training was used to facilitate weaning from ventilatory assistance. In the other, increase in respiratory muscle strength was gained, with subjective improvement in exercise tolerance.

Postsurgical correction of kyphoscoliosis with the insertion of a Harrington rod, ${ }^{271}$ a greater improvement in VC was gained $(12 \%)$ in the group performing daily deep breathing exercises, both with and without resistance, compared with the control group $(3.4 \%)$ who received no breathing exercises. Inspiratory resistance was created by the application of external resistance, either manually or with a chest strap. Expiratory resistance was created by balloon blowing, in effect a form of PEP. Six-month follow-up demonstrated a slight narrowing of the difference between groups. However, the study included a mixed population of adults and children and is hard to draw firm conclusions from.

\section{Level of evidence 3}

Recommendation

- Consider respiratory muscle training in patients with kyphoscoliosis. (Grade D)

Research recommendations

- Further research into the use of breathing exercises and their effects should be undertaken in this client group.

- Further research into the use of resisted inspiratory and expiratory breathing exercises (including formal respiratory muscle training) should be undertaken in this client group.

- Further research into the use of both resisted and unresisted, inspiratory and expiratory, breathing exercises should be undertaken in this client group when undergoing surgical correction of kyphoscoliosis with a Harrington rod.

\section{Thoracic mobility exercises}

One study ${ }^{268}$ examined the effect of thoracic mobility exercises on VC and chest expansion in a large mixed population cohort ( $n=271$ adults) with idiopathic scoliosis. The exercises undertaken were intensive but were only identified as the Schroth Method and are not described. VC improved in those individuals with a Cobb angle of $>25^{\circ}$, by a mean of $15 \%$ in the group aged $18-24$ and $14 \%$ in those aged $>24$ years; chest expansion increased by $20 \%$ in all groups. There is insufficient evidence to support or refute the use of thoracic mobility exercises in this patient group.

Level of evidence 2 -

Research recommendation

- Further research into the use of thoracic mobility exercises and their effects on vital capacity, total lung capacity and clinical outcome should be undertaken in this patient group.

\section{SECTION 6b Spinal cord injuries}

\section{Introduction}

Upper spinal cord injury results in paralysis of the muscles of the nerve segment at the level of the lesion and below, impacting dramatically on the mechanics of breathing. Complete lesions of the spinal cord affect both inspiratory and expiratory muscles, with the degree of respiratory impairment related to the level of the lesion. Below C3-4, the diaphragm function remains intact, but loss of other respiratory muscles causes abnormalities in all respiratory volumes and flows. ${ }^{272}$ VC decreases to approximately $50 \%{ }^{273}$ and TLC to approximately $70 \%{ }^{273} 274$ of predicted normal values. There is reduction in expiratory reserve volume (ERV) and lung compliance, while RV increases. ${ }^{275} 276$

The abdominal muscles play an important role in normal breathing, as well as in huffing and coughing. ${ }^{277}$ Normal abdominal muscle tone increases intra-abdominal pressure to help elevate the diaphragm for optimal mechanical advantage for inspiration. ${ }^{278}$ Contraction of the abdominal muscles results in increased intra-abdominal pressure and therefore pleural pressure, to help reduce lung volume and assist expiration. Paralysis of these muscles means the abdomen moves paradoxically during expiratory manoeuvres and, combined with paralysis of the other expiratory muscles, this greatly impairs the ability to generate forced expiration, essential for clearing 
bronchial secretions. ${ }^{279}$ Contraction of the clavicular portion of the pectoralis major also plays a part in the active expiration of the upper rib cage and may be intact in patients with lesions between C5 and C8.

The higher the spinal cord lesion, the greater the reduction in both MIP and MEP, measured at the mouth. ${ }^{280}$ A normal cough (a peak cough flow $>360 \mathrm{l} / \mathrm{min}$ with an open glottis) requires inspiration to $85-90 \%$ of TLC, followed by glottal closure to generate sufficient intrathoracic pressures. ${ }^{281}$ When peak cough flow falls below $160 \mathrm{l} / \mathrm{min},{ }^{282}$ secretions accumulating in the airways cannot be effectively cleared. ${ }^{283}$ In complete lesions above T6, the autonomic nervous system is involved, which induces bronchial hypersecretion; this further aggravates the problem. ${ }^{284}$

This restrictive pattern, combined with breathing against an increased load, as a consequence of reduced compliance of the chest wall and increased abdominal compliance, leads to increased work of breathing. Together with respiratory muscle weakness, this contributes to the development of inspiratory muscle fatigue. This has the potential to result in chronic respiratory insufficiency, dependent on the level and completeness of the upper spinal cord injury. ${ }^{285}$ Furthermore, reduced IC, combined with inefficiency of secretion clearance, leads to hypoventilation and sputum retention, causing microatelectasis, or even major segmental or lobar collapse. This can increase susceptibility to infection ${ }^{286}$ and may in turn lead to hypoxia due to ventilation-perfusion mismatching. Further complicating factors arise from mechanical ventilation and enforced immobility. Respiratory complications therefore produce some of the main causes of morbidity and the prime cause of mortality in patients with upper spinal cord injury. Mortality is increased by $30 \%$ in quadriplegics who develop atelectasis or pneumonia. ${ }^{287}$ Clinical practice guidelines ${ }^{288}$ suggest monitoring the following indicators for the development of atelectasis or infection:

- Rising temperature

- Change in respiratory rate

- Shortness of breath

- Increasing pulse rate

- Increasing anxiety

- Increasing volume and tenacity of secretions

- Frequency of suctioning

- Reducing vital capacity

- Declining peak expiratory flow rate, especially during coughing.

\section{Level of evidence $1++$}

Recommendation

- Monitor the patient with spinal cord injury for the signs and symptoms of respiratory problems and take appropriate action if abnormal or changing. (Grade A)

It is worth bearing in mind that the patient may be unaware of deteriorating respiratory status and may not display the typical expected clinical signs. Moreover, sleep-disordered breathing is considered to be a confounding problem, in particular for those with cervical cord injury with a resultant complex sleep apnoea. Bowel dysfunction may also cause further compromise in respiratory function, and the appropriate investigations should be considered.

In the management of upper spinal cord injury, therefore, the principal objective is to minimise preventable respiratory complications, as well as maintaining joint mobility and soft tissue length.
Roth et $a^{289}$ stress the importance of VC monitoring as a single global measure of overall ventilatory status in upper spinal cord injury since it correlates well with all other lung function tests and enables early recognition of deterioration in ventilatory capacity. Suggested cause for concern is when VC falls to 1 litre, potentially signposting the need for ventilatory support.

\section{Level of evidence 4}

\section{Recommendations}

- Measure vital capacity routinely in the patient with upper spinal cord injury and take appropriate action if falling. (Grade D)

- Alert medical staff if vital capacity falls to 1 litre or less. (Grade D)

Good practice point

- Consider the use of an incentive spirometer to monitor vital capacity at home.

\section{Body positioning}

Physiotherapists use positioning to affect ventilation and perfusion, pulmonary volumes, drainage of secretions and the performance and efficiency of respiratory muscles. Paradoxically, in quadriplegia, as a result of loss of abdominal muscle tone and an intact diaphragm, diaphragmatic excursion is augmented in the supine position, thereby increasing VC. ${ }^{290} 291$ However, if a patient is poorly positioned with a resulting increased load on the respiratory muscles, oxygen demand may increase.

Reverse Trendelenberg (see Glossary, Appendix A) and prone positioning are used in practice to improve oxygenation and improve diaphragmatic excursion. Ali and $\mathrm{Oi}^{292}$ found the optimal position for the lungs in upper spinal cord injury patients to be supine or head-up $30^{\circ}$.

\section{Level of evidence 2++}

Recommendations

- Consider the supine position to maximise vital capacity. (Grade B)

- Assess the head-up $30^{\circ}$ position for improving pulmonary function. (Grade C)

Patients with acute quadriplegia may exhibit flaccid paralysis of the upper chest wall, leaving the diaphragm as the only muscle of respiration, creating an intrapleural pressure gradient, preferentially ventilating the lung bases. ${ }^{293}$ Perfusion, however, remains gravity dependent; thus, when an individual with acute quadriplegia is placed in a head-down position (eg, for secretion clearance), acute hypoxaemia may be created as a result of ventilation-perfusion mismatch. The authors ${ }^{293}$ note that it is more significant in patients with injury of less than 1 year duration and that it can be easily treated by supplemental oxygen. They further suggest that this improvement in positional hypoxaemia with time may be due to the development of either spasticity or a degree of recovery in the muscles of the upper chest some months postinjury. This return of some muscle tone can help to restore some ventilation to upper lung regions $^{278}$ and may help to improve cough and secretion clearance.

\section{Level of evidence $2+$}

Recommendations

- The head-down position should only be used where there is a demonstrable need and only with extreme caution. (Grade D)

- Any patient, especially those with early spinal cord injury, should be carefully monitored for signs of hypoxaemia in head-down positions. (Grade D) 
- Take comorbidities, and contraindications and precautions to head-down tilt positions into account. (Grade D)

Good practice points

- Patients with spinal cord injury with resting hypoxaemia should be given supplemental oxygen if placed in the headdown position.

- Patient comfort and preference should be taken into account with any position.

- The effect of an abdominal binder, if used, should be taken into consideration.

\section{Abdominal strapping/abdominal binders}

Abdominal binders are belts or straps positioned between the costal margin and the pelvis to limit abdominal expansion. This reduces pressure dissipation and allows the generation of higher intrathoracic pressures. ${ }^{276}$ They are commonly composed of $45 \%$ polypropylene, $30 \%$ stretch fabric and $25 \%$ cotton, are normally $20 \mathrm{~cm}$ wide and are available in three lengths, fastened and adjusted by adhesive hook and loop tape. This design avoids interference with expansion of the thoracic cavity.

The use of abdominal binders in quadriplegic patients with paralysis of the abdominal muscles has been reported to improve VC, ${ }^{276}{ }^{294} \mathrm{FVC}, \mathrm{MEP}^{275}$ and IC. ${ }^{295}$ Two studies found $\mathrm{FVC}^{275}$ or $\mathrm{VC}^{295}$ to remain greater in supine than in sitting, with or without the binder. Despite this finding, subjects reported a preference for using the binder in the sitting position. ${ }^{275}$ Moreover, improvement in cough effectiveness and increased ease of breathing are reported by patients ${ }^{275}$ when using the binder, with no reports of discomfort or untoward side effects. Although of weak methodology, these studies lend weight to the common practice in spinal injury centres of using abdominal binders.

In a study on the effects of a non-elastic binder on breathing pattern during breathing exercises, ${ }^{296}$ an incidental finding of this study demonstrated that RV, FRC and TLC were reduced, and VC increased with the binder in situ. The authors suggest that the decrease in TLC occurred because the reduction in RV is not counterbalanced by the increase in VC. They therefore suggest that patients should be assessed on a case by case basis to ensure that increases in VC can adequately compensate for the reductions in TLC, FRC and RV when using the non-elastic binder.

\section{Level of evidence 4}

\section{Recommendations}

- Assess the effect of an abdominal binder for upright sitting where improvement in either vital capacity or respiratory muscle function is required. (Grade D)

- Patients using non-elastic binders should be monitored closely. (Grade D)

- When using an abdominal binder, the optimal position for the individual patient should be determined. (Grade D)

Research recommendation

- Further research into the use of abdominal binders should be undertaken.

\section{Cough and airway secretions management}

Coughing and forced expiration with an open glottis (huff) are considered the most important clearance mechanisms for airway secretions. When huff and cough are performed at lower lung volumes, dynamic airway compression creates increased flow velocity through peripheral airways, aiding transport of secretions to more central airways. ${ }^{283}$ Although expiratory muscle function is totally lost in patients with an upper cervical
(C1-4) cord injury, those with transection between C5 and C8 retain some function in the clavicular portion of pectoralis major. ${ }^{276}{ }^{283}$ Contraction of pectoralis major produces compression of the upper rib cage, generating higher intrathoracic pressures thus creating dynamic airways compression in larger airways. This produces higher air flow velocity, increased ERV and reduced RV. ${ }^{283}$ Specific strength training of the clavicular portion of pectoralis major can be undertaken in these patients (see Respiratory muscle training in this section).

Improved efficacy of a spontaneous cough effort may be experienced by adopting a forward lean position when seated, thus raising intra-abdominal pressure and enhancing expiratory flow, with the use of "hooking" an arm over the back of the wheelchair to enable some leverage and fixing of position.

Good practice points

- Try the forward lean position to enhance the effectiveness of spontaneous coughing.

- Try "hooking" one arm over the back of the wheelchair for added stability and leverage during spontaneous coughing.

\section{Manually assisted coughing}

Even when patients are able to clear secretions from larger airways independently, assistance may be necessary to produce an increase in velocity of expiratory flow sufficient to mobilise secretions from smaller airways. Assisted coughing commonly refers to the combined techniques of manual or mechanical maximum insufflation strategies, with manually and/or mechanically assisted coughing. However, evidence for these strategies in this client group was found only for manually assisted coughing and mechanical in-exsufflation. For further information on other techniques, please see Section $6 c$ (neuromuscular disease).

Manually assisted coughing significantly increases peak cough flow ${ }^{297} 298$ by a well timed thrust from an assistant during the expiratory cycle. Hand placement can be on the abdomen (Heimlich-style thrust), anterior chest wall or costophrenic angles (thoracic compression). ${ }^{299}{ }^{300}$ Further description of these techniques can be found in Massery. ${ }^{301}$ In a study of manually assisted coughing in spinal cord injury patients, ${ }^{298}$ both the type of thrust and the body position, supine or upright sitting, were compared. The greatest effects were found in the sitting position and from the Heimlich-style (abdominal thrust) in either position.

\section{Level of evidence 3}

\section{Recommendations}

- Try manually assisted coughing for patients with an ineffective cough. (Grade D)

- The upright seated position should be considered initially. (Grade D)

- The abdominal thrust (Heimlich-style manoeuvre) should be considered initially. (Grade D)

Good practice point

- Alternative body positions and thrusts should be tried if these fail to produce an effective result.

\section{Mechanical insufflation-exsufflation}

Mechanical in-exsufflation provides alternating positive and negative pressure to provide both a larger $V_{\mathrm{T}}$ and assistance with expiration, to improve the effectiveness of mucus clearance. ${ }^{302} 303$ It can be applied via an oral-nasal interface, or an endotracheal or tracheostomy tube. Mechanical inexsufflation is commonly accompanied by manually assisted coughing. 
Pressure cycles of between $+60 \mathrm{~cm} \mathrm{H}_{2} \mathrm{O}$ and $-60 \mathrm{~cm} \mathrm{H}_{2} \mathrm{O}$ are titrated to suit the individual, but should be started low and increased gradually. There are no reports of any serious side effects, although Bach ${ }^{281}$ suggests caution in patients with acute upper spinal cord injury who may be susceptible to bradycardia. Moreover, patients with spinal cord injury may have a low resting blood pressure and syncope. High pressures, therefore, should not be used without very good reason.

\section{Level of evidence 3}

\section{Recommendations}

- Mechanical insufflation-exsufflation should be considered for individuals with upper spinal cord injury, if simpler techniques fail to produce an adequate effect. (Grade D)

- Where cough effectiveness remains inadequate with mechanical insufflation-exsufflation alone, combine it with manually assisted coughing. (Grade D)

Good practice points

- Caution should be observed in patients with acute upper spinal cord injury who may be susceptible to bradycardia or cardiovascular instability.

- Mechanical insufflation-exsufflation pressures should be set to obtain the optimal airway clearance effect in an individual, but avoid using high pressures where possible.

- End the treatment session with an insufflation to minimise airway closure.

\section{Functional electrical stimulation}

Functional electrical stimulation to abdominal muscles significantly improves $\mathrm{FEV}_{1}, \mathrm{FVC}$ and PEF, ${ }^{304} 305$ and has been shown to be significantly better than respiratory muscle training in improving FVC and $\mathrm{FEV}_{1}{ }^{304}$ with greater values produced in supine than in sitting. Linder ${ }^{306}$ used functional electrical stimulation to the abdominal muscles in patients with spinal cord injury and found an increase in the MEP in line with manually assisted coughing, compared with spontaneous cough. An RCT in patients with spinal cord injury studied the effects of 4 weeks of functional electrical stimulation to the clavicular portion of pectoralis major and the abdominal muscles. ${ }^{307}$ Patients in the active therapy group had significant improvements in PEFR, FEV 1 , FVC, MEP and MIP, and suffered fewer pulmonary complications in the follow-up period.

There is no consensus on optimal electrode placement or frequency, although $50 \mathrm{~Hz}$ and 250-300 $\mu$ s were commonly used. ${ }^{304-306} 308$

\section{Level of evidence $2+$}

\section{Recommendation}

- Consider electrical stimulation of the abdominal muscles as a possible means of enhancing lung volumes and cough effectiveness. (Grade C)

Research recommendation

- Further research is required into the clinical effects of functional electrical stimulation and the optimum electrode placements and electrical frequency.

\section{General exercise}

A survey of 308 patients found wheelchair athletes to be significantly less breathless than their non-athletic counterparts, with no identifiable improvement in respiratory muscle performance or pulmonary function, ${ }^{309}$ implying that breathlessness and exercise tolerance are improved in those who actively participate in exercise, although by what mechanism is unclear.

\section{Level of evidence $2+$}

\section{Recommendation}

- Active exercise should be encouraged in patients confined to a wheelchair as a result of spinal cord injury. (Grade D)

\section{Breathing exercises-resisted and unresisted}

Patients who undertook deep breathing exercises, with or without resistance (PEP alone and PEP with inspiratory resistance), significantly increased TLC, VC and RV, but not FRC, compared with a control group. ${ }^{310}$ Interestingly, inspiratory and expiratory flow, alveolar ventilation, respiratory rate and $V_{\mathrm{T}}$ were found to be highest in the deep breathing without resistance group.

\section{Level of evidence $2+$}

Recommendation

- Deep breathing exercises should be encouraged in patients with spinal cord injury. (Grade D)

Research recommendation

- Further research is required into both the nature and comparative effects of deep breathing and resisted breathing exercises in patients with spinal cord injury.

\section{Respiratory muscle training}

A 1999 review of respiratory muscle training ${ }^{311}$ concluded that either inspiratory or expiratory muscle training and EMG biofeedback had a positive effect on respiratory function and respiratory muscle endurance, but the possibility that spontaneous recovery, or a training effect due to the measurement procedures, was responsible for the changes observed cannot be excluded. The authors ${ }^{311}$ noted that respiratory muscle training in two small studies allowed patients to tolerate up to $35 \mathrm{~min}$ off the ventilator, which had the added benefit of improving quality of life and the potential to survive accidental disconnections from ventilation. A 2005 review concluded that historical and current rehabilitation status was not consistently acknowledged and could be considered a confounding factor. ${ }^{312}$

A 2006 systematic review ${ }^{285}$ concludes that, although there tends to be an improvement in expiratory muscle strength and endurance, as well as VC and RV, insufficient data were available to draw firm conclusions about the significance of these improvements in clinical outcomes.

\section{Level of evidence 1+}

Research recommendation

- Further research is required to establish the clinical benefit of inspiratory muscle training for patients with upper spinal cord injury.

Despite the lack of substantive evidence of clinical effect, there are a number of studies identifying that either or both strength and endurance of the respiratory muscles and/or pulmonary function can be improved in patients with upper spinal cord injury with IMT, irrespective of the time postinjury, ranging from only a few weeks to years postinjury. ${ }^{287}$ 303-305 312-320 A positive correlation was observed between protocol adherence and the degree of improvement in MIP. ${ }^{315}$ Furthermore, adherence to training regimens also corresponded positively with the degree of care given by others. ${ }^{315} 321$

A small cohort study ${ }^{316}$ demonstrated an increase in MIP and fewer episodes of sleep-disordered breathing following IMT. Both MIP and MEP significantly increased following resistive IMT, ${ }^{315} 319$ as did diaphragmatic endurance. ${ }^{280}$ Expiratory muscle strength was not altered following IMT. ${ }^{322}$

A single case study ${ }^{321}$ of PEP and IMT combined produced an improvement in respiratory muscle force (both inspiratory and expiratory), $\mathrm{FVC}$ and $\mathrm{FEV}_{1}$. In addition there was a reduction in 
the frequency of respiratory exacerbations, requirement for suctioning and the need for supplementary oxygen. It is not clear, since the techniques were used in parallel, whether either technique or the combination was responsible for the effects.

In a small RCT in patients with upper spinal cord injury, ${ }^{317}$ both the test and the control groups demonstrated an improvement in $\mathrm{V}_{\mathrm{T}}$, MIP and sustained inspiratory pressure from baseline, but differences between groups were not significant. The authors ${ }^{317}$ concluded that it was compensatory breathing strategies and a learning effect for breathing against a resistive load, rather than the IMT itself, that may have had the greater impact on lung volumes and respiratory muscle strength, resulting in a slower deeper respiration during tidal breathing.

Progressive loading and training of the accessory muscles of respiration, using either a respiratory muscle trainer ${ }^{318}$ or an arm-cranked cycle ergometer, ${ }^{313}$ significantly improved VC, $\mathrm{MEP},{ }^{318} \mathrm{FVC}$ and maximal voluntary ventilation, ${ }^{313}$ with a trend for improvement in RV. ${ }^{320}$ Furthermore, respiratory muscle endurance was improved using the arm-cranked ergometer in individuals with thoracic spinal cord injury, to a level similar to the baseline values of a healthy control group. ${ }^{313}$ Moreover, some studies report subjective improvement in feelings of breathlessness and general fatigue. ${ }^{315}{ }^{319}$ However, in one study, despite improvements in PEFR of $11 \%$, from 371 to 412 1/min, following inspiratory and expiratory muscle training using a mask, ${ }^{314}$ there were no significant improvements in FVC, FRC, RV, TLC or FEV 1 .

Abdominal weighting exercises and respiratory muscle training improved pulmonary function, in particular VC, in the early stages of postlesion recovery, evaluated using EMG. ${ }^{287}$ Both methods had a slight impact on either diaphragmatic or accessory muscle strength, respectively, but which did not reach statistical significance, perhaps due to low power $(n=11)$. This study excluded patients with motor return or abdominal muscle activity but acknowledges that spontaneous recovery may have occurred.

\section{Level of evidence $2+$}

Recommendations

- Inspiratory muscle training may be considered for patients with upper spinal cord injury to improve respiratory muscle strength. (Grade C)

- Inspiratory muscle training may be considered for patients with upper spinal cord injury to improve vital capacity and residual volume. (Grade $\mathrm{C}$ )

- Training of the accessory muscles of respiration with progressive loading should be considered. (Grade D)

Research recommendation

- Further research is required in the use of respiratory muscle training in patients with spinal cord injury to establish the optimum type, frequency and duration.

\section{Strength training for the clavicular portion of the pectoralis major}

The clavicular portion of the pectoralis major plays a part in the active expiration of the upper rib cage. ${ }^{320}$ Following a specific training programme for this muscle, a statistically significant improvement in its strength and an increase in ERV was identified, which was preserved for a further 10 weeks following cessation of the training programme. ${ }^{320}$ The authors concluded that this may have been due to the fact that the participants were highly motivated and may have continued with exercising, with strength preserved as a consequence of more regular use of the muscle in activities of daily living. They postulated that these improvements in expiratory muscle strength may improve cough at lower lung volumes and subsequent clearance of peripheral bronchial secretions. ${ }^{320}$ However, there is insufficient evidence to support or refute the use of specific training for the clavicular portion of the pectoralis major muscle.

\section{Level of evidence 2 -}

Research recommendation

- Further research is required in the use of specific training for the clavicular portion of the pectoralis major muscle in patients with spinal cord injury.

\section{SECTION 6c Neuromuscular disease}

\section{Introduction}

Neuromuscular disease covers a wide range of disorders that give rise to progressive muscular weakness. ${ }^{323}$ Common diseases in this category include motor neuron disease (MND), (known as amyotrophic lateral sclerosis in the USA), multiple sclerosis (MS), spinal muscular atrophy (SMA), congenital myopathy, postpoliomyelitis and muscular dystrophies, the most common of these being Duchenne muscular dystrophy (DMD). They vary in age of onset, rates of progression and patterns of muscles involved. ${ }^{324}$

Patients with neuromuscular disease may have a reduced VC due to reduced inspiratory muscle strength, with resultant underventilation of lung units and microatelectasis. ${ }^{318} 325$ The combination of loss of respiratory muscle strength, ineffective cough and decreased ventilation leads to pneumonia, atelectasis, and respiratory insufficiency and failure. ${ }^{324} 326327$ Additionally, the unstretched chest wall muscles shorten and stiffen due to the pathological process of disease and an inability to be worked through the full range. ${ }^{325}$ Such patients will frequently have inadequate peak cough flow due to weakness in inspiratory and expiratory muscles.

Good practice points

- Monitor vital capacity in patients with neuromuscular disease to guide therapeutic interventions.

- When vital capacity falls to $<50 \%$ take appropriate action to minimise the risk of respiratory failure and cough impairment.

\section{Risk with oxygen therapy}

Patients with diaphragmatic dysfunction have been shown to develop severe hypercapnia in response to low-flow (high $\mathrm{FiO}_{2}$ ) oxygen therapy due to increases in ventilation-perfusion mismatching exacerbated by the inability to increase $\mathrm{V}_{\mathrm{T}} \cdot{ }^{328}$ However, this tendency was attenuated with the administration of NIV. ${ }^{32}$ Current BTS guidance warns of the danger in using low-flow oxygen in patients with neuromuscular disease..$^{92}$

\section{Level of evidence $1++$}

\section{Recommendations}

- Low-flow (high $\mathrm{FiO}_{2}$ ) oxygen therapy should be avoided or used with extreme caution due to the risk of carbon dioxide retention in patients with neuromuscular disease. (Grade A)

- Consider non-invasive ventilation as an initial intervention in patients with, or at risk of developing, hypercapnia. (Grade D)

Good practice points

- Monitor oxygen saturation in patients with neuromuscular disease to guide therapeutic interventions.

- Repeat blood gas analysis, or end-tidal $\mathrm{CO}_{2}$ monitoring if available, should be performed 30 min postadministration of newly administered low-flow oxygen therapy in the at-risk patient group. 
- Routinely assess for signs of hypoventilation in this patient group (see Appendix B).

- The delivery of non-invasive ventilation must remain in line with current guidance.

- Personnel involved with the delivery and care of patients using non-invasive ventilation should be adequately trained in the principles, assessment and effects of non-invasive ventilation.

\section{Cough and airway secretion management}

An intact cough mechanism is important for clearing airway debris. ${ }^{329}$ Patients with neuromuscular disease may have an impaired cough and reduced peak cough flow as a result of inspiratory, expiratory and/or bulbar muscle weakness. ${ }^{326}$ 330-339 The consequent reduction in peak cough flow leads to ineffective airway clearance which can accelerate the development of respiratory failure and death. ${ }^{330} 331334338$ For a description of performing peak cough flow, please see Appendix C.

\section{Level of evidence $2+$}

Recommendation

- Peak cough flow should be measured regularly in patients with neuromuscular disease. (Grade D)

Bach and Saporito ${ }^{329}$ conducted a mixed population study in 49 patients which found that those with peak cough flow below $160 \mathrm{l} / \mathrm{min}$, irrespective of the ability to breathe, failed extubation or decannulation. The authors conclude that peak cough flow needs to be $\geqslant 160 \mathrm{l} / \mathrm{min}$ for successful extubation or decannulation of patients with neuromuscular disease. Peak cough flow $>160 \mathrm{l} / \mathrm{min}$, therefore, is believed to be sufficient to clear airway debris successfully, with $<160 \mathrm{l} / \mathrm{min}$ thought to be ineffective. ${ }^{326}{ }^{340}$ It is clear, therefore, that measuring the ability to generate adequate peak cough flow is key to the successful management of this patient group.

Respiratory muscle strength and consequently peak cough flow may reduce during a chest infection as a result of respiratory muscle function deterioration. ${ }^{326}{ }^{341}$ However, it is of particular importance at these periods of airway infiltration that peak cough flow remains adequate to clear airway debris and thus prevent pulmonary complications. ${ }^{340}$ Patients who have a peak cough flow of $>270 \mathrm{l} / \mathrm{min}$ (assisted or unassisted) when well have little risk of developing respiratory failure during a respiratory tract infection. ${ }^{326} 331$ Peak cough flow must be greater therefore than the critical level of $160 \mathrm{l} / \mathrm{min}$ when the patient is clinically stable to avoid the risk of respiratory failure during an infection. Please see Web Appendix 10 for a patient action plan and Appendix B for an algorithm to assist with clinical decision-making.

\section{Level of evidence 4}

\section{Recommendations}

- Measure peak cough flow additionally at the time of an acute respiratory tract infection. (Grade D)

- When peak cough flow is equal to or less than $270 \mathrm{l} / \mathrm{min}$ in a medically stable patient, introduce strategies for assisted airway clearance to raise it above $270 \mathrm{l} / \mathrm{min}$. (Grade D)

- When peak cough flow is equal to or less than $160 \mathrm{l} / \mathrm{min}$, additional strategies to assist secretion clearance must be used. (Grade D)

- If peak cough flow remains equal to or less than $160 \mathrm{l} / \mathrm{min}$ despite additional strategies, contact medical colleagues to discuss ventilation and/or airway management needs. (Grade D)

Good practice point

- The peak cough flow values above are a useful guide only, and physiotherapists should ensure that the patient's cough is sufficient to clear secretions.

\section{Secretion clearance techniques}

Airway clearance techniques are aimed at helping the removal of secretions. Independent airway clearance techniques are ineffective in patients with compromised respiratory muscles. Conventional techniques such as postural drainage are unlikely to be effective in this client group, ${ }^{340}$ and suction is neither well tolerated nor therefore successful. ${ }^{325} 330340342$ Thus patients with compromised respiratory muscles require assisted coughing, non-invasive assistance and aids for airway clearance. Further information on these techniques can be found on the Institution for Rehabilitation Research and Development website; Online Education, Respiratory Care Protocols: http:// $w w w . i r d . c a / e d u c a t i o n /$ presentation.asp? refname $=\mathrm{e} 2 \mathrm{r} 1$

\section{Strategies to assist secretion clearance}

Assisted coughing encompasses the combined techniques of manual or mechanical maximum insufflation strategies, with manually and/or mechanically assisted coughing. Maximal insufflation capacity, manually assisted coughing and mechanical in-exsufflation are techniques to increase peak cough flow via improvement in either inspiratory or expiratory capacity, or both. NIV devices can also be used to augment inspiration. ${ }^{335} 343$ The use of non-invasive respiratory aids has been shown to help secretion clearance without the need for suction. ${ }^{282}$ Additionally respiratory aids have been shown to decrease hospitalisation rates, ${ }^{331}$ prevent, or delay the need for tracheostomy ${ }^{344}$ and prolong survival when used in conjunction with NIV, 334338 if they are administered when oxygen saturation falls below 95\% at times of respiratory insufficiency.

\section{Level of evidence $2+$}

\section{Recommendation}

- When oxygen saturation falls below $95 \%$ the use of noninvasive ventilation and/or strategies to aid airway clearance should be considered. (Grade D)

Good practice point

- Careful assessment is needed to determine if assistance with inspiration, expiration or both, is required.

\section{Maximum insufflation capacity}

Maximum insufflation capacity is the maximum volume of air that can be held with a closed glottis. This may be achieved with a single insufflation using a mechanical in-exsufflator or a positive pressure device, or via air stacking for the patient who can maintain a closed glottis.

Air stacking involves consecutive insufflations, closing the glottis after each one, to create a maximum insufflation; air may be delivered by a resuscitation bag, glossopharyngeal breathing or with NIV. Unaided breath stacking can be performed by some patients without assistance by increasing the volume of consecutive breaths to a maximum insufflation. Patient representatives, without bulbar muscle involvement, report improvements in their perception of cough strength and secretion management when using unaided breath stacking.

Patients who have poor bulbar function are unable to increase maximum insufflation with air stacking techniques due to an inability to close the glottis. ${ }^{325}{ }^{332}$ Maximum insufflation can therefore only be achieved in one application in patients with poor bulbar function. ${ }^{325} 332$

Performing maximal insufflation prior to coughing, or manually or mechanically assisted coughing, will increase inspiratory volume and consequently the expiratory flow and cough efficiency, providing $44 \%$ of the variance in cough performance from baseline. ${ }^{343}$ The use of maximum insufflation 
techniques prior to manually assisted cough should be undertaken routinely when VC falls below $1500 \mathrm{ml}$, as VC levels below this level lead to ineffective cough generation. ${ }^{325} 330$

The difference between maximum insufflation capacity and VC has been shown to correlate positively with peak cough flow. ${ }^{325}$ Subjects with a larger maximum insufflation capacity/ VC difference consequently have a greater ability to eliminate airway secretions, thus decreasing the risk of pulmonary complications. ${ }^{332}$

Maximal insufflation to IC also provides a full range of movement to the lungs and chest wall. Maximal insufflation to IC performed 15 times three times daily increased maximum insufflation and subsequent peak cough flow when commenced in patients with neuromuscular disease and reduced VC $(<50 \%$ predicted or $<2000 \mathrm{ml}) .{ }^{332}$ Moreover, the improvement seen was despite disease progression, evident by decreasing VC and unassisted peak cough flows throughout the study period. ${ }^{332}$

\section{Level of evidence $2+$}

\section{Recommendations}

- Use some form of maximal insufflation strategy to improve effective cough generation when vital capacity falls below $1500 \mathrm{ml}$ or $50 \%$ predicted. (Grade D)

- Use single maximal insufflation techniques for patients with bulbar dysfunction who are unable to breath stack. (Grade D)

- Teach patients without bulbar muscle involvement unaided breath stacking to improve cough effectiveness independently where possible. (Grade D)

- Regular breath stacking (10-15 times three times per day) to maximal insufflation capacity should be performed by patients with vital capacity of less than $2000 \mathrm{ml}$ or $50 \%$ predicted. (Grade D)

Research recommendation

- Further research is required to establish the relative efficacy of different maximal insufflation strategies in patients both with and without bulbar muscle involvement to improve cough effectiveness.

\section{Glossopharyngeal breathing}

Glossopharyngeal breathing, also known as "gulping" or "frog breathing", involves a series of gulps using the lips, tongue, pharynx and larynx to push air into the lungs when normal inspiratory muscles are not functioning. Glossopharyngeal breathing consists of cycles of 6-10 gulps of air followed by exhalation. There are five distinct steps to one cycle of glossopharyngeal breathing ${ }^{345}$ :

- A mouthful of air is taken, depressing the tongue, jaw and larynx to get maximum volume.

- The lips are closed and the soft palate raised to trap the air.

- The jaw, floor of the mouth and larynx are raised. This, together with progressive motion of the tongue, forces air through the opened larynx.

- After as much air as possible has been forced through the larynx it is closed and the air is retained in the lungs until the next cycle is initiated.

- Exhalation occurs when the glottis opens and the inflated lungs deflate passively due to elastic recoil.

Conditions for which glossopharyngeal breathing has been reported to be helpful are postpoliomyelitis, ${ }^{345-347}$ spinal cord injuries ${ }^{302} 347$ 348-353 and some neuromuscular diseases. ${ }^{354} 355$ Glossopharyngeal breathing can be utilised to augment maximal inspiration in patients who cannot generate adequate inspiratory effort. ${ }^{354}$ It has been reported to increase VC 345348349356357 by anything from $21 \%$ to $39 \%$. $^{346} 352358$ Improving VC can aid in maintaining chest wall range of movement and increased pulmonary compliance. ${ }^{359}$ It has also been shown to increase FVC $^{353} 350$ and peak cough flow, ${ }^{345}{ }^{357}$ which resulted in patients being able to produce a functional cough to enable clearance of secretions from the airways. ${ }^{360}$ Patients have also reported improved voice quality. ${ }^{347} 352$

Ventilator-dependent patients have achieved ventilator-free breathing time after learning the technique, enabling them to develop greater functional independence for short periods. ${ }^{348} 353355356359$ Two papers ${ }^{302} 348$ report that glossopharyngeal breathing can be effectively used in tracheostomy patients to allow for ventilator-free breathing time. It is harder to learn the technique with a tracheostomy in situ, however, due to its effect on laryngeal elevation and epiglottis closure. ${ }^{302} 348350$ Limitations of the technique have also been reported in patients with oropharyngeal weakness.

Most of these papers are small uncontrolled studies or reports, and many are very old as this technique lost popularity with the advent of advanced non-invasive ventilatory support. However, all these papers report positive findings which, combined, make the technique worthy of further study and due consideration in the clinical field. It has also been noted that, although potentially extremely useful, glossopharyngeal breathing is rarely taught because there are few healthcare professionals familiar with it. ${ }^{359}$ Glossopharyngeal breathing is considered difficult and time-consuming to learn and teach. Patient representatives, however, report finding it an extremely useful technique to increase voice strength, maintain independent ventilation for periods of time and help with generating maximum insufflation capacity prior to cough. A DVD/video on the technique (Glossopharyngeal ("Frog") Breathing-what, when and how? produced by Barbara Webber FCSP and Jane Higgens MCSP) is available from Barbara Webber: telephone +44(0)1494 725724 or email bwebber@gotadsl.co.uk.

\section{Level of evidence 3}

\section{Recommendations}

- Consider teaching glossopharyngeal breathing to patients with reduced vital capacity to maintain range of chest wall movement and pulmonary compliance. (Grade D)

- Consider teaching glossopharyngeal breathing as one of the means of achieving maximal insufflation capacity in patients who have difficulty in clearing secretions. (Grade D)

- Consider teaching glossopharyngeal breathing to ventilatordependent patients to allow some ventilator-free breathing time. (Grade D)

- Consider teaching glossopharyngeal breathing to patients with decreased voice strength. (Grade D)

Good practice points

- Physiotherapists involved in the long-term care of patients with neuromuscular disease should consider learning the technique of glossopharyngeal breathing.

- Physiotherapists should include glossopharyngeal breathing more widely in their rehabilitation plan to ensure a more holistic and active programme for the neuromuscular patient.

Research recommendation

- Further study of the effects of glossopharyngeal breathing is required.

\section{Manually assisted coughing}

This is the provision of inspiratory assistance, followed by the augmentation of expiratory effort ${ }^{326}$ (for details, see Section $6 b$ 
Spinal cord injury). Inspiration can be increased using the methods described above in the section on "Maximum insufflation capacity".

In patients without major bulbar impairment, maximal insufflation with manually assisted cough (abdominal thrusts) significantly increased peak cough flow, ${ }^{325}$ with $75 \%$ of the subjects obtaining a peak cough flow $>160 \mathrm{l} /$ min. Trebbia et $a^{343}$ compared maximal insufflation alone with manually assisted cough alone and with the combination of the two. All three techniques improved both VC and peak cough flow significantly, but the greatest improvement was with the combination, being significantly greater than either technique alone. Others have studied manually assisted coughing in comparison with mechanical techniques, and these papers will be discussed in the relevant sections below.

Patient representatives and their carers involved in these guidelines have reported a preference for performing abdominal thrusts with the carer standing in front of the patient. This enables improved communication to synchronise the cough and a better ability to judge from facial expression whether any discomfort is caused. They also report that the combination of breath stacking with manually assisted coughing provides further increases of cough strength and ability to clear secretions. This supports current practice and the preceding guidance.

\section{Level of evidence $2+$}

Recommendation

- Manually assisted coughing should be used to increase peak cough flow in patients with neuromuscular disease. (Grade C)

- Combine manually assisted coughing with a maximal insufflation capacity strategy. (Grade D)

- Abdominal thrusts should be performed standing in front of the patient where possible to assist communication. (Grade D)

\section{Mechanical insufflation-exsufflation}

Mechanical in-exsufflation combines increasing inspiration with facilitation of expiration by alternating positive and negative pressure. ${ }^{339}$ For a fuller description, please see the section with this title under Spinal cord injury. A systematic review of mechanical in-exsufflation ${ }^{342}$ includes three controlled trials comparing mechanical in-exsufflation with other assisted cough techniques in mixed stable patient populations with neuromuscular disease. ${ }^{333} 335358$ Two of these studies ${ }^{335}{ }^{358}$ found that the mean peak cough flow of included subjects increased to levels greater than the $270 \mathrm{l} / \mathrm{min}$ stable threshold, which was not the case for the third..$^{333}$

Although in these studies mechanical in-exsufflation increased peak cough flow to the highest values obtained, it is noteworthy that the use of manually assisted coughing applied postinflation to maximum insufflation capacity (achieved via glossopharyngeal breathing, volume ventilation ${ }^{358}$ or in isolation ${ }^{333}{ }^{335}$ ) increased peak cough flow to above the critical $160 \mathrm{l} / \mathrm{min}$ level needed to clear airway debris. It should be noted that the studies all used different in-exsufflation regimens. Furthermore, only one study ${ }^{358}$ contained any acutely unwell patients $(n=5)$, so it is difficult to ascertain if the settings used in these studies would be effective during an acute chest infection.

Sivasothy et al ${ }^{333}$ did not include exsufflation within the inexsufflation cycle prior to coughing. Significant increases in peak cough flow, however, ${ }^{333}$ were observed with manually assisted coughing alone, mechanical insufflation alone and mechanical insufflation combined with manually assisted coughing, but only in patients who did not have scoliosis. This was not observed in patients with scoliosis, which suggests that the combinations used have little role in increasing peak cough flow in scoliotic patients with neuromuscular disease. However, the numbers in this study were very small so the subanalysis on the presence or absence of scoliosis should be viewed with caution.

One study ${ }^{335}$ used insufflation and exsufflation pressures set to patient comfort, while two others ${ }^{339}{ }^{358}$ set pressure at the maximal tolerated levels. The study in stable MND patients ${ }^{339}$ demonstrated significant increases in peak cough flow from baseline unassisted coughs, in patients both with and without bulbar muscle weakness, when using either manually assisted coughing, exsufflation alone or mechanical in-exsufflation. In those without bulbar muscle involvement, the weakest patients (VC $<50 \%$ ) demonstrated the largest increases in peak cough flow. Only exsufflation performed in isolation increased cough peak flow to values $>270 \mathrm{l} / \mathrm{min}$, but all other interventions achieved cough peak flows of greater than the suggested critical $160 \mathrm{l} / \mathrm{min}$ level. This trend was not seen in the subgroup with bulbar involvement, suggesting that glottic closure is important in achieving cough peak flow. However, although peak cough flow was significantly improved in this subgroup, the mean value did not exceed the $270 \mathrm{l} / \mathrm{min}$ threshold for stable patients, although values obtained were greater than the $160 \mathrm{l} / \mathrm{min}$ critical level for clearing airway debris.

Winck et $a^{337}$ studied different pressures of mechanical inexsufflation in a clinically stable mixed population of neuromuscular disease patients. Subanalysis of the data demonstrated significant increases in peak cough flow with pressures set at $40 \mathrm{~cm} \mathrm{H} \mathrm{H}_{2} \mathrm{O}$ for insufflation and $-40 \mathrm{~cm} \mathrm{H}_{2} \mathrm{O}$ for exsufflation. Again, although peak cough flow was significantly improved, the mean value did not exceed the $270 \mathrm{l} / \mathrm{min}$ threshold for stable patients. However, Chatwin et al ${ }^{335}$ achieved mean peak cough flow values of $2971 / \mathrm{min}$ with much lower pressures (insufflation $15 \pm 3$ and exsufflation $15 \pm 9$ ).

Due to the differences in regimens used and the conflicting results from these studies on the relative effectiveness of the inexsufflator compared with other combinations of techniques, interpretation for comparison of effectiveness of techniques is difficult. Furthermore, the use of clinically stable patients makes it impossible to extrapolate the findings to those with acute respiratory infection.

\section{Level of evidence 4}

\section{Recommendations}

- Consider mechanical insufflation-exsufflation as a treatment option in patients with bulbar muscle involvement who are unable to breath stack. (Grade D)

- Consider mechanical insufflation-exsufflation for any patient who remains unable to increase peak cough flow to effective levels with other strategies. (Grade D)

- Where cough effectiveness remains inadequate with mechanical insufflation-exsufflation alone, combine it with manually assisted coughing. (Grade D)

Good practice points

- Mechanical insufflation-exsufflation pressures should be titrated to suit the individual to optimise the insufflation and exsufflation required to achieve an effective cough.

- If secretions require loosening to facilitate removal, other strategies must be employed prior to using mechanical insufflation-exsufflation. 


\section{Research recommendations}

- Further research is required to establish the effect of mechanical insufflation-exsufflation in patients with neuromuscular disease and acute respiratory infection.

- Further research is required to establish the relative effectiveness of the mechanical insufflation-exsufflator compared with other combinations of techniques.

\section{Intrapulmonary percussive ventilation}

Evidence in paediatric patients with neuromuscular disease and in adult patients postoperatively or with CF F $^{167} 186$ suggests intrapulmonary percussive ventilation to be safe and effective in mobilising secretions, but there is scant evidence in the patient with neuromuscular disease. Birnkrant et al ${ }^{361}$ report in their case series ( 1 adult and 3 paediatric patients with DMD) that one of their four subjects experienced a brief episode of third-degree atrioventricular block, with hypoxaemia and bradycardia, during two intrapulmonary percussive ventilation treatments. However, they concluded that intrapulmonary percussive ventilation is effective in preventing pulmonary consolidation in neuromuscular patients who are not responding to treatment using more conventional therapeutic techniques.

In eight adult (19-23 years) DMD patients ventilated via a tracheotomy, Toussaint et al ${ }^{362}$ compared two treatments for 5 days each, thrice daily, in a randomised crossover trial. Treatment was: the FET, manually assisted coughing, a $0.9 \%$ sodium chloride nebuliser and suction, with and without intrapulmonary percussive ventilation. Intrapulmonary percussive ventilation significantly enhanced the weight of sputum cleared in $69 \%$ of subjects, without any adverse cardiovascular effects. Subjects' initial unassisted peak cough flow was $<150 \mathrm{l} / \mathrm{min}$ and there were no statistically significant differences in improvements in peak cough flow between the two treatments. They concluded that intrapulmonary percussive ventilation increases the effectiveness of assisted mucus clearance techniques. This concurs with other expert opinion. The use of intrapulmonary percussive ventilation is advocated to mobilise secretions, where there is consolidation and collapse on chest $x$ ray, rather than to increase peak cough flow.

The ATS consensus statement on the management of patients with $\mathrm{DMD}^{326}$ concludes there is insufficient evidence to make any firm recommendations on the use of intrapulmonary percussive ventilation with self-ventilating patients, but that the use of airway clearance devices dependent on a normal cough is likely to be ineffective without the concurrent use of other assisted cough techniques. Therefore, other techniques, alone or in combination, may be required to clear secretions once mobilised centrally following intrapulmonary percussive ventilation.

\section{Level of evidence 4}

Recommendations

- Intrapulmonary percussive ventilation may be considered for patients with neuromuscular disease to aid loosening of secretion prior to removal where there is evidence of sputum retention and other techniques have failed. (Grade D)

- In patients with ineffective cough, assisted cough strategies must be used additionally to increase cough effectiveness. (Grade D)

- Patients using intrapulmonary percussive ventilation must be monitored closely during and after treatment for any adverse response. (Grade D)
Research recommendation

- Further research is required to evaluate the safety and efficacy of intrapulmonary percussive ventilation in the care of patients with neuromuscular disease.

\section{Respiratory muscle training}

Respiratory muscle training in neuromuscular disease is based on the assumption that increased strength and endurance of the respiratory muscles will lead to improved and better preserved lung function. ${ }^{326}$ Synthesising findings from the studies included is difficult due to their heterogeneity. Some have included adults only, ${ }^{363-366}$ others both adults and paediatric subjects, ${ }^{367-372}$ and some have included populations with different diseases or severities. Additionally the studies used different respiratory muscle training protocols-for example, duration of respiratory muscle training varied widely, from just $3-5$ weeks $^{364}$ to 2 years. ${ }^{372}$ Frequency ranged from once daily five times a week ${ }^{364}$ to four times daily. ${ }^{363}$ Outcome measures also varied between studies. There is insufficient evidence therefore to support or refute the use of respiratory muscle training or any given training regimen over another in this patient group.

\section{Strength training}

The intensity of respiratory muscle training and its impact on strength varied across the studies reviewed. Subjects with more severe disease - that is, those with $\mathrm{VC}<25 \%$ predicted, elevated carbon dioxide $\left(\mathrm{CO}_{2}\right)$ levels or needing $\mathrm{NIV}^{366}{ }^{370}$ - did not increase respiratory muscle strength with respiratory muscle training, whereas subjects with a VC $>25 \%$ predicted did. ${ }^{370}{ }^{372}$ Benefits were preserved up to 6 months postcessation of the intervention. ${ }^{370}$

Winkler et $a l^{371}$ made an attempt to control for the impact of disease progression in $\mathrm{DMD}$ and SMA subjects by stratifying groups according to decline in VC in the preceding 12 months. They identified a correlation between the number of successfully completed exercises with improvements in both strength and endurance in the group with a $<10 \%$ decline in VC, indicating a dose response in this group. This group contained all three of the SMA patients. No such correlation was found in the group with a $>10 \%$ decline. It should also be noted that subjects in this study had no evidence of hypoventilation or respiratory failure. The findings from these studies suggest that more severely affected patients are already working at their maximal capacity.

\section{Level of evidence 2 -}

\section{Endurance training}

Despite heterogeneity of training and assessment methods across included studies, all demonstrated an improvement in endurance with training. The only long-term study ${ }^{372}$ demonstrated increases in respiratory muscle strength and endurance up to 10 months after commencement of respiratory muscle training in DMD and SMA subjects, at which point the effects stabilised, although the increases in strength and VC were maintained for the whole 3-year training period. This suggests a reduction in the expected VC decline in this patient group and could therefore potentially help delay the commencement of respiratory support.

In one small uncontrolled study of patients with $\mathrm{MS}^{364}$ respiratory muscle training produced increases in both strength and endurance. In another small study, in patients with postpoliomyelitis, ${ }^{366}$ a subjective improvement in the capacity to perform activities of daily living and increased perception of 
well-being were reported post-training. It must be borne in mind, however, that some of the improvements seen in these studies could be due to the learning effect of the tests of respiratory muscle strength.

\section{Level of evidence 2 -}

Although the methodological quality of the above papers is poor, the papers consistently show trends for improved respiratory muscle strength and endurance postrespiratory muscle training. However, recent physiological evidence suggests that the protective mechanism of nitric oxide release in exercising muscle may be defective in DMD and SMA patients. ${ }^{373}{ }^{374}$ The potential for increased muscle damage during the application of respiratory muscle training cannot be excluded, therefore, in these patient groups. Current recommendations in DMD do not fully endorse respiratory muscle training in this client group and suggest further study. ${ }^{326}$

\section{Level of evidence 4}

\section{Research recommendations}

- Further research is required to determine whether respiratory muscle training is safe and beneficial in patients with Duchenne muscular dystrophy and spinal muscle atrophy.

- Further research is required to determine whether respiratory muscle training is beneficial in patients with other types of neuromuscular disease.

\section{Complementary therapy}

Most complementary therapies used or recommended by physiotherapists are unlikely to be considered for patients with neuromuscular disease. However, in some individuals and for those with chest wall disease, some techniques could be worthy of consideration. For discussion of complementary therapy, please see Web Appendix 1.

\section{SECTION 7 PHYSIOTHERAPY WORKFORCE CONSIDERATIONS}

Workforce planning is an essential part of service delivery, although physiotherapy services in the UK are usually independent of medical directorates. Whilst individual therapists may, for the most part, be permanently or temporarily attached to a particular speciality, not uncommonly the respiratory physiotherapy team manage all patients with respiratory problems regardless of medical speciality or setting. Individual members may therefore "rotate" to another area or be deployed to cross-cover during annual or maternity leave, or sickness absence, to maintain some sort of service across the board in these situations. The degree to which medical units or wards have a named therapist on a permanent basis varies from Trust to Trust. Not uncommonly, except in the larger hospitals, the number of respiratory physiotherapists is small, and loss of an individual physiotherapist to a clinical area has dramatic consequences to the service provision. Moreover, being in such small numbers, specialist respiratory physiotherapists cannot provide 7-day or 24-hour cover. For this reason, physiotherapists from other specialities, in particular junior staff, are used to cover "out-of-hours" care. Every effort is made to train these individuals to an acceptable degree, but it remains a continuing challenge to provide a competent on-call workforce.

This historical and rather complex system has the advantages of (1) no area of the service, unless there are extraordinary circumstances or the setting is very small, is without any sort of service in the absence of the usual team members, and (2) training of new and junior team members, as well as succession planning, is inherent in the system, allowing those who become "respiratory physiotherapists" the chance to learn to handle a variety of respiratory problems, be they in
Table 2 Time (hours) required for physiotherapy interventions in uncomplicated and complex situations

\begin{tabular}{|c|c|c|}
\hline \multirow[b]{2}{*}{ Intervention } & \multicolumn{2}{|l|}{ Time (h) } \\
\hline & $\begin{array}{l}\text { Uncomplicated } \\
\text { patient }\end{array}$ & $\begin{array}{l}\text { Acutely unwell or } \\
\text { complex patient }\end{array}$ \\
\hline \multicolumn{3}{|l|}{$\begin{array}{l}\text { Initial assessment of all patients includes: } \\
>\text { History } \\
>\text { Physical examination }\end{array}$} \\
\hline $\begin{array}{l}\text { Investigations and results } \\
\text { Interpretation of imaging } \\
\text { Tests of mobility, function or exercise } \\
\text { capacity }\end{array}$ & $0.5-0.75$ & 1 \\
\hline \multicolumn{3}{|l|}{ Airway clearance techniques } \\
\hline $\begin{array}{l}\text { Initial exploration and teaching of appropriate } \\
\text { technique }\end{array}$ & $0.5-0.75$ & 1 \\
\hline \multicolumn{3}{|l|}{ Airway clearance techniques } \\
\hline Follow-up/review & $0.25-0.5$ & $0.5-1$ \\
\hline Breathlessness management & 0.50 & 0.75 \\
\hline Self-management/education & 0.25 & 0.50 \\
\hline $\begin{array}{l}\text { Individual help with mobility, physical activity/ } \\
\text { exercise }\end{array}$ & 0.50 & 1 \\
\hline Formal exercise test \pm mobility aids & $0.25-0.50$ & $0.75-1$ \\
\hline Ambulatory oxygen assessment & 1 & $1.25-1.45$ \\
\hline \multicolumn{3}{|l|}{ Non-invasive ventilation } \\
\hline CPAP, NIV, IPPB: set up & 1 & 1 \\
\hline \multicolumn{3}{|l|}{ Non-invasive ventilation } \\
\hline CPAP, NIV, IPPB ongoing & 0.50 & 1 \\
\hline \multicolumn{3}{|l|}{$\begin{array}{l}\text { Special interventions, eg: } \\
\text { Bronchoconstriction trials }\end{array}$} \\
\hline $\begin{array}{l}- \text { Induced sputum } \\
- \text { Hypertonic } \mathrm{NaCl} \text { trial }\end{array}$ & 1 & 1 \\
\hline Pulmonary rehabilitation assessment & \multicolumn{2}{|c|}{1 per patient or 10 per group } \\
\hline Pulmonary rehabilitation & \multicolumn{2}{|c|}{ 10/patient/programme, or } \\
\hline 8-week programme & \multicolumn{2}{|c|}{$100 /$ group or programme } \\
\hline
\end{tabular}

CPAP, continuous positive airway pressure; IPPB, intermittent positive pressure breathing; NIV, non-invasive ventilation.

the Intensive Care Unit, High Dependency Unit, the medical or surgical wards, or in the outpatient setting or patient's home. The system, however, does create difficulties in the identification of numbers of whole time equivalent (WTE) physiotherapists required to provide a service to any given speciality, for example respiratory medicine, vital in today's commissioning climate.

The Cystic Fibrosis Trust (UK) has documented guidelines for the staffing required to achieve adequate care of the CF patient group. ${ }^{375}$ The staffing requirements were developed using working groups and committees of experts in the field to obtain expert opinion. These guidelines recommend two WTE physiotherapists per 50 patients within a specialist unit and 1-2 WTE physiotherapists within a local unit with fewer than 50 patients. It is acknowledged that these requirements may be altered where there are a large number of more unwell or complex patients being cared for or where there is an exceptionally large unit of $>200$ patients. Guidelines on expertise of staff are that they should spend at least half of their working time within care of CF patients in order to maintain expertise.

Given the complexity of the task, it is beyond the scope of this document to provide comprehensive recommendations for WTE physiotherapists for a population of other respiratory patients at this stage. However, because of the urgent need to address this appropriately, a larger exercise on workforce planning needs to take place with the help of the CSP and the BTS. This is already underway and will follow as soon as possible. In the meantime, however, a consensus 
was reached among the steering group, chairs of the guidelines working party and some external experts in managing physiotherapy services, on the clinical component on each physiotherapy intervention and the time needed to undertake the treatment listed in both uncomplicated and complex situations (table 2)

\section{Conclusion}

This is the first extensive systematic literature review undertaken of the existing evidence surrounding comprehensive physiotherapy management of the spontaneously breathing, medical, respiratory, adult patient. Inevitably, given the youth of the academic component of the profession, and lack of funding and infrastructure for research in physiotherapy, much of the evidence is not at the highest level. This is not unique to physiotherapy. These guidelines have highlighted where there is substantial evidence and where there is paucity of evidence. It provides direction for future physiotherapy research. There is, however, considerable evidence supporting the need for physiotherapy across all these diagnostic groups, and this document provides the first step in defining the breadth of respiratory physiotherapy. It demonstrates that the profession encompasses more than "tipping and bashing" and that the integrated approach of physiotherapy embraces a wide variety of techniques, including: breathing re-education, dyspnoea management, physical training and pulmonary rehabilitation, airway clearance, non-invasive ventilation and acupuncture. The physiotherapist should be an integral part of any respiratory team, providing effective and practical management for the benefit of the respiratory patient. These guidelines have also started the process of formulating recommendations for the physiotherapy workforce required to provide the interventions recommended.

Acknowledgements: ACPRC would like to offer grateful thanks to Sheila Edwards for her farsighted approach and, along with her team, for their staunch support of the profession and the project. We are indebted to Dr David Boldy for initiating the creation of these guidelines during his chairmanship of the Standards of Care Committee, and to Dr Norman Johnson and the committee for supporting the work to its conclusion. We are very grateful to Judith Scammell for her painstaking work adding the references and assisting with the document revisions. We would like to thank Sue Pieri-Davies and Drs Michelle Chatwin, Michel Toussaint and John Bach for their constructive help with Section 6, and all those people with lung conditions who offered constructive comments on the content of the guideline and the readability of the patient information leaflets. Finally, our enormous gratitude to Dr Martin Allen, for his unstinting support and efforts to integrate physiotherapy into the multidisciplinary team and the BTS over the last 15 years, and without whom this document would not exist.

\section{REFERENCES}

1. Chartered Society of Physiotherapy. Charting the future of physiotherapy London: CSP, 2008.

2. Chartered Society of Physiotherapy. http://www.csp.org.uk/ (accessed October 2008).

3. Ewart $\mathbf{W}$. The treatment of bronchiectasis and of chronic bronchial affections by posture and respiratory exercises. Lancet 1901;2:70-2.

4. MacMahon C. Breathing and physical exercises for use in cases of wounds in the pleura, lung and diaphragm. Lancet 1915;2:769-70.

5. Scottish Intercollegiate Network (SIGN). Key to evidence statements and grades of recommendations. http://www.sign.ac.uk/guidelines/fulltext/50/annexb. html (accessed October 2008).

6. British Thoracic Society. Guidelines for the management of chronic obstructive pulmonary disease. The COPD Guidelines Group of the Standards of Care Committee of the BTS. Thorax 1997;52:S1-28.

7. Halpin D. NICE guidance for COPD. Thorax 2004;59:181-2.

8. American Thoracic Society. Standards for the diagnosis and care of patients with chronic obstructive pulmonary disease (COPD) and asthma. Am Rev Respir Dis 1987; 136:225-44.

9. Celli BR, MacNee W. Standards for the diagnosis and treatment of patients with COPD: a summary of the ATS/ERS position paper. Eur Respir J 2004;23:932-46.
10. Bekkering GE, Hendriks HJM, Paterson WJ, et al. Guidelines for physiotherapeutic management in chronic obstructive pulmonary disease (COPD). Phys Ther Rev 2000:5:59-74.

11. Gaskell DV, Webber BA. The Brompton Hospital guide to chest physiotherapy. 3rd edn. Oxford: Blackwell Scientific Publications, 1977:78-83

12. Innocenti D. Chest conditions. Physiotherapy 1969;55:181-9.

13. Lum LC. Physiological consideration in the treatment of hyperventilation syndromes J Drug Res 1983;8:1867-72.

14. Nici L, Donner C, Wouters E, et al. American Thoracic Society/European Respiratory Society statement on pulmonary rehabilitation. Am J Respir Crit Care Med 2006;173:1390-1413.

15. Ellis ER, Bye PT, Bruderer JW, et al. Treatment of respiratory failure during sleep in patients with neuromuscular disease. Positive-pressure ventilation through a nose mask. Am Rev Respir Dis 1987;135:148-152.

16. Ellis ER, Grunstein RR, Chan $\mathrm{S}$, et al. Noninvasive ventilatory support during sleep improves respiratory failure in kyphoscoliosis. Chest 1988;94:811-815.

17. Bott J, Baudouin SV, Moxham J. Nasal intermittent positive pressure ventilation in the treatment of respiratory failure in obstructive sleep apnoea. Thorax 1991;46:457-458

18. Bott J, Carroll MP, Conway JH, et al. Randomised controlled trial of nasal ventilation in acute ventilatory failure due to chronic obstructive airways disease. Lance 1993;341:1555-1557.

19. Piper AJ, Parker S, Torzillo PJ, et al. Nocturnal nasal IPPV stabilizes patients with cystic fibrosis and hypercapnic respiratory failure. Chest 1992;102:846-50.

20. Piper AJ, Wilson G. Nocturnal nasal ventilatory support in the management of daytime hypercapnic respiratory failure. Aust J Physiother 1996;42:17-29.

21. Hess DR. Secretion clearance techniques: absence of proof or proof of absence? Respir Care 2002:47:757-8.

22. Bourbeau J, Collet JP, Schwartzman K, et al. Economic benefits of selfmanagement education in COPD. Chest 2006;130:1704-11.

23. Rochester DF, Braun NMT, Laine S. Diaphragmatic energy expenditure in chronic respiratory failure the effect of assisted ventilation with body respirators. Am J Med 1977:63:223-32.

24. Landers MR, McWhorter JW, Filibeck D, et al. Does sitting posture in chronic obstructive pulmonary disease really matter? An analysis of 2 sitting postures and their effect on pulmonary function. J Cardiopulm Rehabil 2006;26:405-9.

25. LaPier TK, Donovan C. Sitting and standing position affect pulmonary function in patients with COPD: a preliminary study. Cardiopulm Phys Ther J 1999:1-7.

26. Barach AL. Chronic obstructive lung disease: postural relief of dyspnea. Arch Phys Med Rehabil 1974:55:494-504.

27. Sharp JT, Druz WS, Moisan T, et al. Postural relief of dyspnoea in severe chronic obstructive pulmonary disease. Am Rev Respir Dis 1980;122:201-11.

28. Druz WS, Sharp JT, Druz WS, et al. Electrical and mechanical activity of the diaphragm accompanying body position in severe chronic obstructive pulmonary disease. Am Rev Respir Dis 1982;125:275-80.

29. O'Neill S, McCarthy DS. Postural relief of dyspnoea in severe chronic airflow limitation: relationship to respiratory muscle strength. Thorax 1983;38:595-600.

30. Heijdra YF, Dekhuijzen PN, van Herwaarden CL, et al. Effects of body position, hyperinflation, and blood gas tensions on maximal respiratory pressures in patients with chronic obstructive pulmonary disease. Thorax 1994:49:453-8.

31. Probst VS, Troosters T, Coosemans I, et al. Mechanisms of improvement in exercise capacity using a rollator in patients with COPD. Chest 2004;126:1102-7.

32. Yohannes AM, Connolly MJ. Early mobilization with walking aids following hospital admission with acute exacerbation of chronic obstructive pulmonary disease. Clin Rehabil 2003;17:465-71.

33. Velloso M, Jardim JR. Study of energy expenditure during activities of daily living using and not using body position recommended by energy conservation techniques in patients with COPD. Chest 2006;130:126-32.

34. Dechman G, Wilson CR. Evidence underlying breathing retraining in people with stable chronic obstructive pulmonary disease. Phys Ther 2004;84:1189-97.

35. Gosselink R. Breathing techniques in patients with chronic obstructive pulmonary disease (COPD). Chron Respir Dis 2004;1:163-72.

36. Pryor JA, Webber BA, Bethune D, et al. Physiotherapy techniques. In: Pryor JA, Prasad SA, eds. Physiotherapy for respiratory and cardiac problems. Edinburgh: Churchill Livingstone, 2002:161-242.

37. Bott J. Physiotherapy. In: Morgan MDL, Singh SJ, eds. Practical pulmonary rehabilitation. London: Chapman and Hall, 1997:155-78.

38. Jones AY, Dean E, Chow CC. Comparison of the oxygen cost of breathing exercises and spontaneous breathing in patients with stable chronic obstructive pulmonary disease. Phys Ther 2003;83:424-31.

39. Vitacca M, Clini E, Bianchi L, et al. Acute effects of deep diaphragmatic breathing in COPD patients with chronic respiratory insufficiency. Eur Respir J 1998;11:408-15.

40. Gosselink RA, Wagenaar RC, Rijswijk H, et al. Diaphragmatic breathing reduces efficiency of breathing in patients with chronic obstructive pulmonary disease. Am J Respir Crit Care Med 1995;151:1136-42.

41. Garrod R, Dallimore K, Cook J, et al. An evaluation of the acute impact of pursed lips breathing on walking distance in nonspontaneous pursed lips breathing chronic obstructive pulmonary disease patients. Chron Respir Dis 2005;2:67-72.

42. Motley HL. The effects of slow deep breathing on the blood gas exchange in emphysema. Am Rev Respir Dis 1963;88:484-92.

43. Grassino A, Bellemare F, Laporta D. Diaphragm fatigue and the strategy of breathing in COPD. Chest 1984;85(Suppl):51S-4S 
44. Collins EG, Fehr L, Bammert C, et al. Effect of ventilation-feedback training on endurance and perceived breathlessness during constant work-rate leg-cycle exercise in patients with COPD. J Rehabil Res Dev 2003;40:35-44.

45. Collins EG, Langbein WE, Fehr $L$, et al. Can ventilation-feedback training augment exercise tolerance in patients with chronic obstructive pulmonary disease? Am J Respir Crit Care Med 2008;177:844-52.

46. Ries AL, Bauldoff GS, Carlin BW, et al. Pulmonary rehabilitation: joint ACCP/AACVPR evidence-based clinical practice guidelines. Chest 2007;131(Suppl)4S-42S.

47. Lacasse $\mathbf{Y}$, Goldstein R, Lasserson TJ, et al. Pulmonary rehabilitation for chronic obstructive pulmonary disease. Cochrane Database Syst Rev 2006; (4):CD003793

48. Singh SJ, Morgan MD, Scott S, et al. Development of a shuttle walking test of disability in patients with chronic airways obstruction. Thorax 1992;47:1019-24.

49. Revill SM, Morgan MD, Singh SJ, et al. The endurance shuttle walk: a new field test for the assessment of endurance capacity in chronic obstructive pulmonary disease. Thorax 1999:54:213-22.

50. Sciurba F, Criner GJ, Lee SM, et al. Six-minute walk distance in chronic obstructive pulmonary disease: reproducibility and effect of walking course layout and length. Am J Respir Crit Care Med 2003;167:1522-7.

51. Gray F, Smith A, Britton M, et al. Is a practice incremental shuttle walk test really necessary? Thorax 2006:61:ii57-133.

52. Lang $\mathbf{P}$, Dyer F, Bott J. Is a practice Incremental Shuttle Walking Test (ISWT) necessary for all patients? Eur Respir J 2008;32:4113.

53. Man WD, Polkey Ml, Donaldson N, et al. Community pulmonary rehabilitation after hospitalisation for acute exacerbations of chronic obstructive pulmonary disease: randomised controlled study. BMJ 2004;329:1209.

54. Murphy N, Bell C, Costello RW. Extending a home from hospital care programme for COPD exacerbations to include pulmonary rehabilitation. Respir Med 2005;99:1297-302.

55. Puhan MA, Scharplatz M, Troosters T, et al. Respiratory rehabilitation after acute exacerbation of COPD may reduce risk for readmission and mortality — a systematic review. Respir Res 2005;6:54

56. Bond JC, Vincent EE, Prime $\mathrm{H}$. In-patient pulmonary rehabilitation during acute exacerbation of chronic obstructive pulmonary disease: immediate effects on health status and exercise capacity. Thorax 2006;61:S68.

57. Smith K, Cook D, Guyatt GH, et al. Respiratory muscle training in chronic airflow limitation: a meta-analysis. Am Rev Respir Dis 1992;145:533-9.

58. Dekhuijzen PN, Folgering HT, van Herwaarden CL. Target-flow inspiratory muscle training during pulmonary rehabilitation in patients with COPD. Chest 1991;99:128-33.

59. Sturdy G, Hillman D, Green D, et al. Feasibility of high-intensity, interval-based respiratory muscle training in COPD. Chest 2003;123:142-50.

60. Sanchez-Riera H, Montemayor RT, Ortega RF, et al. Inspiratory muscle training in patients with COPD: effect on dyspnea, exercise performance, and quality of life. Chest 2001;120:748-56

61. Wanke T, Formanek D, Lahrmann $\mathrm{H}$, et al. Effects of combined inspiratory muscle and cycle ergometer training on exercise performance in patients with COPD. Eur Respir J 1994; 7:2205-11

62. Preusser BA, Winningham ML, Clanton TL. High- vs low-intensity inspiratory muscle interval training in patients with COPD. Chest 1994:106:110-7.

63. Lisboa C, Villafranca C, Leiva A, et al. Inspiratory muscle training in chronic airflow limitation: effect on exercise performance. Eur Respir J 1997:10:537-42.

64. Covey MK, Larson JL, Wirtz SE, et al. High-intensity inspiratory muscle training in patients with chronic obstructive pulmonary disease and severely reduced function. J Cardiopulm Rehabil 2001:21:231-240.

65. Berry MJ, Adair NE, Sevensky KS, et al. Inspiratory muscle training and wholebody reconditioning in chronic obstructive pulmonary disease. Am J Respir Crit Care Med 1996;153:1812-6.

66. Larson JL, Covey MK, Wirtz SE, et al. Cycle ergometer and inspiratory muscle training in chronic obstructive pulmonary disease. Am J Respir Crit Care Med 1999:160:500-7.

67. Lotters F, Van Tol B, Kwakkel G, et al. Effects of controlled inspiratory muscle training in patients with COPD: a meta-analysis. Eur Respir $J$ 2002;20:570-6.

68. O'Brien K, Geddes EL, Reid WD, et al. Inspiratory muscle training compared with other rehabilitation interventions in chronic obstructive pulmonary disease: a systematic review update. J Cardiopulm Rehabil Prev 2008;28:128-41.

69. Lisboa C, Munoz V, Beroiza T, et al. Inspiratory muscle training in chronic airflow limitation: comparison of two different training loads with a threshold device. Eur Respir J 1994:7:1266-74.

70. Kim MJ, Larson JL, Covey MK, et al. Inspiratory muscle training in patients with chronic obstructive pulmonary disease. Nurs Res 1993:42:356-62.

71. Harver A, Daubenspeck JA, Mahler DA. Targeted inspiratory muscle training improves respiratory muscle function and reduces dyspnea in patients with chronic obstructive pulmonary disease. Ann Intern Med 1989:111:117-24.

72. Larson JL, Kim MJ, Sharp JT, et al. Inspiratory muscle training with a pressure threshold breathing device in patients with chronic obstructive pulmonary disease. Am Rev Respir Dis 1988:138:689-96.

73. Weiner $\mathbf{P}$, Magadle $\mathrm{R}$, Beckerman $\mathrm{M}$, et al. Maintenance of inspiratory muscle training in COPD patients: one year follow-up. Eur Respir J 2004:23:61-5.

74. Crowe J, Reid WD, Geddes EL, et al. Inspiratory muscle training compared with other rehabilitation interventions in adults with chronic obstructive pulmonary disease: a systematic literature review and meta-analysis. COPD 2005:2:319-29.

75. Gaskell DV. Chronic bronchitis, emphysema and asthma. In: Downie PA, ed. Cash's textbook of chest, heart and vascular disorders for physiotherapists. London: Faber \& Faber, 1979:231-48
76. British Thoracic Society Standards of Care Committee. Non-invasive ventilation in acute respiratory failure. Thorax 2002:57:192-211.

77. Keenan SP, Kernerman PD, Cook DJ, et al. Effect of noninvasive positive pressure ventilation on mortality in patients admitted with acute respiratory failure: a metaanalysis. Crit Care Med 1997;25:1685-2

78. Ram FS, Picot J, Lightowler J, et al. Non-invasive positive pressure ventilation for treatment of respiratory failure due to exacerbations of chronic obstructive pulmonary disease. Cochrane Database Syst Rev 2004;(3):CD004104.

79. Wijkstra PJ, Lacasse Y, Guyatt GH, et al. Nocturnal non-invasive positive pressure ventilation for stable chronic obstructive pulmonary disease. Cochrane Database Syst Rev 2002;(2):CD002878.

80. Sukumalchantra Y, Park SS, Williams MH Jr. The effect of intermittent positive pressure breathing (IPPB) in acute ventilatory failure. Am Rev Respir Dis 1965; $92: 885-93$

81. Anthonisen NR, Hodgkin JE, Hopewell PC, et al. IPPB in COPD. Chest 1984:6:341-2

82. Lefcoe N, Carter P. Intermittent positive-pressure breathing in chronic obstructive pulmonary disease. Can Med Assoc J 1970;103:279-81.

83. Emirgil C, Sobol BJ, Norman J, et al. A study of the long-term effect of therapy in chronic obstructive pulmonary disease. Am J Med 1969;47:367-77.

84. IPPB Trial Group. Intermittent positive pressure breathing therapy of chronic obstructive pulmonary disease_a clinical trial. Ann Intern Med 1983;99:612-20.

85. Ayres SM, Kozam RL, Lukas DS. The effects of intermittent positive pressure breathing on intrathoracic pressure, pulmonary mechanics, and the work of breathing. Am Rev Respir Dis 1963;87:370-9.

86. Torres G, Lyons HA, Emerson P. The effects of intermittent positive pressure breathing on the intrapulmonary distribution of inspired air. Am J Med 1960;29:946-54.

87. Emmanuel GE, Smith WM, Briscoe WA. The effect of intermittent positive pressure breathing and voluntary hyperventilation upon the distribution of ventilation and pulmonary blood flow to the lung in chronic obstructive lung disease. J Clin Invest 1966;45:1221-33

88. Cullen JH, Brum VC, Reidt WU. An evaluation of the ability of intermittent positive pressure breathing to produce effective hyperventilation in severe pulmonary emphysema. Am Rev Tuberc 1957;76:33-46

89. Fraimow W, Cathcart RT, Goodman E. The use of intermittent positive pressure breathing in the prevention of carbon dixide narcosis associated with oxygen therapy. Am Rev Respir Dis 1960;81:815-22.

90. Israel RH, Poe RH. Insidious onset of acute alveolar hypoventilation following intermittent positive pressure breathing (IPPB). Respiration 1981;41:199-201.

91. Starke ID, Webber BA, Branthwaite MA. IPPB and hypercapnia in respiratory failure: the effect of different concentrations of inspired oxygen on arterial blood gas tensions. Anaesthesia 1979;34:283-7.

92. British Thoracic Society. Emergency oxygen use in adult patients. http://www. brit-thoracic.org.uk (accessed March 2009)

93. British Thoracic Society and Department of Health. Clinical component for the home oxygen service in England and Wales. http://www.brit-thoracic.org.uk/Portals/ 0/Clinical\%20Information/ Home\%200xygen\%20Service/clinical\%20adultoxygenjan06. pdf (accessed March 2009).

94. Crisafulli E, Costi S, De BF, et al. Effects of a walking aid in COPD patients receiving oxygen therapy. Chest 2007;131:1068-74.

95. Hess DR. The evidence for secretion clearance techniques. Cardiopulm Phys Ther 2002;13:7-22.

96. Hess DR. The evidence for secretion clearance techniques. Respir Care 2001;46:1276-93.

97. Savci S, Ince DI, Arikan H. A comparison of autogenic drainage and the active cycle of breathing techniques in patients with chronic obstructive pulmonary diseases. J Cardiopulm Rehabil 2000:20:37-43.

98. van der Schans CP, Goldstein RS, Bach JR. Airway secretion management and oxygen therapy. Phys Med Rehabil Clin N Am 1996:7:277-98.

99. Clarke SW. Management of mucus hypersecretion. Eur J Respir Dis Supp/ 1987; 153:136-44.

100. Mortensen J, Jensen C, Groth S, et al. The effect of forced expirations on mucociliary clearance in patients with chronic bronchitis and in healthy subjects. Clin Physiol 1991;11:439-50.

101. Bellone A, Lascioli R, Raschi S, et al. Chest physical therapy in patients with acute exacerbation of chronic bronchitis: effectiveness of three methods. Arch Phys Med Rehabil 2000;81:558-60.

102. Button BM, Sherburn M, Chase J, et al. Pelvic floor muscle function in women with chronic lung disease (cystic fibrosis and COPD) versus controls: relationship to urinary incontinence. Pediatr Pulmonol 2005:40:a368.

103. Cornacchia $\mathbf{M}$, Zenorini $A$, Perobelli $\mathbf{S}$, et al. Prevalence of urinary incontinence in women with cystic fibrosis. BJU Int 2001;88:44-8.

104. White D, Stiller K, Roney F. The prevalence and severity of symptoms of incontinence in adult cystic fibrosis patients. Physiother Theory Pract 2000;16:35-42.

105. Orr A, McVean RJ, Webb AK, et al. Questionnaire survey of urinary incontinence in women with cystic fibrosis. BMJ 2001:322:1521.

106. Moran F, Bradley JM, Boyle L, et al. Incontinence in adult females with cystic fibrosis: a Northern Ireland survey. Int J Clin Pract 2003:57:182-3.

107. Button BM, Sherburn M, Chase J, et al. Effect of a three months physiotherapeutic intervention on incontinence in women with chronic cough related to cystic fibrosis and COPD. Pediatr Pulmonol 2005:40:a369.

108. Miller JM, Ashton-Miller JA, DeLancey JO. A pelvic muscle precontraction can reduce cough-related urine loss in selected women with mild SUI. J Am Geriatr Soc $1998 \cdot \mathbf{4 6}: 870-4$ 
109. British Thoracic Society. Guidelines for management of asthma in adults: IChronic persistent asthma. Statement by the British Thoracic Society, Research Unit of the Royal College of Physicians of London, King's Fund Centre, National Asthma Campaign. BMJ 1990;301:651-3.

110. British Thoracic Society. Guidelines for management of asthma in adults: ॥Acute severe asthma. Statement by the British Thoracic Society, Research Unit of the Royal College of Physicians of London, King's Fund Centre, National Asthma Campaign. BMJ 1990;301:797-800

111. British Thoracic Society, Scottish Intercollegiate Guidelines Network British Guideline on the Management of Asthma. Thorax 2003;58.

112. British Thoracic Society. Guidelines on the management of asthma. Statement by the British Thoracic Society, the British Paediatric Association, the Research Unit of the Royal College of Physicians of London, the King's Fund Centre, the National Asthma Campaign, the Royal College of General Practitioners, the General Practitioners in Asthma Group, the British Association of Accident and Emergency Medicine, and the British Paediatric Respiratory Group. Thorax 1993;48:S1-24.

113. British Thoracic Society. The British Guidelines on Asthma Management 1995 Review and Position Statement. Thorax 1997;52:1S-20.

114. British Thoracic Society. British guideline on the management of asthma. Thorax 2008;63(Suppl IV):iv1-121.

115. Pryor JA, Prasad SA. Physiotherapy for respiratory and cardiac problems. 3rd edn. Edinburgh: Churchill Livingstone, 2002

116. Bruton A, Thomas M. Breathing therapies and bronchodilator use in asthma. Thorax 2006;61:643-5.

117. Holloway E, Ram FS. Breathing exercises for asthma. Cochrane Database Syst Rev 2004: 1):CD001277.

118. Ernst E. Breathing techniques_-adjunctive treatment modalities for asthma? A systematic review. Eur Respir J 2000;15:969-72.

119. Slader CA, Reddel HK, Spencer LM, et al. Double blind randomised controlled trial of two different breathing techniques in the management of asthma. Thorax 2006;61:651-6.

120. Holloway EA, West R. Integrated breathing and relaxation training (the Papworth Method) for adults with asthma in primary care: a randomised controlled trial. Thorax 2007:62:1039-42.

121. Cowie RL, Conley DP, Underwood MF, et al. A randomised controlled trial of the Buteyko technique as an adjunct to conventional management of asthma. Respir Med 2008:726-32.

122. Thomas M, McKinley RK, Mellor S, et al. Breathing exercises for asthma: a randomised controlled trial. Thorax 2009:64:55-61.

123. Cooper S, Oborne J, Newton S, et al. Effect of two breathing exercises (Buteyko and pranayama) in asthma: a randomised controlled trial. Thorax 2003:58:674-9.

124. Bowler SD, Green A, Mitchell CA. Buteyko breathing techniques in asthma: a blinded randomised controlled trial. Med J Aust 1998;169:575-8.

125. Bruton A, Lewith GT. The Buteyko breathing technique for asthma: a review. Complement Ther Med 2005;13:41-6.

126. Bishop A, Rawle M, Bruton A. The use of mouth taping in people with asthma: a pilot study examining the effects on end-tidal carbon dioxide levels. Physiotherapy 2007:93:129-36.

127. Ram FS, Robinson SM, Black PN, et al. Physical training for asthma. Cochrane Database Syst Rev 2005; (4):CD001116

128. Cochrane LM, Clark CJ. Benefits and problems of a physical training programme for asthmatic patients. Thorax 1990;45:345-51.

129. Cambach W, Chadwick-Straver RV, Wagenaar RC, et al. The effects of a community-based pulmonary rehabilitation programme on exercise tolerance and quality of life: a randomized controlled trial. Eur Respir J 1997;10:104-13.

130. Cox NJ, Hendricks JC, Binkhorst RA, et al. A pulmonary rehabilitation program for patients with asthma and mild chronic obstructive pulmonary diseases (COPD). Lung 1993;171:235-44

131. Emtner M, Herala M, Stalenheim G. High-intensity physical training in adults with asthma. A 10-week rehabilitation program. Chest 1996;109:323-30.

132. Emtner M, Finne M, Stalenheim G. A 3-year follow-up of asthmatic patients participating in a 10-week rehabilitation program with emphasis on physical training. Arch Phys Med Rehabil 1998;79:539-44.

133. Emtner M, Hedin A, Stalenheim G. Asthmatic patients' views of a comprehensive asthma rehabilitation programme: a three-year follow-up. Physiother Res Int 1998;3:175-93.

134. Hallstrand TS, Bates PW, Schoene RB. Aerobic conditioning in mild asthma decreases the hyperpnea of exercise and improves exercise and ventilatory capacity. Chest 2000;118:1460-9.

135. Robinson DM, Egglestone DM, Hill PM, et al. Effects of a physical conditioning programme on asthmatic patients. N Z Med J 1992;105:253-6.

136. Emtner M. Physiotherapy and intensive physical training in rehabilitation of adults with asthma. Phys Ther Rev 1999;4:229-40.

137. Krumholz RA. Rehabilitation on the chronic asthmatic. Compr Ther 1978;4:38-41

138. Ambrosino N, Vitacca M, Rampulla C. Standards for rehabilitative strategies in respiratory diseases. Monaldi Arch Chest Dis 1995;50:293-318.

139. Wolf SI. Rehabilitation of asthmatic patients. Motivating your patients to improve their life-style. Postgrad Med 1991;90:93-6.

140. Weiner P, Berar-Yanay N, Davidovich A, et al. Specific inspiratory muscle training in patients with mild asthma with high consumption of inhaled beta(2)-agonists. Chest 2000;117:722-7.
141. Weiner $\mathbf{P}$, Magadle R, Massarwa F, et al. Influence of gender and inspiratory muscle training on the perception of dyspnea in patients with asthma. Chest 2002;122:197-201.

142. Barnabé V, Saraiva B, Stelmach R, et al. Chest physiotherapy does not induce bronchospasm in stable asthma. Physiotherapy 2003;89:714-9

143. Christensson P, Cimbritz H, Arborelius M Jr, et al. Effect of salbutamol with IPPB and physiotherapy for three days. Scand J Respir Dis Supp/ 1977:101:109-12.

144. Hondras MA, Linde K, Jones AP. Manual therapy for asthma. Cochrane Database Syst Rev 2005:(2):CD001002.

145. Swift GL, Rainer T, Saran R, et al. Use of Flutter VRP1 in the management of patients with steroid-dependent asthma. Respiration 1994;61:126-9.

146. Aitken ML, Vincent JM, Pierson DJ. Effects of pulmonary function of oral high frequency oscillation in normal and asthmatic subjects. Respir Med 1992:86:211-4.

147. Tsai CF, Tsai JJ. Effectiveness of a positive expiratory pressure device in conjunction with beta2-agonist nebulization therapy for bronchial asthma. J Microbiol Immunol Infect 2001;34:92-6.

148. Lehrer PM, Vaschillo E, Vaschillo B, et al. Biofeedback treatment for asthma. Chest 2004;126:352-61.

149. Lum LC. Symptoms and signs of hyperventilation. In: Timmons B, Ley R, eds. Behavioural and psychological approaches in breathing disorders. London: Plenum Press, 1994:113-23

150. Thomas M, McKinley RK, Freeman E, et al. Breathing retraining for dysfunctional breathing in asthma: a randomised controlled trial. Thorax 2003;58:110-5.

151. Compernolle T, Hoogduin K, Joele L. Diagnosis and treatment of the hyperventilation syndrome. Psychosomatics 1979;20:612-25.

152. Kraft AR, Hoogduin CA. The hyperventilation syndrome. A pilot study on the effectiveness of treatment. Br J Psychiatry 1984;145:538-42.

153. Grossman $\mathbf{P}$, de Swart JC, Defares PB. A controlled study of a breathing therapy for treatment of hyperventilation syndrome. J Psychosom Res 1985;29:49-58.

154. Cluff RA. Chronic hyperventilation and its treatment by physiotherapy. Physiotherapy 1985; 11:301-5.

155. DeGuire S, Gevirtz R, Kawahara Y, et al. Hyperventilation syndrome and the assessment of treatment for functional cardiac symptoms. Am J Cardiol 1992;70:673-7.

156. DeGuire S, Gevirtz R, Hawkinson D, et al. Breathing retraining: a three-year followup study of treatment for hyperventilation syndrome and associated functional cardiac symptoms. Biofeedback Self Regul 1996;21:191-8.

157. Han JN, Stegan K, De Valck C, et al. Influence of breathing therapy on complaints, anxiety and breathing pattern in patients with hyperventilation syndrome and anxiety disorders. J Psychosom Res 1966:41:483-91.

158. Newsham KR, Klaben BK, Miller VJ, et al. Paradoxical vocal-cord dysfunction: management in athletes. J Athletic Training 2002;37:325-8.

159. Bradley J, Moran F. Physical training for cystic fibrosis. Cochrane Database Syst Rev 2002;(1):CD002768.

160. Dodge JA, Lewis PA, Stanton M, et al. Cystic fibrosis mortality and survival in the UK: 1947-2003. Eur Respir J 2007:29:522-6.

161. World Health Organization. Adherence to long-term therapies: evidence for action. Geneva: World Health Organization, 2003.

162. Kettler LJ, Sawyer SM, Winefield HR, et al. Determinants of adherence in adults with cystic fibrosis. Thorax 2002;57:459-64.

163. Dodd ME, Webb AK. Understanding non-compliance with treatment in adults with cystic fibrosis. J $R$ Soc Med 2000;93(Suppl 38):2-8.

164. Conway SP, Pond MN, Hamnett T, et al. Compliance with treatment in adult patients with cystic fibrosis. Thorax 1996;51:29-33.

165. American College of Sports Medicine. American College of Sports Medicine Guidelines for exercise testing and prescription. 7th edn. Lippincott Williams \& Wilkins, 2005

166. van der Schans CP, Prasad A, Main E. Chest physiotherapy compared to no chest physiotherapy for cystic fibrosis. Cochrane Database Syst Rev 2000;(2):CD001401.

167. Main E, Prasad A, van der Schans CP. Conventional chest physiotherapy compared to other airway clearance techniques for cystic fibrosis. Cochrane Database Syst Rev 2005; (1):CD002011.

168. Robinson P. Cystic fibrosis. Thorax 2001:56:237-41.

169. Pryor JA, Webber BA, Hodson ME, et al. Evaluation of the forced expiration technique as an adjunct to postural drainage in treatment of cystic fibrosis. Br Med J 1979:2:417-8.

170. Williams MT, Parsons DW, Frick RA, et al. Acute respiratory infection in patients with cystic fibrosis with mild pulmonary impairment: comparison of two physiotherapy regimens. Aust J Physiother 2001;47:227-36.

171. Pryor JA, Webber BA, Hodson ME, et al. The Flutter VRP1 as an adjunct to chest physiotherapy in cystic fibrosis. Respir Med 1994:88:677-81.

172. Pike SE, Pryor JA, Machin AC, et al. Comparison of Flutter VRP1 and forced expirations (FE) with the active cycle of breathing techniques (ACBT) in subjects with cystic fibrosis (CF). Neth J Med 1999;54:S55-6.

173. Hofmeyr JL, Webber BA, Hodson ME. Evaluation of positive expiratory pressure as an adjunct to chest physiotherapy in the treatment of cystic fibrosis. Thorax 1986:41:951-4

174. Miller S, Hall DO, Clayton CB, et al. Chest physiotherapy in cystic fibrosis: a comparative study of autogenic drainage and the active cycle of breathing techniques with postural drainage. Thorax 1995;50:165-9.

175. Pryor JA, Tannenbaum E, Cramer D, et al. A comparison of five airway clearance techniques in the treatment of people with cystic fibrosis. J Cyst Fibros 2006;:5:S76. 
176. Mcllwaine PM, Davidson AG, Wong LT, et al. The effect of chest physiotherapy by postural drainage and autogenic drainage on oxygen saturation in cystic fibrosis. Pediatr Pulmonol 1991;11:291.

177. Davidson AG, Wong LT, Pirie GE, et al. Long-term comparative trial of conventional percussion and drainage physiotherapy versus autogenic drainage in CF. Pediatr Pulmonol 1992:14:298.

178. Pfleger A, Theissl B, Oberwaldner B, et al. Self-administered chest physiotherapy in cystic fibrosis: a comparative study of high-pressure PEP and autogenic drainage. Lung 1992;170:323-30.

179. App EM, Kieselmann R, Reinhardt D, et al. Sputum rheology changes in cystic fibrosis lung disease following two different types of physiotherapy: Flutter vs autogenic drainage. Chest 1998;114:171-7.

180. Lannefors L, Wollmer P. Mucus clearance with three chest physiotherapy regimes in cystic fibrosis: a comparison between postural drainage, PEP and physical exercise. Eur Respir J 1992;5:748-53.

181. Elkins MR, Jones A, van der Schans CP. Positive expiratory pressure physiotherapy for airway clearance in people with cystic fibrosis. Cochrane Database Syst Rev 2006;(2):CD003147

182. Homnick DN, Anderson K, Marks JH. Comparison of the Flutter device to standard chest physiotherapy in hospitalized patients with cystic fibrosis: a pilot study. Chest 1998;114:993-7.

183. Gondor M, Nixon PA, Mutich R, et al. Comparison of Flutter device and chest physical therapy in the treatment of cystic fibrosis pulmonary exacerbation. Pediatr Pulmonol 1999;28:255-60

184. Oberwaldner B, Evans JC, Zach MS. Forced expirations against a variable resistance: a new chest physiotherapy method in cystic fibrosis. Pediatr Pulmonol 1986;2:358-67.

185. Homnick DN, White F, de Castro C. Comparison of effects of an intrapulmonary percussive ventilator to standard aerosol and chest physiotherapy in treatment of cystic fibrosis. Pediatr Pulmonol 1995:20:50-5.

186. Marks JH, Hare KL, Saunders RA, et al. Pulmonary function and sputum production in patients with cystic fibrosis: a pilot study comparing the PercussiveTech $\mathrm{HF}$ device and standard chest physiotherapy. Chest 2004:125:1507-11.

187. Cecins NM, Jenkins SC, Pengelley J, et al. The active cycle of breathing techniques - to tip or not to tip? Respir Med 1999;93:660-5.

188. McCarren B, Alison JA. Physiological effects of vibration in subjects with cystic fibrosis. Eur Respir J 2006;27:1204-9.

189. McCarren B, Alison JA, Herbert RD. Manual vibration increases expiratory flow rate via increased intrapleural pressure in healthy adults: an experimental study. Aust J Physiother 2006;52:267-71.

190. Oermann CM, Sockrider MM, Giles D, et al. Comparison of high-frequency chest wall oscillation and oscillating positive expiratory pressure in the home management of cystic fibrosis: a pilot study. Pediatr Pulmonol 2001:32:372-7.

191. Arens R, Gozal D, Omlin KJ, et al. Comparison of high frequency chest compression and conventional chest physiotherapy in hospitalized patients with cystic fibrosis. Am J Respir Crit Care Med 1994;150:1154-7.

192. Osman LP, Roughton M, Hodson ME, et al. High frequency chest wall oscillation in cystic fibrosis. J Cyst Fibros 2008;7:S73.

193. Hartsell MB. Chest physiotherapy and mechanical vibration. J Pediatr Nurs 1987:2:135-7.

194. Lapin CD. Assisted cough devices. Pediatr Pulmonol Supp/ 2004;26:149-51

195. Agent $\mathbf{P}$, Chatwin M, Ross E. Mechanical in-exsufflation in adults with cystic fibrosis-a physiological investigation. J Cyst Fibros 2003;2:A237.

196. Salh W, Bilton D, Dodd M, et al. Effect of exercise and physiotherapy in aiding sputum expectoration in adults with cystic fibrosis. Thorax 1989;44:1006-8.

197. Bilton D, Dodd ME, Abbot JV, et al. The benefits of exercise combined with physiotherapy in the treatment of adults with cystic fibrosis. Respir Med 1992;86:507-11.

198. Baldwin DR, Hill AL, Peckham DG, et al. Effect of addition of exercise to chest physiotherapy on sputum expectoration and lung function in adults with cystic fibrosis. Respir Med 1994:88:49-53.

199. American Association for Respiratory Care. Nasotracheal suction-2004 revision \& update. Respir Care 2004;49:1080-4.

200. Chatham K, lonescu AA, Nixon LS, et al. A short-term comparison of two methods of sputum expectoration in cystic fibrosis. Eur Respir J 2004;23:435-9.

201. Enright S, Chatham $\mathrm{K}$, Ionescu $\mathrm{AA}$, et al. Inspiratory muscle training improves lung function and exercise capacity in adults with cystic fibrosis. Chest 2004;126:405-11.

202. Asher MI, Pardy RL, Coates AL, et al. The effects of inspiratory muscle training in patients with cystic fibrosis. Am Rev Respir Dis 1982:126:855-859.

203. Moran F, Bradley J, Piper AJ, et al. Non-invasive ventilation for cystic fibrosis. Cochrane Database Syst Rev 2009;(1).CD002769.

204. Holland AE, Denehy L, Ntoumenopoulos G, et al. Non-invasive ventilation assists chest physiotherapy in adults with acute exacerbations of cystic fibrosis. Thorax 2003:58:880-4.

205. Sorenson HM, Shelledy DC. AARC clinical practice guideline. Intermittent positive pressure breathing —2003 revision \& update. Respir Care 2003:48:540-6.

206. Henke KG, Regnis JA, Bye PT. Benefits of continuous positive airway pressure during exercise in cystic fibrosis and relationship to disease severity. Am Rev Respir Dis 1993;148:1272-6.

207. Dodd ME, Haworth CS, Webb AK. A practical approach to oxygen therapy in cystic fibrosis. J R Soc Med 1998;91(Suppl 34):30-9

208. Coates AL. Oxygen therapy, exercise, and cystic fibrosis. Chest 1992;101:2-4.
209. Marcus CL, Bader D, Stabile MW, et al. Supplemental oxygen and exercise performance in patients with cystic fibrosis with severe pulmonary disease. Chest 1992;101:52-7

210. Kellett F, Redfern J, Niven RM. Evaluation of nebulised hypertonic saline (7\%) as an adjunct to physiotherapy in patients with stable bronchiectasis. Respir Med 2005;99:27-31.

211. Patterson JE, Bradley JM, Hewitt 0, et al. Airway clearance in bronchiectasis: a randomized crossover trial of active cycle of breathing techniques versus Acapella. Respiration 2005; 72:239-42.

212. Wark PA, McDonald V, Jones AP. Nebulised hypertonic saline for cystic fibrosis Cochrane Database Syst Rev 2005;(3):CD001506.

213. Robinson M, Hemming AL, Regnis JA, et al. Effect of increasing doses of hypertonic saline on mucociliary clearance in patients with cystic fibrosis. Thorax 1997; 52:900-3.

214. Jones AP, Wallis CE, Kearney CE. Dornase alpha for cystic fibrosis. Cochrane Database Syst Rev 2003;(3):CD001127.

215. Dodd ME, Moorcroft AJ, Haworth CS, et al. The effect of rhDNase on exercise performance and gas trapping in adults with cystic fibrosis: a randomised controlled trial. 13th International Cystic Fibrosis Congress; June 4-8; Stockholm, Sweden. 2000.

216. Tattersall R, Walshaw MJ. Posture and cystic fibrosis. J R Soc Med 2003;96(Suppl 43):18-22.

217. Massery M. Musculoskeletal and neuromuscular interventions: a physical approach to cystic fibrosis. J R Soc Med 2005;98(Suppl 45):55-66.

218. Rose J, Gamble J, Schultz A, et al. Back pain and spinal deformity in cystic fibrosis Am J Dis Child 1987:141:1313-6.

219. Demry A, Ami S, Levi M, et al. Chest strength and mobility training: a new approach to airways clearance. J Cyst Fibros 2006:5:S82.

220. Dodd ME, Langman H. Urinary incontinence in cystic fibrosis. J R Soc Med 2005;98:28-36.

221. McVean RJ, Orr A, Webb AK, et al. Treatment of urinary incontinence in cystic fibrosis. J Cyst Fibros 2003;2:171-6.

222. Cystic Fibrosis Trust (UK) Infection Control Advisory Group. Consensus guidelines. http://www.cftrust.org.uk/aboutcf/publications/consensusdoc/.2004 (accessed October 2008)

223. Dweik R, Stoller JK. Obstructive lung disease: COPD, asthma, and related diseases. In: Scanlan CL, Wilkins RL, Stoller JK, eds. Egan's fundamentals of respiratory care. St Louis, MO: Mosby, 1999:441-62.

224. Wills $\mathbf{P}$, Greenstone M. Inhaled hyperosmolar agents for bronchiectasis. Cochrane Database Syst Rev 2006;(2):CD002996.

225. Pryor JA. Physical therapy for adults with bronchiectasis. Clin Pulm Med 2004:11:201-9.

226. O'Neill B, Bradley JM, McArdle N, et al. The current physiotherapy management of patients with bronchiectasis: a UK survey. Int J Clin Pract 2002;56:34-5.

227. Koulouris NG, Retsou S, Kosmas E, et al. Tidal expiratory flow limitation, dyspnoea and exercise capacity in patients with bilateral bronchiectasis. Eur Respir $J$ 2003:21:743-8.

228. Bradley J, Moran F, Greenstone M. Physical training for bronchiectasis. Cochrane Database Syst Rev 2002;(3):CD002166.

229. Newall C, Stockley RA, Hill SL. Exercise training and inspiratory muscle training in patients with bronchiectasis. Thorax 2005;60:943-8.

230. Sutton PP, Gemmell HG, Innes N, et al. Use of nebulised saline and nebulised terbutaline as an adjunct to chest physiotherapy. Thorax 1988:43:57-60.

231. Bateman JR, Newman SP, Daunt KM, et al. Is cough as effective as chest physiotherapy in the removal of excessive tracheobronchial secretions? Thorax 1981:36:683-7.

232. Jones AP, Rowe BH. Bronchopulmonary hygiene physical therapy for chronic obstructive pulmonary disease and bronchiectasis. Cochrane Database Syst Rev 1998;(4):CD000045.

233. Sheehan RE, Wells AU, Copley SJ, et al. A comparison of serial computed tomography and functional change in bronchiectasis. Eur Respir J 2002:20:581-7.

234. Dodd ME, Webb AK. Bronchiectasis, primary ciliary dyskinesia and cystic fibrosis In: Pryor JA, Prasad SA, eds. Physiotherapy for respiratory and cardiac problems. Edinburgh: Churchill Livingstone, 2002:581-617.

235. Sutton PP, Parker RA, Webber BA, et al. Assessment of the forced expiration technique, postural drainage and directed coughing in chest physiotherapy. Eur J Respir Dis 1983;64:62-8.

236. Eaton T, Young P, Zeng I, et al. A randomized evaluation of the acute efficacy, acceptability and tolerability of Flutter and active cycle of breathing with and without postural drainage in non-cystic fibrosis bronchiectasis. Chron Respir Dis 2007;4:23-30.

237. Currie DC, Munro C, Gaskell D, et al. Practice, problems and compliance with postural drainage: a survey of chronic sputum producers. $\mathrm{Br} \mathrm{J}$ Dis Chest 1986;80:249-53

238. Chen HC, Liu CY, Cheng HF, et al. Chest physiotherapy does not exacerbate gastroesophageal reflux in patients with chronic bronchitis and bronchiectasis. Changgeng Yi Xue Za Zhi 1998;21:409-14.

239. Gallon A. Evaluation of chest percussion in the treatment of patients with copious sputum production. Respir Med 1991;85:45-51

240. Sutton PP, Lopez-Vidriero MT, Pavia D, et al. Assessment of percussion, vibratoryshaking and breathing exercises in chest physiotherapy. Eur J Respir Dis 1985;66:147-52.

241. Mazzocco MC, Owens GR, Kirilloff LH, et al. Chest percussion and postural drainage in patients with bronchiectasis. Chest 1985:88:360-3. 
242. Thompson CS, Harrison S, Ashley J, et al. Randomised crossover study of the Flutter device and the active cycle of breathing technique in non-cystic fibrosis bronchiectasis. Thorax 2002;57:446-8.

243. Patterson JE, Bradley JM, Elborn JS. Airway clearance in bronchiectasis: a randomized crossover trial of active cycle of breathing techniques (incorporating postural drainage and vibration) versus test of incremental respiratory endurance. Chron Respir Dis 2004;1:127-30.

244. O'Connor C, Bridge P. Can the interrupter technique be used as an outcome measure for autogenic drainage in bronchiectatic patients? A pilot study. $J$ Assoc Chartered Physiother Resp Care 2005;37:29-34.

245. Valente AM, Gastaldi AC, Cravo SL, et al. The effect of two techniques on the characteristics and transport of sputum in patients with bronchiectasis: a pilot study. Physiotherapy 2004;90:158-64.

246. Nakamura S, Mikami M, Kawakami M, et al. Comparative evaluation of the Flutter and the Cornet in improving the cohesiveness of sputum from patients with bronchiectasis. Eur Respir J 1998;12:212S-3S

247. Tsang SMH, Jones AYM. Postural drainage or Flutter device in conjunction with breathing and coughing compared to breathing and coughing alone in improving secretion removal and lung function in patients with acute exacerbation of bronchiectasis: a pilot study. Hong Kong Physiother J 2003:21:29-36.

248. Hasani A, Pavia D, Agnew JE, et al. Regional lung clearance during cough and forced expiration technique (FET): effects of flow and viscoelasticity. Thorax 1994;49:557-61.

249. Conway JH, Fleming JS, Perring S, et al. Humidification as an adjunct to chest physiotherapy in aiding tracheo-bronchial clearance in patients with bronchiectasis. Respir Med 1992;86:109-14

250. Wills PJ, Hall RL, Chan W, et al. Sodium chloride increases the ciliary transportability of cystic fibrosis and bronchiectasis sputum on the mucus-depleted bovine trachea. J Clin Invest 1997;99:9-13

251. Schoeffel RE, Anderson SD, Altounyan RE. Bronchial hyperreactivity in response to inhalation of ultrasonically nebulised solutions of distilled water and saline. $B$ Med J (Clin Res Ed) 1981;283:1285-7.

252. Rodwell LT, Anderson SD. Airway responsiveness to hyperosmolar saline challenge in cystic fibrosis: a pilot study. Pediatr Pulmonol 1996;21:282-9.

253. Suri R, Wallis C, Bush A. Tolerability of nebulised hypertonic saline in children with cystic fibrosis. Pediatr Pulmonol 2000;30:306.

254. Foster S, Thomas HM III. Pulmonary rehabilitation in lung disease other than chronic obstructive pulmonary disease. Am Rev Respir Dis 1990;141:601-4.

255. Ando M, Mori A, Esaki $\mathrm{H}$, et al. The effect of pulmonary rehabilitation in patients with post-tuberculosis lung disorder. Chest 2003:123:1988-95.

256. Ferreira G, Feuerman M, Spiegler P. Results of an 8-week, outpatient pulmonary rehabilitation program on patients with and without chronic obstructive pulmonary disease. J Cardiopulm Rehabil 2006:26:54-60.

257. Dix K, Daly C, Garrod R, et al. Pulmonary rehabilitation in restrictive lung disease. Thorax 2002; 57:iii48-94

258. Congleton J, Bott J, Hindell A, et al. Comparison of outcome of pulmonary rehabilitation in obstructive lung disease, interstitial lung disease and chest wall disease. Thorax 1997:52:A11.

259. Mundy LM, Leet TL, Darst K, et al. Early mobilization of patients hospitalized with community-acquired pneumonia. Chest 2003;124:883-9.

260. Britton S, Bejstedt M, Vedin L. Chest physiotherapy in primary pneumonia. $\mathrm{Br}$ Med J (Clin Res Ed) 1985;290:1703-4.

261. Tydeman D. An investigation into the effectiveness of physiotherapy in the treatment of patients with community acquired pneumonia. Physiother Theory Pract 1989:5:75-8.

262. Bjorkqvist M, Wiberg B, Bodin L, et al. Bottle-blowing in hospital-treated patients with community-acquired pneumonia. Scand J Infect Dis 1997;29:77-82.

263. Brett A, Sinclair DG. Use of continuous positive airway pressure in the management of community acquired pneumonia. Thorax 1993;48:1280-1.

264. Prevedoros HP, Lee RP, Marriot D. CPAP, effective respiratory support in patients with AIDS-related Pneumocystis carinii pneumonia. Anaesth Intensive Care 1991;19:561-6.

265. Confalonieri M, Potena A, Carbone G, et al. Acute respiratory failure in patients with severe community-acquired pneumonia. A prospective randomized evaluation of noninvasive ventilation. Am J Respir Crit Care Med 1999;160:1585-91.

266. Graham WG, Bradley DA. Efficacy of chest physiotherapy and intermittent positivepressure breathing in the resolution of pneumonia. N Engl J Med 1978;299:624-7.

267. Kesten S, Garfinkel SK, Wright T, et al. Impaired exercise capacity in adults with moderate scoliosis. Chest 1991:99:663-6.

268. Weiss HR. The effect of an exercise program on vital capacity and rib mobility in patients with idiopathic scoliosis. Spine 1991:16:88-93.

269. Hornstein S, Inman S, Ledsome JR. Ventilatory muscle training in kyphoscoliosis. Spine 1987; 12:859-63.

270. Jones DJ, Paul EA, Bell JH, et al. Ambulatory oxygen therapy in stable kyphoscoliosis. Eur Respir J 1995;8:819-23.

271. Lindh $\mathbf{M}$, Nachemson $\mathbf{A}$. The effect of breathing exercises on the vital capacity in patients with scoliosis treated by surgical correction with the Harrington technique. Scand J Rehabil Med 1970;2:1-6.

272. De Troyer A, Borenstein S, Cordier R. Analysis of lung volume restriction in patients with respiratory muscle weakness. Thorax 1980;35:603-10.

273. Forner JV. Lung volumes and mechanics of breathing in tetraplegics. Paraplegia 1980:18:258-66.
274. Bake B, Fugl-Meyer AR, Grimby G. Breathing patterns and regional ventilation distribution in tetraplegic patients and in normal subjects. Clin Sci 1972;42:117-28.

275. Boaventura CM, Gastaldi AC, Silveira JM, et al. Effect of an abdominal binder on the efficacy of respiratory muscles in seated and supine tetraplegic patients. Physiotherapy 2003;89:290-5

276. Estenne M, Van Muylwm A, Gorini M, et al. Effects of abdominal strapping on forced expiration in tetraplegic patients. Am J Respir Crit Care Med 1998:157:95-8.

277. De Troyer A, Deisser P. The effects of intermittent positive pressure breathing on patients with respiratory muscle weakness. Am Rev Respir Dis 1981;124:132-7.

278. Lanig IS, Peterson WP. The respiratory system in spinal cord injury. Phys Med Rehabil Clin N Am 2000;11:29-43.

279. Estenne M, Pinet C, De Troyer A. Abdominal muscle strength in patients with tetraplegia. Am J Respir Crit Care Med 2000;161:707-12.

280. Gross D, Ladd HW, Riley EJ, et al. The effect of training on strength and endurance of the diaphragm in quadriplegia. Am J Med 1980:68:27-35.

281. Bach JR. Update and perspective on noninvasive respiratory muscle aids. Part 2 the expiratory aids. Chest 1994;105:1538-44.

282. Bach JR, Smith WH, Michaels J, et al. Airway secretion clearance by mechanica exsufflation for post-poliomyelitis ventilator-assisted individuals. Arch Phys Med Rehabil 1993:74:170-7.

283. De Troyer A, Estenne M. The expiratory muscles in tetraplegia. Paraplegia 1991;29:359-63

284. Bhaskar KR, Brown R, O'Sullivan DD, et al. Bronchial mucus hypersecretion in acute quadriplegia. Macromolecular yields and glycoconjugate composition. Am Rev Respir Dis 1991;143:640-8.

285. Van Houtte S, Vanlandewijck Y, Gosselink R. Respiratory muscle training in persons with spinal cord injury: a systematic review. Respir Med 2006;100:1886-95.

286. Morgan MDL, Silver RJ, Williams SJ. The respiratory system of the spinal cord patient. In: Bloch RF, Basbaum M, eds. Management of spinal cord injuries. Baltimore: Williams and Wilkins, 1986

287. Derrickson J, Ciesla N, Simpson N, et al. A comparison of two breathing exercise programs for patients with quadriplegia. Phys Ther 1992;72:763-9.

288. Consortium for Spinal Cord Medicine. Respiratory management following spinal cord injury: a clinical practice guideline for health-care professionals. J Spinal Cord Med 2005;28:259-93.

289. Roth EJ, Nussbaum SB, Berkowitz M, et al. Pulmonary function testing in spinal cord injury: correlation with vital capacity. Paraplegia 1995:33:454-7.

290. Green M, Moxham J. Respiratory muscles. In: Fenley DC, Petty TL, eds. Recent advances in respiratory medicine. Edinburgh: Churchill Livingstone, 1983:1-20.

291. Linn WS, Adkins RH, Gong $\mathrm{H}$ Jr, et al. Pulmonary function in chronic spinal cord injury: a cross-sectional survey of 222 southern California adult outpatients. Arch Phys Med Rehabil 2000;81:757-63.

292. Ali J, Qi W. Pulmonary function and posture in traumatic quadriplegia. J Trauma 1995:39:334-7.

293. Goldman AL, George J. Postural hypoxemia in quadriplegic patients. Neurology 1976;26:815-7.

294. McCool FD, Pichurko BM, Slutsky AS, et al. Changes in lung volume and rib cage configuration with abdominal binding in quadriplegia. J App/ Physiol 1986;60:1198-202.

295. Goldman JM, Rose LS, Williams SJ, et al. Effect of abdominal binders on breathing in tetraplegic patients. Thorax 1986:41:940-5.

296. Bodin P, Fagevik OM, Bake B, et al. Effects of abdominal binding on breathing patterns during breathing exercises in persons with tetraplegia. Spinal Cord 2005:43:117-22.

297. Jaeger RJ, Turba RM, Yarkony GM, et al. Cough in spinal cord injured patients: comparison of three methods to produce cough. Arch Phys Med Rehabil 1993:74:1358-61.

298. Seixas P, Abreu PJ, Goncalves MR. Comparison of two manually assisted coughing techniques in patients with high spinal cord injury. Eur Respir $\mathrm{J}$ 2006:28:731s.

299. Brownlee S, Williams SJ. Physiotherapy in the respiratory care of patients with high spinal injury. Physiotherapy 1987;73:148-52.

300. Bach JR. Noninvasive alternatives to tracheostomy for managing respiratory muscle dysfunction in spinal cord injury. Top Spinal Cord Inj Rehabil 1997;2:49-58.

301. Massery M. Respiratory rehabilitation secondary to neurological deficits: treatment techniques. In: Frownfelter DL, ed. Chest physical therapy and pulmonary rehabilitation. An interdisciplinary approach. St Louis MO: Mosby, 1987:529-62.

302. Bach JR. New approaches in the rehabilitation of the traumatic high leve quadriplegic. Am J Phys Med Rehabil 1991;70:13-9.

303. Bach JR, Alba AS. Noninvasive options for ventilatory support of the traumatic high level quadriplegic patient. Chest 1990;98:613-9.

304. Zupan A, Savrin R, Erjavec T, et al. Effects of respiratory muscle training and electrical stimulation of abdominal muscles on respiratory capabilities in tetraplegic patients. Spinal Cord 1997:35:540-5.

305. Stanic U, Kandare F, Jaeger R, et al. Functional electrical stimulation of abdominal muscles to augment tidal volume in spinal cord injury. IEEE Trans Rehabil Eng 2000:8:30-4.

306. Linder SH. Functional electrical stimulation to enhance cough in quadriplegia. Chest 1993:103:166-9.

307. Cheng PT, Chen CL, Wang CM, et al. Effect of neuromuscular electrical stimulation on cough capacity and pulmonary function in patients with acute cervical cord injury. $J$ Rehabil Med 2006:38:32-6.

308. Langbein WE, Maloney C, Kandare F, et al. Pulmonary function testing in spinal cord injury: effects of abdominal muscle stimulation. J Rehabil Res Dev 2001:38:591-7. 
309. Wien MF, Garshick E, Tun CG, et al. Breathlessness and exercise in spinal cord injury. J Spinal Cord Med 1999;22:297-302.

310. Bodin P, Kreuter M, Bake B, et al. Breathing patterns during breathing exercises in persons with tetraplegia. Spinal Cord 2003:41:290-5.

311. Stiller K, Huff N. Respiratory muscle training for tetraplegic patients: a literature review. Aust J Physiother 1999;45:291-9.

312. Brooks D, O'Brien K, Geddes EL, et al. Is inspiratory muscle training effective for individuals with cervical spinal cord injury? A qualitative systematic review. Clin Rehabil 2005:19:237-46.

313. Silva AC, Neder JA, Chiurciu MV, et al. Effect of aerobic training on ventilatory muscle endurance of spinal cord injured men. Spinal Cord 1998;36:240-5.

314. Biering-Sorensen $\mathbf{F}$, Lehmann KJ, Schmidt A, et al. Effect of respiratory training with a mouth-nose-mask in tetraplegics. Paraplegia 1991;29:113-9.

315. Cruzado $\mathbf{D}$, Jones $\mathrm{MD}$, Segebart $\mathrm{S}$, et al. Resistive inspiratory muscle training improves inspiratory muscle strength in subjects with cervical spinal cord injury. Neurol Rep 2002;26:3-7.

316. Wang TG, Wang YH, Tang FT, et al. Resistive inspiratory muscle training in sleepdisordered breathing of traumatic tetraplegia. Arch Phys Med Rehabil 2002;83:491-6.

317. Loveridge $\mathbf{B}$, Badour $\mathrm{M}$, Dubo $\mathrm{H}$. Ventilatory muscle endurance training in quadriplegia: effects on breathing pattern. Paraplegia 1989;27:329-39.

318. Gouden P. Progressive resistive loading on accessory expiratory muscles in tetraplegia. Fisioterapie 1990;46:4-16.

319. Liaw MY, Lin MC, Cheng PT, et al. Resistive inspiratory muscle training: its effectiveness in patients with acute complete cervical cord injury. Arch Phys Med Rehabil 2000;81:752-6.

320. Estenne M, Knoop C, Vanvaerenbergh J, et al. The effect of pectoralis muscle training in tetraplegic subjects. Am Rev Respir Dis 1989;139:1218-22.

321. Ehrlich M, Manns PJ, Poulin C. Respiratory training for a person with C3-C4 tetraplegia. Aust J Physiother 1999:45:301-7.

322. Rutchik A, Weissman AR, Almenoff PL, et al. Resistive inspiratory muscle training in subjects with chronic cervical spinal cord injury. Arch Phys Med Rehabil 1998:79:293-7.

323. Eagle M, Chatwin M. Respiratory muscle training in children and adults with neuromuscular disease. Cochrane Database Syst Rev 2006;(3):CD006155.

324. Schramm CM. Current concepts of respiratory complications of neuromuscular disease in children. Curr Opin Pediatr 2000;12:203-7.

325. Kang SW, Bach JR. Maximum insufflation capacity: vital capacity and cough flows in neuromuscular disease. Am J Phys Med Rehabil 2000;79:222-7.

326. American Thoracic Society. Respiratory care of the patient with Duchenne muscular dystrophy: ATS consensus statement. Am J Respir Crit Care Med 2004; 170:456-65.

327. Perrin C, Unterborn JN, D'Ambrosio C, et al. Pulmonary complications of chronic neuromuscular diseases and their management. Muscle Nerve 2004;29:5-27.

328. Gay PC, Edmonds LC. Severe hypercapnia after low-flow oxygen therapy in patients with neuromuscular disease and diaphragmatic dysfunction. Mayo Clin Proc 1995; 70:327-30.

329. Bach JR, Saporito LR. Criteria for extubation and tracheostomy tube removal for patients with ventilatory failure. A different approach to weaning. Chest 1996;110:1566-71.

330. Hanayama K, Ishikawa Y, Bach JR. Amyotrophic lateral sclerosis. Successful treatment of mucous plugging by mechanical insufflation-exsufflation. Am J Phys Med Rehabil 1997;76:338-9.

331. Bach JR, Ishikawa Y, Kim H. Prevention of pulmonary morbidity for patients with Duchenne muscular dystrophy. Chest 1997;112:1024-8.

332. Kang SW, Bach JR. Maximum insufflation capacity. Chest 2000;118:61-5.

333. Sivasothy $\mathbf{P}$, Brown $\mathrm{L}$, Smith $\mathrm{IE}$, et al. Effect of manually assisted cough and mechanical insufflation on cough flow of normal subjects, patients with chronic obstructive pulmonary disease (COPD), and patients with respiratory muscle weakness. Thorax 2001:56:438-44.

334. Gomez-Merino E, Bach JR. Duchenne muscular dystrophy: prolongation of life by noninvasive ventilation and mechanically assisted coughing. Am J Phys Med Rehabil 2002:81:411-5

335. Chatwin M, Ross E, Hart N, et al. Cough augmentation with mechanical insufflation/exsufflation in patients with neuromuscular weakness. Eur Respir $J$ 2003;21:502-8

336. Sancho J, Servera E, Diaz J, et al. Efficacy of mechanical insufflation-exsufflation in medically stable patients with amyotrophic lateral sclerosis. Chest 2004:125:1400-5.

337. Winck JC, Goncalves MR, Lourenco C, et al. Effects of mechanical insufflationexsufflation on respiratory parameters for patients with chronic airway secretion encumbrance. Chest 2004;126:774-80.

338. Bach JR. Amyotrophic lateral sclerosis: prolongation of life by noninvasive respiratory aids. Chest 2002;122:92-8.

339. Mustfa N, Aiello M, Lyall RA, et al. Cough augmentation in amyotrophic lateral sclerosis. Neurology 2003;61:1285-7.

340. Bach JR. Amyotrophic lateral sclerosis. Communication status and survival with ventilatory support. Am J Phys Med Rehabil 1993;72:343-9.

341. Poponick JM, Jacobs I, Supinski G, et al. Effect of upper respiratory tract infection in patients with neuromuscular disease. Am J Respir Crit Care Med 1997:156:659-64.

342. Anderson JL, Hasney KM, Beaumont NE. Systematic review of techniques to enhance peak cough flow and maintain vital capacity in neuromuscular disease: the case for mechanical insufflation-exsufflation. Phys Ther Rev 2005;10:25-33.

343. Trebbia G, Lacombe M, Fermanian C, et al. Cough determinants in patients with neuromuscular disease. Respir Physiol Neurobiol 2005:146:291-300.
344. Bach JR. Amyotrophic lateral sclerosis: predictors for prolongation of life by noninvasive respiratory aids. Arch Phys Med Rehabil 1995;76:828-32.

345. Kelleher WH, Parida RK. Glossopharyngeal breathing; its value in the respiratory muscle paralysis of poliomyelitis. Br Med J 1957:2:740-3.

346. Dail CW, Affeldt JE, Collier CR. Clinical aspects of glossopharyngeal breathing; report of use by one hundred postpoliomyelitic patients. J Am Med Assoc 1955;158:445-9.

347. Pool GM, van Weerden GJ. Experiences with frog breathing tetraplegic polio victims as telephone operators. Paraplegia 1973;11:253-25.

348. Bianchi C, Grandi M, Felisari G. Efficacy of glossopharyngeal breathing for a ventilator-dependent, high-level tetraplegic patient after cervical cord tumor resection and tracheotomy. Am J Phys Med Rehabil 2004:83:216-9.

349. James WS III, Minh VD, Minteer MA, et al. Cervical accessory respiratory muscle function in a patient with a high cervical cord lesion. Chest 1977;71:59-64.

350. Clough P. Glossopharyngeal breathing: its application with a traumatic quadriplegic patient. Arch Phys Med Rehabil 1983;64:384-5.

351. Metcalf VA. Vital capacity and glossopharyngeal breathing in traumatic quadriplegia. Phys Ther 1966;46:835-8.

352. Montero JC, Feldman DJ, Montero D. Effects of glossopharyngeal breathing on respiratory function after cervical cord transection. Arch Phys Med Rehabil 1967:48:650-3.

353. Warren VC. Glossopharyngeal and neck accessory muscle breathing in a young adult with C2 complete tetraplegia resulting in ventilator dependency. Phys Ther 2002:82:590-600

354. Benditt J0. Management of pulmonary complications in neuromuscular disease. Phys Med Rehabil Clin N Am 1998:9:167-85.

355. Johnson EW, Reynolds HT, Stauch D. Duchenne muscular dystrophy: a case with prolonged survival. Arch Phys Med Rehabil 1985;66:260-1.

356. Moloney E, Doyle S, Kinahan J, et al. A case of frog breathing. Ir Med J 2002:95:81-2

357. Mazza FG, DiMarco AF, Altose MD, et al. The flow-volume loop during glossopharyngeal breathing. Chest 1984;85:638-40.

358. Bach JR. Mechanical insufflation-exsufflation. Comparison of peak expiratory flows with manually assisted and unassisted coughing techniques. Chest 1993;104:1553-62

359. Bach JR. Respiratory muscle aids for the presenting of pulmonary morbidity and mortality. Semin Neurol 1995;15:72-83.

360. Gilmartin ME. Body ventilators. Equipment and techniques. Respir Care Clin N Am 1996:2:195-222.

361. Birnkrant DJ, Pope JF, Lewarski J, et al. Persistent pulmonary consolidation treated with intrapulmonary percussive ventilation: a preliminary report. Pediat Pulmonol 1996:21:246-9.

362. Toussaint $\mathbf{M}, \mathrm{De} \mathbf{W H}$, Steens $\mathbf{M}$, et al. Effect of intrapulmonary percussive ventilation on mucus clearance in Duchenne muscular dystrophy patients: a preliminary report. Respir Care 2003;48:940-7.

363. Estrup C, Lyager S, Noeraa N, et al. Effect of respiratory muscle training in patients with neuromuscular diseases and in normals. Respiration 1986;50:36-43.

364. Olgiati R, Girr A, Hugi L, et al. Respiratory muscle training in multiple sclerosis: a pilot study. Schweiz Arch Neurol Psychiatr 1989;140:46-50.

365. Weiner $\mathbf{P}$, Gross $D$, Meiner Z, et al. Respiratory muscle training in patients with moderate to severe myasthenia gravis. Can J Neurol Sci 1998;25:236-41.

366. Klefbeck B, Lagerstrand L, Mattsson E. Inspiratory muscle training in patients with prior polio who use part-time assisted ventilation. Arch Phys Med Rehabil 2000:81:1065-71.

367. DiMarco AF, Kelling JS, DiMarco MS, et al. The effects of inspiratory resistive training on respiratory muscle function in patients with muscular dystrophy. Muscle Nerve 1985;8:284-90.

368. Martin AJ, Stern L, Yeates J, et al. Respiratory muscle training in Duchenne muscular dystrophy. Dev Med Child Neurol 1986;28:314-8.

369. Gross $\mathbf{D}$, Meiner $Z$. The effect of ventilatory muscle training on respiratory function and capacity in ambulatory and bed-ridden patients with neuromuscular disease. Monaldi Arch Chest Dis 1993:48:322-6.

370. Wanke T, Toifl K, Merkle M, et al. Inspiratory muscle training in patients with Duchenne muscular dystrophy. Chest 1994;105:475-82.

371. Winkler G, Zifko U, Nader A, et al. Dose-dependent effects of inspiratory muscle training in neuromuscular disorders. Muscle Nerve 2000;23:1257-60.

372. Koessler W, Wanke T, Winkler G, et al. 2 Years' experience with inspiratory muscle training in patients with neuromuscular disorders. Chest 2001;120:765-69.

373. Stamler JS, Meissner G. Physiology of nitric oxide in skeletal muscle. Physiol Rev 2001:81:209-37.

374. Sander M, Chavoshan B, Harris SA, et al. Functional muscle ischemia in neuronal nitric oxide synthase-deficient skeletal muscle of children with Duchenne muscular dystrophy. Proc Natl Acad Sci USA 2000:97:13818-23.

375. Cystic Fibrosis Trust, Clinical Standards and Accreditation Group. Standards for the clinical care of children and adults with cystic fibrosis in the UK. Cystic Fibrosis Trust 2001

376. The Use of Non-Invasive Ventilation in the management of patients with chronic obstructive pulmonary disease admitted to hospital with acute type II respiratory failure (with particular reference to Bilevel positive pressure ventilation). http://brit-thoracic.org.uk/Portals/0/Clinical\%20Information/NIV/ Guidelines/NIVinCOPDFullguidelineFINAL.pdf (accessed March 2009).

377. Ram FS, Wellington SR, Barnes NC. Inspiratory muscle training for asthma. Cochrane Database Syst Rev 2003:(3):CD003792. 


\section{APPENDIX A GLOSSARY OF PHYSIOTHERAPY TERMS}

Terms used in physiotherapy for respiratory conditions. Common physiological terms

and expressions, defined in respiratory physiology books, are not included.

Term
Active cycle of breathing techniques (ACBT)
Air flow
Air flow velocity
Airway clearance technique (ACT)
Assisted autogenic drainage (AAD)
Autogenic drainage (AD)
Bilevel positive airway pressure (BiPAP)
The “Bird”
Blow-as-you-go!
Bottle-blowing/bubble PEP
Breathing control (BC)
Breathing exercises

Buteyko breathing technique (BBT)

Chest clapping/percussion

Chest compression

Chest mobility exercise(s)

Chest physiotherapy

\section{Chest shaking/vibrations}

Continuous positive airway pressure (CPAP)

Cough technique

Cough control

Delta rollator frame

Diaphragmatic breathing

Directed coughing

Energy conservation (EC)

Expiratory muscle training (EMT)

Expiratory resistance breathing (ERB)

Forced expiration technique (FET)

Forward lean sitting (FLS)

Glossopharyngeal breathing (GPB) (frog breathing)

Gutter rollator frame

High-frequency chest wall oscillator/oscillation (HFCWO)

High positive expiratory pressure (Hi-PEP)

Huff, huffing

Humidification

Hypertonic saline (HTS)

Inhalation device

Inhalation therapy

Inspiratory muscle training (IMT)

\section{Definition}

An airway clearance technique. A cycle of the techniques of breathing control (BC), thoracic expansion exercises (TEE) and the forced expiration technique (FET).

Expressed volume/time ( $\mathrm{l} / \mathrm{min})$.

Expressed distance/time ( $\mathrm{m} / \mathrm{min})$, speed.

An airway clearance strategy (with or without a device) used to support mucus clearance by loosening, mobilising, transporting and evacuating airway mucus.

Autogenic drainage (AD) carried out with assistance in infants, toddlers or individuals unable to follow instructions or to participate actively.

An airway clearance technique utilising optimal expiratory flow rate at different lung volume levels.

Assisted ventilation with independent settings for positive inspiratory and expiratory pressures.

See Intermittent positive pressure breathing.

A term to help remind the patient to exhale on effort, stretching and bending to improve respiratory mechanics during activity.

"Positive expiratory pressure" generated by blowing through a narrow tube into water.

Normal tidal breathing encouraging relaxation of the upper chest and shoulders.

Exercises designed to alter breathing for a particular purpose-for example, increasing lung volumes, decreasing lung volumes, airway clearance.

A compilation of "reduced breathing" exercises and other strategies for control of asthma symptoms; it is a more intensive and broader therapy than the conventional physiotherapy technique but with similar results.

Rhythmical percussion (tapping) of the chest wall using either the hand(s) with a flexion/extension action of the wrist(s) or a mechanical device, with the aim of loosening secretions.

Firm manual or mechanical compression of the chest during expiration in the direction of the normal expiratory movement of the ribs-that is, down and in to enhance "air flow" or "cough peak flow". Commonly combined with "chest shaking/ vibrations" to enhance airway clearance. Can be used as a "manually assisted cough" technique.

Physical flexibility exercises to maintain or increase the mobility of the chest wall.

Historical ambiguous term used to define airway clearance therapy. Commonly in the USA refers to "postural drainage" with "percussion", with or without "chest compression".

Shaking or vibrating the chest wall during expiration, in the direction of rib movement; usually combined with "chest compression"

Assisted ventilation with the same positive pressure setting during the whole breathing cycle.

Using cough in a controlled way, at specific lung volumes, to check for and/or assist the removal of bronchial secretions.

Being able to control the cough, to prevent unproductive paroxysms of coughing or coughing attacks.

A triangular "rollator frame"; highly manoeuvrable and may have a carrying bag attached for ambulatory oxygen.

Breathing using abdominal movement; reducing the degree of chest wall movement as much as possible. Not advocated in patients with hyperinflation. Commonly used in complementary therapies.

Coughing under instruction; direction given on technique, timing, frequency and duration

A method of performing tasks and activities to utilise breathing, pacing and positional strategies to reduce the work of a task/ activity.

Breathing out against a resistance as a means of enhancing strength or endurance of the expiratory muscles. Can be against a fixed load or via a threshold load resistor.

Breathing out against a resistance. Type and size of resistance chosen dependent on physiological strategy, aims and individual needs. May be used for, for example, airway clearance or "respiratory muscle training".

Huffs/forced expirations interspersed with periods of "breathing control".

Seated, leaning forwards, relaxed; supporting the elbows on either a table or own knees. Enhances respiratory muscle function by loading the diaphragm and by passive fixation of the shoulder girdle. Commonly used where there is hyperinflation of the lungs and increased FRC, as in COPD.

A method of breathing using the tongue and soft palate (as a frog does) to push air into the lungs for enhancing inspiration in an individual with weak inspiratory muscles.

A "rollator frame" with a shoulder height support for the forearms; used for severely breathless patients to allow mobility which otherwise is very difficult.

A device/technique to oscillate the chest wall externally by means of a pneumatic jacket to aid loosening of secretions.

An airway clearance technique combining "positive expiratory pressure" with forced expirations against the resistor. A huff is a forced expiration with an open glottis; when performed from a large lung volume moves central secretions; from a mid lung volume moves peripheral secretions.

Adding moisture to inhaled air or oxygen to prevent drying of mucosa and/or secretions and to improve gas exchange; may be sterile water or normal saline via nebuliser chambers, both large and small volume and via a heated water bath.

A solution of (commonly $7 \%$ in the UK but may be $9 \%$ ) saline to increase fluid flux from the airways into the mucus to improve secretion clearance; usually advocated preairway clearance

A device through which aerosolised or powdered drugs can be inhaled.

Delivery of aerosolised or powdered drugs to the airways through inhalation.

Breathing in against a resistance as a means of enhancing strength or endurance of the inspiratory muscles. Can be against a fixed load or via a threshold load resistor. 
Table Continued

Term Definition

Intermittent positive pressure breathing (IPPB)

The original form of "NIV"; pressure cycled, powered by compressed gas with an integral nebuliser; flow rate, sensitivity and pressure are adjustable. Mouthpiece is the most commonly used interface but may be delivered via a port-free mask (without a fixed leak), as closed circuit system.

Intrapulmonary percussive ventilation (IPV)

A device to oscillate/percuss the chest internally to aid loosening of secretions, by means of high-frequency bursts of gas Powered by compressed gas and can be used to deliver nebulised drugs during treatment.

Manually assisted cough (MAC)

Firm compression of the chest wall or abdomen during expiration to enhance a weak cough in an individual with weak/ paralysed expiratory muscles. Often combined with a "maximum insufflation capacity" technique.

Manual techniques

Manual therapy

The collective term for chest compression, chest shaking and chest wall vibrations; see individual techniques.

The treatment of joints and muscles by specific mobilisation, manipulation and stretching.

Maximum insufflation capacity (MIC)

Enhancing inspiration prior to a cough in an individual with weak inspiratory muscles; via "chest compression", "NIV", "IPPB", a bag or "glossopharyngeal breathing". Commonly used with a "manually assisted cough".

Mechanical in-exsufflation (MI-E)

A device to provide both positive and negative pressure in alternating cycles to enhance "MIC" and/or "cough peak flow" to enhance airway clearance. Can be combined with other techniques, most commonly a "manually assisted cough" technique. The adaptation of "postural drainage" - that is to eliminate head-down positions.

Modified postural drainage

Mucociliary clearance

The physiological movement of airway mucus by the mucociliary transport system, in a cephalad direction (towards the mouth).

Nebuliser

Nebuliser system

Nijmegen Questionnaire

Non-invasive ventilation (NIV)

Oscillating positive expiratory pressure (OscPEP)

Paced breathing

Peak cough flow (PCF)

Percussion

Physical activity

Physical exercise

Physical training

Positioning

Positive expiratory pressure (PEP)

Postural drainage (PD)

Pursed lips breathing (PLB)

Reduced breathing

Respiratory muscle training (RMT)

Reverse Trendelenburg position

Rib-springing

Rollator frame

Self-percussion

Shaking

Slow, deep breathing

Thoracic expansion exercise (TEE) (deep breathing)

Vibrations

Visual analogue scale

Trendelenburg position

A device that aerosolises a liquid

Equipment comprising an energy source and a nebuliser. These function as a unit.

A self-complete tool for measuring symptoms of hyperventilation.

Assisted ventilation applied non-invasively via a mask or mouthpiece for spontaneously breathing patients.

An airway clearance technique which utilises the effects of oscillating "positive expiratory pressure" and oscillating flow, combined with cough or "FET".

Breathing to a rhythm — for example, in time with walking or stairs, to help maintain control of breathing and thereby reduce dyspnoea.

The peak flow an individual can generate with a cough through a peak flow meter. Used to gauge cough effectiveness in individuals with respiratory muscle weakness.

See "Chest clapping/percussion".

Used to influence breathing pattern, ventilation and ventilation distribution, and to preserve physical function and flexibility; sports, activities of daily living (ADL), etc.

Targeted exercise(s) to preserve/improve a specific physical function.

A prescribed programme of physical exercise to improve/maintain exercise capacity and endurance, mobility, muscle strength and posture.

The use of different body positions to maintain joint and/or soft tissue length; improve the mechanics of breathing; utilise the effects of gravity to facilitate drainage of bronchial secretions; stimulate both skeletal and smooth muscle postural tone; and increase regional ventilation and/or perfusion.

An airway clearance technique which utilises the effects of tidal volume breathing towards an expiratory resistance, combined with FET or cough.

The use of gravity for drainage of secretions guided by bronchial anatomy.

The generation of a positive pressure within the airways by expiration against partially closed lips, as in whistling.

A technique using smaller than usual tidal volume and/or lower respiratory rate and increasing relaxation; used for patients with hyperventilation syndrome or stable asthma for control of symptoms; also a key part of the "Buteyko breathing technique".

Breathing in or out against a resistance as a means of enhancing strength or endurance of the inspiratory or expiratory muscles, respectively. Can be against a fixed load or via a threshold load resistor.

Supine position without flexing or extending, with the head higher than the feet.

A term used to describe a form of "chest compression" with overpressure at the end of expiration to enhance inspiration via stretch reflexes in individuals with weak inspiratory muscles or unable to cooperate.

A walking frame with wheels back and front for ease of use by breathless patients as it allows fixation of the shoulder girdle.

Performing "Chest clapping/percussion" independently.

See "Chest shaking/vibrations".

A technique used during exertion/exercise to help maintain control of breathing and reduce dyspnoea in tachypnoeic patients.

Deep inspiration towards inspiratory capacity; the independent means of achieving "MIC".

See "Chest shaking/vibrations".

An arbritrary linear score of $10 \mathrm{~cm}$ to represent the range of possible symptom perception.

Supine position with the feet higher than the head.

Adapted with permission from the International Physiotherapy Group for Cystic Fibrosis (IPG/CF) 2007 www.cfww.org/lPG-CF/index.asp 


\section{APPENDIX B ALGORITHM FOR THE MANAGEMENT OF PATIENTS WITH NEUROMUSCULAR WEAKNESS}

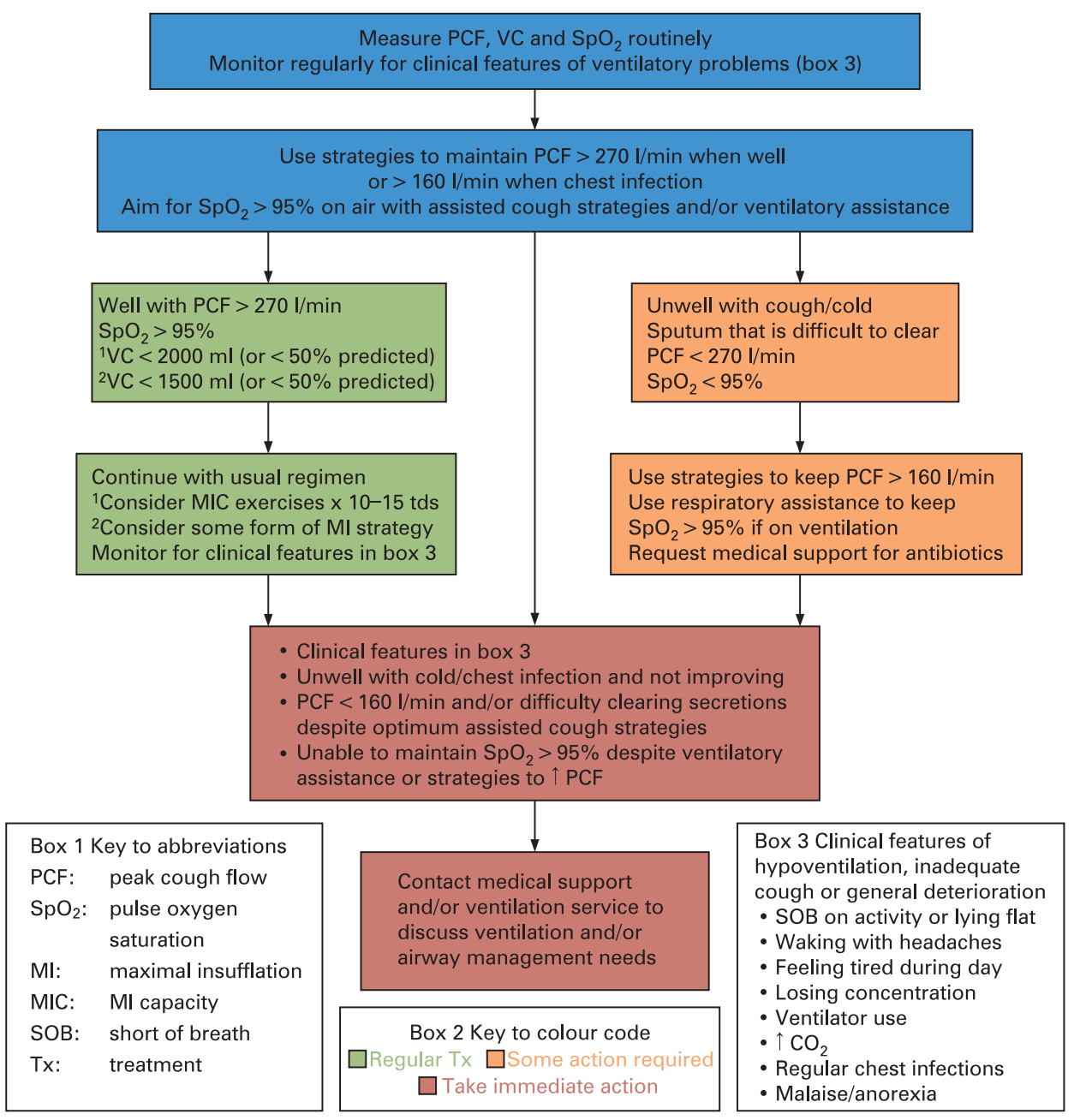

\section{APPENDIX C INSTRUCTIONS FOR PERFORMING COUGH PEAK FLOW MEASUREMENTS IN PATIENTS WITH NEUROMUSCULAR WEAKNESS \\ Equipment \\ - Peak flow meter \\ - Mouthpiece \\ - Full face mask with a good seal for a patient with weak facial muscles}

\section{Method}

- Select a position of comfort for your patient

\section{Performing unassisted peak cough flow}

- Ask the patient to take a maximal deep breath in

- Ask them to seal their lips tightly round the tube or apply the mask firmly to the face

- Ask them to COUGH as hard as possible into the peak flow mouthpiece or mask
Performing assisted peak cough flow

Patients who are able to breath stack (without bulbar weakness)

Aim for maximal insufflation capacity (MIC) by breath stacking via either:

- A volume preset ventilator

- Glossopharyngeal breathing

- A manual resuscitator bag

Patients who are unable to breath stack due to bulbar insufficiency Aim to achieve inspiration to MIC in a single breath by insufflating via either:

- A volume or pressure preset ventilator

- A manual resuscitator bag

- A mechanical insufflator-exsufflator

When the patient has achieved maximal insufflation capacity

- Ask them to seal their lips tightly round the tube or apply the mask firmly to the face

- Ask them to COUGH as hard as possible into the peak flow mouthpiece or mask with manual or mechanical assistance as required 